quatrième série-tome $44 \quad$ fascicule 6 novembre-décembre 2011

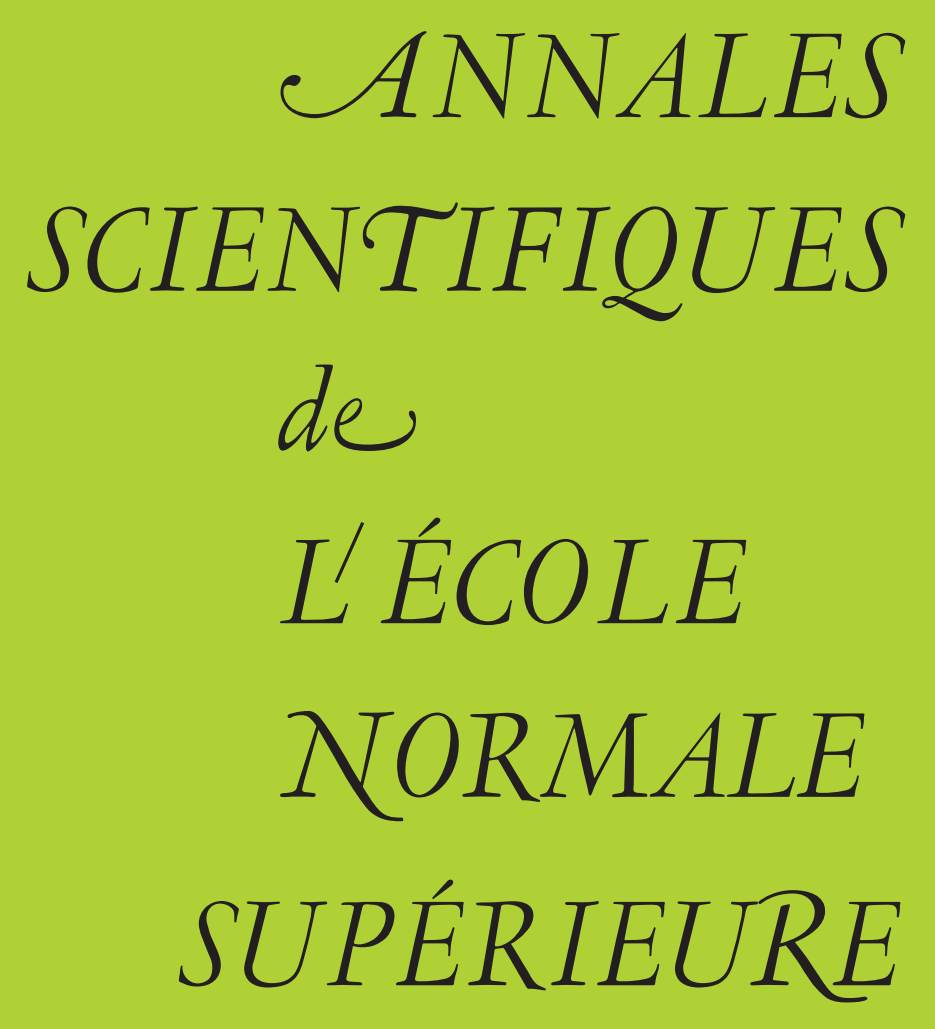

Laurent FARGUES

La filtration canonique des points de torsion des groupes $p$-divisibles 
Ann. Scient. Éc. Norm. Sup.

$4^{\text {e }}$ série, t. 44, 2011, p. 905 à 961

\title{
LA FILTRATION CANONIQUE DES POINTS DE TORSION DES GROUPES $p$-DIVISIBLES
}

\author{
PAR LAURENT FARGUES \\ AVEC LA COLLABORATION DE YICHAO TIAN
}

RÉsumÉ. - Étant donnés un entier $n \geq 1$ et un groupe de Barsotti-Tate tronqué d'échelon $n$ et de dimension $d$ sur un anneau de valuation d'inégales caractéristiques, nous donnons une borne explicite sur son invariant de Hasse qui implique que sa filtration de Harder-Narasimhan possède un sousgroupe libre de rang $d$. Lorsque $n=1$ nous redémontrons également le théorème d'Abbes-Mokrane ([1]) et de Tian ([36]) par des méthodes locales. On applique cela aux familles $p$-adiques de tels objets et en particulier à certaines variétés de Shimura de type PEL afin de montrer l'existence de familles compatibles de sections de certaines correspondances de Hecke sur des voisinages tubulaires explicites du lieu ordinaire.

Abstract. - Given an integer $n \geq 1$ and a truncated Barsotti-Tate group of level $n$ and dimension $d$ over an unequal characteristic valuation ring, we give an explicit bound on its Hasse invariant so that its Harder-Narasimhan filtration has a break which is free of rank $d$. When $n=1$ we also give a local proof of the Abbes-Mokrane ([1]) and Tian ([36]) theorem. We apply this to $p$-adic families of such objects and, in particular, we prove the existence of compatible families of sections of some Hecke correspondences on explicit tubular neighborhoods of the ordinary locus in some PEL type Shimura varieties.

\section{Introduction}

1.1. - Soit $p$ un nombre premier. Soient $K$ une extension valuée complète de $\mathbb{Q}_{p}$ de valuation discrète et $A$ un schéma abélien de dimension $g \operatorname{sur} \theta_{K}$. Si $A$ a réduction ordinaire sur le corps résiduel de $K$ et $n \geq 1$ est un nombre entier, les points de $p^{n}$-torsion de $A$ sont munis d'une filtration canonique

$$
0 \longrightarrow A\left[p^{n}\right]^{0} \longrightarrow A\left[p^{n}\right] \longrightarrow A\left[p^{n}\right]^{\text {ét }} \longrightarrow 0
$$

où $A\left[p^{n}\right]^{0}$ est un schéma en groupes de type multiplicatif d'ordre $p^{n g}$ et $A\left[p^{n}\right]^{\text {ét }}$ est étale du même ordre. Soit $\phi_{\text {ord }}$ le lieu formé des points à bonne réduction ordinaire dans l'espace analytique rigide $p$-adique $\&$ associé aux variétés de Siegel de niveau premier à $p$. C'est un ouvert admissible au sens de la géométrie rigide que l'on peut voir comme le tube au-dessus 
de l'ouvert d'ordinarité de la réduction modulo $p$ des modèles entiers canoniques de ces variétés. Soit $\phi_{\mathfrak{P}_{n}} \longrightarrow \&$ le revêtement étale fini associé au sous-groupe de congruence

$$
\mathfrak{P}_{n}=\left\{x \in \mathrm{GSp}_{2 g}\left(\mathbb{Z}_{p}\right) \mid x \equiv\left(\begin{array}{c}
* * \\
0 *
\end{array}\right) \bmod p^{n}\right\},
$$

les blocs de la matrice précédente étant de taille $g \times g$. Sur le lieu ordinaire, les filtrations précédentes se mettent en famille et fournissent une section du revêtement $\phi_{\mathfrak{P}_{n}} \rightarrow \varnothing$. Lorsque $n$ varie, ces filtrations vérifient certaines relations de compatibilité.

Sur la réduction modulo $p$ des variétés de Siegel, il y a une forme automorphe algébrique de poids $p-1$. Sa valuation définit une fonction « invariant de Hasse»

$$
\mathrm{Ha}: \varnothing \longrightarrow[0,1] \text {. }
$$

De plus, le lieu d'ordinarité de $\&$ est exactement le lieu $\mathrm{Ha}^{-1}(\{0\})$. Se pose alors la question de savoir si l'on peut étendre pour un $n$ donné la section canonique précédente sur un voisinage tubulaire $\mathrm{Ha}^{-1}\left(\left[0, \epsilon_{n}[)\right.\right.$ de $\phi_{\text {ord }}$ pour un $\epsilon_{n} \in \mathbb{Q}_{>0}$ que l'on aimerait pouvoir contrôler. La question précédente s'étend en un problème plus général concernant les groupes de Barsotti-Tate tronqués (pour l'étude du même problème dans le cas des variétés de Shimura autres que les variétés de Siegel, le cas des points de torsion des schémas abéliens est insuffisant).

Le cas des courbes elliptiques a été complètement résolu par Katz ([25]) et Lubin ([30]). Dans l'article [1] Abbes et Mokrane ont résolu le cas des variétés abéliennes de dimension générale lorsque $n=1$, c'est-à-dire le cas des points de $p$-torsion. Ils utilisent pour cela la description donnée par Bloch et Kato des cycles évanescents $p$-adiques sur les variétés projectives lisses ayant bonne réduction, couplée à la théorie de la ramification développée par Abbes et Saito dans [2]. Dans l'article [36], Tian a étendu le résultat d'Abbes-Mokrane au cas des groupes de Barsotti-Tate tronqués d'échelon 1. Il fait usage pour cela de résolutions de tels groupes par des schémas abéliens et des résultats de Bloch-Kato sur les cycles évanescents $p$-adiques associés. Dans l'article [3] Andreatta et Gasbarri ont retrouvé le résultat d'Abbes-Mokrane par d'autres méthodes globales, c'est-à-dire faisant intervenir des schémas abéliens. Conrad a montré dans [10] la surconvergence en général pour les points de $p^{n}$-torsion des schémas abéliens pour tout $n$ mais sans borne explicite. Le cas des variétés modulaires de Hilbert a été étudié en détail dans [26], [21] et [22]. Notons enfin que, dans [32], des résultats sur les sous-groupes canoniques de niveau quelconque ont été obtenus par des méthodes complètement différentes. Ces résultats concernent d'autres filtrations des schémas en groupes finis et plats que celles que nous utilisons (ces filtrations interviennent tout de même dans la section 3 où nous les appelons filtrations de ramification inférieure naïves, mais uniquement comme intermédiaires pour en étudier d'autres).

1.2. - Nous commençons tout d'abord par redémontrer le théorème d'Abbes-Mokrane et Tian par des méthodes locales ne faisant pas intervenir de schémas abéliens (cependant contrairement à Abbes et Mokrane, nous ne traitons pas dans ce texte le cas des schémas semi-abéliens). Nous précisons également le comportement de leurs filtrations vis-à-vis de la dualité et donc des polarisations. Voici le théorème démontré dans la section 6. On fixe une 
extension valuée complète $K \mid \mathbb{Q}_{p}$ pour une valuation à valeurs dans $\mathbb{R}$. On suppose de plus que $p \neq 2,3$ dans le reste de cette introduction.

ThÉORÈme (Théorème 4 point (2) et Corollaire 2). - Soit G un groupe de Barsotti-Tate tronqué d'échelon 1 , de hauteur $h$ et de dimension $d<h$ sur $\theta_{K}$. Soit $\left(G_{\mathrm{AS}}^{\lambda}\right)_{\lambda>0}$ la filtration d'Abbes-Saito de G. Supposons que son invariant de Hasse $w \in[0,1]$ soit strictement plus petit que $\frac{1}{2}$. Alors pour $\frac{w}{p-1} \leq \lambda<\frac{p}{p-1}(1-w)$ le groupe $G_{\mathrm{AS}}^{\lambda}$ est de rang $d$, indépendant de $\lambda$. Il en est de même de $G^{D}$, la filtration étant alors de rang $h-d$. Pour $\lambda$ comme précédemment, via l'accouplement $G\left(\theta_{\bar{K}}\right) \times G^{D}\left(\theta_{\bar{K}}\right) \rightarrow \mathbb{F}_{p}(1)$, on a l'égalité $\left(G^{D}\right)_{\mathrm{AS}}^{\lambda}\left(\theta_{\bar{K}}\right)^{\perp}=G_{\mathrm{AS}}^{\lambda}\left(\theta_{\bar{K}}\right)$.

La démonstration de ce théorème fait intervenir une étude fine de l'application de HodgeTate des schémas en groupes finis et plats sur $\theta_{K}$. Dans la section 6 nous démontrons d'autres résultats concernant cette application qui sont utiles dans la suite, notamment le résultat suivant.

ThÉORÈme (Théorème 4 point (3)). - Sous les hypothèses du théorème précédent la réduction du cran de rang d de la filtration de $G$ modulo les éléments de $\theta_{K}$ de valuation supérieure ou égale à $1-w$ cö̈ncide avec le noyau du morphisme de Frobenius de la réduction de $G$.

L'un des résultats-clefs pour la suite est également le théorème suivant (qui de notre avis, en dehors de l'existence des sous-groupes canoniques, est un des résultats les plus importants de cet article cf. section 9.2). Si $E$ est un schéma en groupes fini et plat sur $\theta_{K}$ on note $\operatorname{deg}(E)=\sum_{i} v\left(a_{i}\right)$ lorsque $\omega_{E} \simeq \oplus_{i} \vartheta_{K} / a_{i} \vartheta_{K}$, la valuation du «discriminant » de $E$.

ThÉorème (Théorème 4 point (1)). - Sous les hypothèses précédentes si $C \subset G$ désigne le sous-groupe canonique construit précédemment alors $\operatorname{deg}(G / C)=\mathrm{Ha}(G)$.

1.3. - Le second but de cet article est le suivant. Dans [16] l'auteur a développé une théorie des filtrations de Harder-Narasimhan des schémas en groupes finis et plats. Nous utilisons cette théorie afin de construire des sous-groupes canoniques en niveau quelconque dans la section 7 . Voici une version abrégée du théorème 6 de cette section.

ThÉORÈme. - Soit $n \geq 1$ un nombre entier. Soit $G$ un groupe de Barsotti-Tate tronqué d'échelon $n$, de hauteur $h$ et de dimension $d<h$ sur $\theta_{K}$. Soit $w \in[0,1]$ son invariant de Hasse. Supposons que

$$
w<\frac{1}{2 p^{n-1}} .
$$

La filtration de Harder-Narasimhan de G possède alors un cran $C$ tel que $C\left(\theta_{\bar{K}}\right)$ soit un $\mathbb{Z} / p^{n} \mathbb{Z}$-module libre de rang $n d$. La filtration de $G^{D}$ possède également un cran $D$ tel que $D\left(\theta_{\bar{K}}\right)$ soit libre de rang $n(h-d)$. De plus $C\left(\theta_{\bar{K}}\right)=D\left(\theta_{\bar{K}}\right)^{\perp}$.

Le théorème précédent est plus précis au sens où il comprend un résultat de compatibilité lorsque $n$ varie. Si $1 \leq k<n$, l'adhérence schématique dans $G$ de $C\left(\theta_{\bar{K}}\right)\left[p^{k}\right], C_{k}$, est un cran de la filtration de Harder-Narasimhan de $G\left[p^{k}\right]$. Soit $\epsilon_{n}=\frac{1}{2 p^{n-1}}$ la borne donnée dans le théorème précédent. On montre alors que le groupe de Barsotti-Tate tronqué d'échelon $n-k, p^{-(n-k)} C_{k} / C_{k}$ est d'invariant de Hasse strictement plus petit que $\epsilon_{n-k}$ et 
$C / C_{k}$ est le cran de sa filtration de Harder-Narasimhan libre de rang $(n-k) d$. Voici un des autres résultats que nous démontrons dans la section 7 (cf. théorème 5 et le corollaire 3 ).

THÉORÈme. - Soit $H$ un groupe $p$-divisible sur $\theta_{K}$ tel que $\mathrm{Ha}(H)<\frac{1}{2}$. Soit $C \subset H[p]$ le sous-groupe canonique du théorème précédent.

- Si $\mathrm{Ha}(H)<\frac{1}{p+1}$ alors $\mathrm{Ha}(H / C)=p \mathrm{Ha}(H)$.

- Si $\frac{1}{p+1} \leq \mathrm{Ha}(H)<\frac{1}{2}$ alors $\mathrm{Ha}(H / C) \geq 1-\mathrm{Ha}(H)$.

En particulier tout groupe p-divisible non-ordinaire sur $\emptyset_{K}$ est isogène à un groupe p-divisible d'invariant de Hasse supérieur ou égal à $\frac{1}{2}$.

Le cas des courbes elliptiques à réduction supersingulière montre que le théorème précédent est optimal.

On remarquera enfin que, dans [38], Yichao Tian démontre que le sous-groupe plat fini précédent $C$, qui est un cran de la filtration de Harder-Narasimhan de $G$ et dont on a montré qu'il est un cran de la filtration d'Abbes-Saito lorsque $n=1$, est également un cran de la filtration d'Abbes-Saito pour tout entier $n$. Cela complète donc les résultats précédents.

1.4. - On montre dans [16] que les filtrations de Harder-Narasimhan des groupes finis et plats se mettent en famille. Le théorème qui suit découle alors facilement des résultats précédents et de ceux de [16]. Nous énonçons le théorème dans le cas des variétés de Siegel mais celui-ci s'applique à d'autres cas de variétés de Shimura de type PEL (par exemple toutes celles associées à un groupe de similitudes symplectiques sur un corps totalement réel). Il est probable que les techniques de cet article s'appliquent à toutes les variétés de Shimura de type PEL (une fois défini un bon invariant de Hasse qui modifie l'invariant usuel).

On note $\mathfrak{P}_{0}=\operatorname{Gsp}_{2 g}\left(\mathbb{Z}_{p}\right)$. Si $n \geq k \geq 0$ il y a une correspondance de Hecke

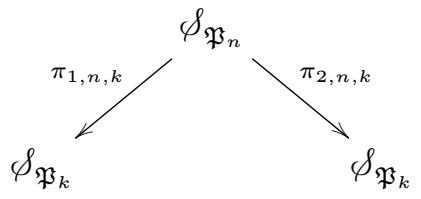

où $\pi_{1, n, k}$ est l'application d'oubli du niveau et $\pi_{2, n, k}$ associe à un couple $(A, C)$ le couple $\left(A / C\left[p^{n-k}\right], C / C\left[p^{n-k}\right]\right)$ où $A$ est une variété abélienne principalement polarisée et $C \subset A\left[p^{n}\right]$ un sous-groupe totalement isotrope maximal.

ThÉORÈme (Théorème 8). - Posons pour $n \geq 1, \epsilon_{n}=\frac{1}{2 p^{n-1}}$. Pour $k \in \mathbb{N}, \epsilon \in \mathbb{Q}, k \geq 0$ et $\epsilon>0$ on note $\left(\phi_{\mathfrak{P}_{k}}\right)_{\text {ord }}(\stackrel{\bullet}{\epsilon})$ le tube du lieu ordinaire dans $\phi_{\mathfrak{P}_{k}}$ où l'invariant de Hasse est strictement plus petit que $\epsilon$.

1. Il y a alors pour tout $n \geq 1$ une section $s_{n}$

$$
\begin{gathered}
\left(\phi_{\mathfrak{P}_{n}}\right)_{\text {ord }}\left(\stackrel{\circ}{\epsilon}_{n}\right) \\
\pi_{1, n, 0} \mid \int s_{n} \\
\phi_{\text {ord }}\left(\stackrel{\circ}{\epsilon}_{n}\right) .
\end{gathered}
$$

étendant la section canonique sur le lieu ordinaire. 
2. Posons $U_{p}=\pi_{2,1,0} \circ s_{1}: \phi_{\text {ord }}\left(\frac{i}{2}\right) \rightarrow \&$ l'opérateur «quotient par le sous-groupe canonique ». On a alors en restriction au tube $\&_{\text {ord }}\left(\frac{i}{p+1}\right)$

$$
\mathrm{Ha} \circ U_{p}=p \mathrm{Ha} \text {. }
$$

3. Lorsque $\epsilon<\frac{p-2}{p(2 p-2)}$ le morphisme induit $U_{p}: \phi_{\text {ord }}(\stackrel{\circ}{\epsilon}) \rightarrow \phi_{\text {ord }}(p \stackrel{\circ}{)}$ est étale fini et surjectif.

4. On a les relations de compatibilité suivantes

$$
\begin{aligned}
\pi_{1, n, k} \circ s_{n} & =s_{k \mid \phi_{\text {ord }}\left(\epsilon_{n}^{\circ}\right)} \\
\left.s_{k} \circ \pi_{2, n-k, 0} \circ\left(s_{n-k}\right)\right|_{\phi_{\text {ord }}\left(\epsilon_{n}^{\circ}\right)} & =\pi_{2, n, k} \circ s_{n} .
\end{aligned}
$$

1.5. - Disons quelques mots sur la stratégie utilisée pour démontrer les résultats précédents. Nous procédons à une étude fine de l'application de Hodge-Tate des groupes de BarsottiTate tronqués. Cette stratégie n'est pas nouvelle puisqu'elle apparaît déjà dans [1] et [3] sous la forme de l'étude de l'application « $d \log$ ». Dans ces articles, les auteurs caractérisent le sous-groupe canonique des points de $p$-torsion d'une variété abélienne comme étant le dual du noyau de cette application $d \log$ (cf. par exemple [1] rem. 6.1 pour une telle description conjecturale et [3] prop. 13.4 et section 13.6). C'est là que l'auteur a pris connaissance du fait que l'étude de l'application de Hodge-Tate est un outil pour étudier les sous-groupes canoniques. L'ingrédient principal que nous ajoutons est le suivant. Si $G$ est un schéma en groupes fini et plat sur $\theta_{K}$ son application de Hodge-Tate est un morphisme

$$
\alpha_{G}: G\left(\theta_{\bar{K}}\right) \longrightarrow \omega_{G^{D}} \otimes \theta_{\bar{K}} .
$$

On démontre et utilise alors le résultat suivant de théorie de Hodge $p$-adique (théo. 3) : le $\vartheta_{\bar{K}}$-module de torsion

$$
\omega_{G^{D}} \otimes \theta_{\bar{K}} / \theta_{\bar{K}} \cdot \operatorname{Im}\left(\alpha_{G}\right)
$$

est annulé par $p^{\frac{1}{p-1}}$. Ce théorème combiné à des manipulations «élémentaires ", mais astucieuses, d'algèbre linéaire et sur les schémas en groupes finis et plats fournit miraculeusement les résultats cités précédemment (le terme semble adapté car, partant de ce résultat de théorie de Hodge $p$-adique, la preuve est une succession de manipulations qui s'emboîtent de façon mystérieuse).

1.6. - Voici une description des différentes sections de l'article.

La section 2 contient des rappels et définitions sur l'invariant de Hasse et l'application de Hodge-Tate des groupes de Barsotti-Tate tronqués. On y explicite le lien entre ordinarité, invariant de Hasse et filtration de Harder-Narasimhan. Le seul résultat original est la proposition 2 reliant l'invariant de Hasse d'un $B T_{1}$ à celui de son dual de Cartier.

La section 3 contient un argument montrant l'existence d'une borne non-effective $\epsilon(n, d, h)>0$ telle que si $G$ est un $B T_{n}$ de hauteur $h$ et de dimension $d$ dont la valuation de son invariant de Hasse est plus petite que $\epsilon(n, d, h)$ alors $G$ possède un sous-groupe canonique au sens des filtrations de Harder-Narasimhan (cf. prop. 3). Pour les schémas abéliens et d'autres filtrations des schémas en groupes finis et plats, ce type de résultat noneffectif a été obtenu par Conrad dans [10]. Les résultats de cette section ne sont pas utilisés dans la suite de l'article. Cependant leur preuve est élémentaire et constitue tout de même une motivation pour les résultats effectifs qui suivent. C'est pourquoi nous l'avons inclus. 
La section 4 contient des propriétés générales des différentes filtrations des schémas en groupes finis et plats que nous utilisons dans la suite. Le point principal est la proposition 6 qui est une preuve élémentaire du théorème 1.6 de [36] reliant l'orthogonal de la filtration d'Abbes-Saito à la filtration de congruence du dual définie par Andreatta et Gasbarri dans [3].

La section 5 contient le résultat cité précédemment sur le conoyau de l'application de Hodge-Tate d'un groupe fini et plat (le théorème 3). Cette section peu paraître inutilement longue, mais nous avons préféré détailler ce résultat et l'inclure dans le corps du texte pour deux raisons. Tout d'abord il s'agit de l'ingrédient nouveau essentiel par rapport aux travaux d'Abbes-Mokrane et Andreatta-Gasbarri. De plus, ce résultat, que l'auteur a déjà utilisé dans [16] et [15], n'a semble-t-il pas été remarqué auparavant et l'auteur pense qu'il pourrait avoir des applications dans d'autres contextes. L'auteur a donc décidé de détailler ce résultat et notamment de le mettre en perspective par rapport à la presque décomposition de HodgeTate de Fontaine ([18]). Le lecteur peut très bien admettre ce résultat de théorie de Hodge $p$-adique et lire le reste de l'article.

La section 6 est le cœur de cet article. Le résultat principal en est le théorème 4. On y construit le sous-goupe canonique d'un $B T_{1}$ comme noyau de l'application de Hodge-Tate légèrement modifiée. Le point nouveau par rapport aux résultats de [1] et [3] est que l'on montre que si $C$ est le sous-groupe canonique du $B T_{1} G$ alors le degré du groupe fini et plat $G / C$ est égal à la valuation de l'invariant de Hasse de $G$ (point (1) du théorème 4 ). Ce résultat est fondamental. C'est autour de celui-ci que s'articule tout le reste de l'article et en particulier la construction du sous-groupe canonique des $B T_{n}$ pour tout entier $n$. Outre le résultat cité précédemment sur le conoyau de l'application de Hodge-Tate, la preuve du théorème 4 utilise un argument de découpage du groupe plat fini $G / C$ en une extension successive de groupes de Oort-Tate (groupes pour lesquels on peut calculer explicitement leur application de Hodge-Tate). Cela peut paraître naîf au premier abord, mais combiné à des arguments d'algèbre linéaire cela donne le résultat.

Dans la section 7 on construit le sous-groupe canonique en niveau quelconque et on démontre ses propriétés énoncées précédemment (théorème 6). Le point consiste à effectuer une récurrence à partir des sous-groupes canoniques des $B T_{1}$. Plus précisément, si l'on suppose construit le sous-groupe canonique des $B T_{n}$ et $G$ est un $B T_{n+1}$ on regarde le $B T_{n}(G / C)\left[p^{n}\right]$ où $C$ est le sous-groupe canonique de $G[p]$. Grâce au résultat énoncé précédemment sur le degré de $G[p] / C$, on dispose d'un bon contrôle sur la valuation de l'invariant de Hasse de $(G / C)\left[p^{n}\right]$ et on peut lui appliquer l'hypothèse de récurrence. Cela permet de construire un sous-groupe fini et plat $D$ de $G$ contenant $C$ et tel que $D / C$ soit le sous-groupe canonique de $(G / C)\left[p^{n}\right]$. Il s'agit ensuite de montrer que $D$ satisfait aux propriétés demandées pour le sous-groupe canonique.

La section 8 contient les applications aux variétés de Shimura énoncées précédemment. Il s'agit d'une traduction géométrique des résultats précédents.

J'aimerais exprimer mes remerciements à Yichao Tian qui dans une lettre ([37]) m'a expliqué comment aboutir aux résultats finaux de la section 7 à partir d'une version préliminaire de cet article. Je remercie également Farid Mokrane, Marc-Hubert Nicole, Vincent Pilloni, Torsten Wedhorn et Daniel Wortmann pour des remarques et des corrections.

4 ${ }^{\mathrm{e}}$ SÉRIE - TOME $44-2011$ - No 6 


\section{Notations}

Soit $p$ un nombre premier. On fixe $K \mid \mathbb{Q}_{p}$ une extension valuée complète pour une valuation $v: K \rightarrow \mathbb{R} \cup\{+\infty\}$ telle que $v(p)=1$. On ne fait aucune hypothèse sur le corps résiduel de $K$. La valuation $v$ peut être quelconque, pas forcément discrète. Si $t \in \mathbb{R}_{>0} \cap v\left(K^{\times}\right)$on notera $\mathfrak{m}_{K, t}=\left\{x \in \theta_{K} \mid v(x) \geq t\right\}$ et $\theta_{K, t}=O_{K} / \mathfrak{m}_{K, t}$. Si $M$ est un $\theta_{K}$-module on note $M_{t}:=M \otimes \theta_{K, t}$. On fixe une clôture algébrique $\bar{K}$ de $K$. On utilisera le même type de notations $\mathfrak{m}_{\bar{K}, t}, \theta_{\bar{K}, t}, M_{t}$ pour $t \in v\left(\bar{K}^{\times}\right)$et $M$ un $\vartheta_{\bar{K}}$-module.

Si $M$ est un $\theta_{K}$-module de présentation finie annulé par une puissance de $p$, on note

$$
\operatorname{deg}(M)=v\left(\operatorname{Fitt}_{0} M\right)
$$

où Fitt $_{0} M$ désigne le 0 -ième idéal de Fitting de $M$ et si $I$ est un idéal non nul de type fini de $\vartheta_{K}$ on note $v(I)=v(a)$ si $I=(a)$. En d'autres termes, si $M \simeq \bigoplus_{i \in I} \vartheta_{K} / a_{i} \vartheta_{K}$, $\operatorname{deg}(M)=\sum_{i \in I} v\left(a_{i}\right)$. On utilise le même type de notations pour un $\emptyset_{\bar{K}}$-module de présentation finie. On remarquera que cette fonction degré est additive sur les suites exactes de modules du type précédent.

Tous les schémas en groupes finis et plats que nous considérons dans cet article sont supposés commutatifs. On note $G \mapsto G^{D}$ la dualité de Cartier de tels schémas en groupes.

Nous utilisons la théorie développée dans [16]. Rappelons en particulier les notations suivantes. Si $G$ est un schéma en groupes commutatif fini et plat d'ordre une puissance de $p$ $\operatorname{sur} \theta_{K}$ on note ht $(G)=\log _{p}|G|$ et $\operatorname{deg}(G)=\operatorname{deg}\left(\omega_{G}\right)$. Enfin, si $G$ est non nul, sa pente de Harder-Narasimhan est par définition

$$
\mu(G)=\frac{\operatorname{deg} G}{\mathrm{ht} G} \in[0,1] .
$$

Rappelons également ([16]) que l'on peut associer à un tel $G$ un polygone de HarderNarasimhan que nous notons $\operatorname{HN}(G)$. Remarquons enfin le problème de terminologie suivante qui nous l'espérons ne gênera pas trop le lecteur; si $G$ est un groupe de BarsottiTate tronqué par définition sa hauteur est la hauteur au sens précédent de ses points de $p$-torsion et non de $G$ lui-même.

\section{Groupes $p$-divisibles ordinaires et invariant de Hasse}

\subsection{Quelques rappels sur les groupes finis localement libres en caractéristique $p$}

2.1.1. Groupes annulés par leur Verschiebung. - Soit $S$ un schéma tel que $p \theta_{S}=0$. Si $\mathcal{M}$ est un faisceau quasi-cohérent de $\theta_{S}$-modules on note $\underline{M}$ le faisceau fppf associé. Lorsque $\mathcal{M}$ est localement libre de rang fini $\underline{\mathcal{M}}$ est représentable par un $S$-schéma en groupes localement isomorphe pour la topologie Zariski de $S$ à une somme de copies du groupe additif $\mathbf{G}_{a}$. Pour un faisceau de groupes abéliens fppf $\mathcal{F}$ sur $S$ on note $\mathcal{F}^{(p)}=$ Frob $_{S}^{*} \mathcal{F}$ et $F: \mathcal{F} \rightarrow \mathcal{F}^{(p)}$ le morphisme de Frobenius relatif. Si $\mathcal{M}$ est un faisceau cohérent on note $\mathcal{M}^{(p)}=$ Frob $_{S}^{*} \mathcal{M}$. Les notations précédentes sont compatibles au sens où $(\underline{\mathcal{M}})^{(p)}=\underline{\mathcal{M}}^{(p)}$ que l'on notera donc sans ambiguïté $\underline{\mathcal{M}}^{(p)}$. 
Soit $\mathscr{C}$ la catégorie formée des couples $(\mathcal{M}, \psi)$ où $\mathcal{M}$ est un $\Theta_{S}$-module localement libre de rang fini et $\psi: \mathcal{M} \rightarrow \mathcal{M}^{(p)}$. D'après le théorème 7.4 de [20], il y a une équivalence de catégories

$$
\mathscr{C} \stackrel{\sim}{\longrightarrow} S \text {-schémas en groupes finis localement libres annulés par } V
$$

où $V$ désigne le Verschiebung. Cette équivalence se décrit de la façon suivante. Au couple $(\mathcal{M}, \psi)$ on associe le schéma en groupes $G$ noyau de $F-\psi$,

$$
0 \longrightarrow G \longrightarrow \underline{\mathcal{M}} \stackrel{F-\psi}{\longrightarrow} \underline{\mathcal{M}}^{(p)} \longrightarrow 0
$$

Au groupe $G$ annulé par $V$ on associe le couple $\left(\omega_{G^{D}}, \psi_{G}\right)$ où $\psi_{G}: \omega_{G^{D}} \rightarrow \omega_{\left(G^{D}\right)^{(p)}}=\omega_{G^{D}}^{(p)}$ est le morphisme induit par $F: G \rightarrow G^{(p)}$.

Si $G$ est un $S$-schéma en groupes fini localement libre, il y a un morphisme canonique

$$
\alpha_{G}: G \longrightarrow \underline{\omega}_{G^{D}}
$$

universel pour les morphismes de $G$ vers un faisceau de la forme $\underline{\mathcal{M}}$ avec $\mathcal{M}$ quasi-cohérent. Il est défini de la façon suivante :

$$
\begin{aligned}
\alpha_{G}: G=\operatorname{H} \operatorname{fom}\left(G^{D}, \mathbb{G}_{m}\right) & \longrightarrow \underline{\omega}_{G^{D}} \\
x & \longmapsto x^{*} \frac{d T}{T} .
\end{aligned}
$$

On a de plus l'égalité $\left(F-\psi_{G}\right) \circ \alpha_{G}=0$, où $\psi_{G}$ est défini comme précédemment.

Si $H$ est le groupe annulé par $V$ associé à $(\mathcal{M}, \psi)$ alors, via l'identification $\omega_{H^{D}}=\mathcal{M}$, le plongement canonique $H \hookrightarrow \underline{\mathcal{M}}$ est égal à $\alpha_{G}$. En effet, le morphisme $\alpha_{H}$ est compatible au changement de base, fonctoriel en $H$ et une section de $H$ est donnée par un morphisme $\mathbb{Z} / p \mathbb{Z} \rightarrow G$. Il suffit alors de vérifier que, pour $H=\mathbb{Z} / p \mathbb{Z}=\operatorname{ker}\left(\mathbb{G}_{a} \stackrel{F-\mathrm{Id}}{\longrightarrow} \mathbb{G}_{a}\right)$, le plongement $\mathbb{Z} / p \mathbb{Z} \subset \mathbb{G}_{a}$ est égal à $\alpha_{\mathbb{Z} / p \mathbb{Z}}$ ce qui ne pose pas de problème.

Si $H$ est un groupe annulé par $V$ comme précédemment, le foncteur $E \mapsto\left(\omega_{E^{D}}, \psi_{E}\right)$ induit la formule d'adjonction pour tout schéma en groupes fini localement libre $G$ (théorème 7.2 de [20])

$$
\operatorname{Hom}(G, H) \stackrel{\sim}{\longrightarrow} \operatorname{Hom}\left(\left(\omega_{G^{D}}, \psi_{G}\right),\left(\omega_{H^{D}}, \psi_{H}\right)\right) .
$$

L'inverse de cet isomorphisme est donné par $\alpha_{G}$ et la formule $H=\operatorname{ker}\left(F-\psi_{H}\right)$.

2.1.2. Le cas des $B T_{1}$. - On renvoie au chapitre I de [31] et à [24] pour les généralités concernant les groupes de Barsotti-Tate tronqués. Soit maintenant $G$ un groupe de Barsotti-Tate tronqué d'échelon 1 sur $S$. Le module $\omega_{G^{D}}$ est alors localement libre. Via l'équivalence de catégories précédente, le couple $\left(\omega_{G^{D}}, \psi_{G}\right)$ correspond au groupe annulé par $V, G / \operatorname{ker}(F)$. Il y a alors une suite exacte

$$
0 \longrightarrow \operatorname{ker} F \longrightarrow G \stackrel{\alpha_{G}}{\longrightarrow} \underline{\omega}_{G^{D}} \stackrel{F-\psi_{G}}{\longrightarrow} \underline{\omega}_{G^{D}}^{(p)} \longrightarrow 0 .
$$

Cette suite exacte est à la base de l'idée suivante sur laquelle se fonde la section 6 de cet article, la construction de la filtration canonique des points de $p$-torsion. Supposons que $G$ provienne par réduction modulo $p$ d'un groupe de Barsotti-Tate tronqué $G^{\prime}$ et que l'on veuille relever le noyau du Frobenius de $G$ en un sous-groupe de $G^{\prime}$. L'application $\alpha_{G}$ se relève toujours canoniquement en une application $\alpha_{G^{\prime}}$ et il est logique de s'intéresser au noyau de $\alpha_{G^{\prime}}$.

La proposition suivante sera très utile plus tard. 
Proposition 1. - Soit G un groupe de Barsotti-Tate tronqué d'échelon 1 sur un schéma annulé par $p$. Soit $C \subset G$ un sous-groupe fini localement libre. L'inclusion $C \subset \operatorname{ker} F_{G}$ est vérifiée si et seulement si l'application $\omega_{C^{D}} \longrightarrow \omega_{G^{D}}$ est nulle.

Démonstration. - Le morphisme composé $C \hookrightarrow G \rightarrow G /$ ker $F$ est donné d'après la formule d'adjonction précédente par le morphisme associé $\left(\omega_{C^{D}}, \psi_{C}\right) \rightarrow\left(\omega_{G^{D}}, \psi_{G}\right)$.

\subsection{Invariant de Hasse d'un groupe de Barsotti-Tate tronqué d'échelon 1}

2.2.1. Le cas d'un point. - Soit $G$ un groupe de Barsotti-Tate tronqué d'échelon 1, de dimension $d$ et de hauteur $h>d \operatorname{sur} \operatorname{Spec}\left(\theta_{K}\right)$. Le $\theta_{K, 1}$-module $\omega_{G^{D}}$ est libre de rang $h-d$. On a de plus l'égalité $\omega_{G^{D}}=\omega_{G^{D} \otimes \vartheta_{K, 1}}$. On note encore $\psi_{G}$ pour $\psi_{G \otimes \vartheta_{K, 1}}$. Prenant le déterminant de ce morphisme de $\vartheta_{K, 1}$-modules, on obtient un élément

$$
\widetilde{\mathrm{Ha}}(G) \in \operatorname{det}\left(\omega_{G^{D}}\right)^{\otimes(p-1)} .
$$

De façon équivalente, après avoir fixé une base de $\omega_{G^{D}}$, prenant la valuation du déterminant de $\psi_{G}$, on voit cet invariant de Hasse comme un élément

$$
\mathrm{Ha}(G) \in[0,1] .
$$

On remarquera bien sûr que si $L \mid K$ est une extension valuée complète alors

$$
\mathrm{Ha}(G)=\mathrm{Ha}\left(G \otimes_{\vartheta_{K}} \vartheta_{L}\right) .
$$

2.2.2. Le cas des familles. - Soit $K \mid \mathbb{Q}_{p}$ comme précédemment. Soit $\mathfrak{X}$ un $\operatorname{Spf}\left(\theta_{K}\right)$-schéma formel topologiquement de type fini sans $p$-torsion (un schéma formel admissible au sens de Raynaud, cf. [8]). Soit $G$ un groupe de Barsotti-Tate tronqué d'échelon 1, de dimension $d$ et de hauteur $h$ sur $\mathfrak{X}$. Le $\Theta_{\mathfrak{X}} / p \vartheta_{\mathfrak{X}}$-module $\omega_{G^{D}}$ est localement libre de rang $h-d$. On note $\psi_{G}:=\psi_{G \bmod p}$. Prenant le déterminant de $\psi_{G}$ on obtient alors un invariant de Hasse

$$
\widetilde{\operatorname{Ha}}(G) \in \Gamma\left(\mathfrak{X}, \operatorname{det}\left(\omega_{G^{D}}\right)^{\otimes(p-1)}\right) .
$$

Voyant cet invariant comme un morphisme $\widetilde{\mathrm{Ha}}(G): \oslash_{\mathfrak{X}} / p \oslash_{\mathfrak{X}} \longrightarrow \operatorname{det}\left(\omega_{G^{D}}\right)^{\otimes(p-1)}$, il induit un morphisme $\widetilde{\mathrm{Ha}}(G)^{\vee}: \operatorname{det}\left(\omega_{G^{D}}\right)^{\otimes(1-p)} \longrightarrow \Theta_{\mathfrak{X}} / p \Theta_{\mathfrak{X}}$ et donc en particulier un faisceau d'idéaux cohérent

$$
\mathrm{Ha}_{\mathfrak{I}}(G) \subset \theta_{\mathfrak{X}}
$$

contenant $p \vartheta_{\mathfrak{X}}$ et tel que $\operatorname{HaI}_{\mathfrak{I}}(G) / p \vartheta_{\mathfrak{X}}=\operatorname{Im}\left(\widetilde{\mathrm{Ha}}(G)^{\vee}\right)$ soit localement engendré par un élément.

Soit

$$
f: \mathfrak{Y} \longrightarrow \mathfrak{X}
$$

un morphisme de schémas formels du type précédent. Posons $f^{*} G:=G \times \mathfrak{X} \mathfrak{Y}$. Il y a une identification

On vérifie aussitôt les formules

$$
\omega_{\left(f^{*} G\right)^{D}}=f^{*} \omega_{G^{D}}
$$

$$
\begin{aligned}
\widetilde{\mathrm{Ha}}\left(f^{*} G\right) & =f^{*} \widetilde{\mathrm{Ha}}(G) \\
\operatorname{Ha}_{\mathfrak{I}}\left(f^{*} G\right) & =\emptyset_{\mathfrak{Y}} \cdot f^{-1} \operatorname{Ha}_{\mathfrak{I}}(G) .
\end{aligned}
$$


Soit $\pi: \widetilde{\mathfrak{X}} \longrightarrow \mathfrak{X}$ l'éclatement formel admissible de l'idéal $\mathrm{Ha}_{\mathfrak{I}}(G)$. Le diviseur exceptionnel de $\pi$ est l'idéal $\mathrm{HaI}_{\mathfrak{I}}\left(\pi^{*} G\right)$. Quitte à faire un éclatement formel admissible de la base, on peut donc toujours supposer que $\mathrm{HaI}_{\mathfrak{I}}(G)$ définit un diviseur de Cartier sur cette base.

Soit $\mathfrak{X}^{\text {rig }}$, resp. $\mathfrak{X}^{\text {an }}$, la fibre générique de $\mathfrak{X}$ comme $K$-espace rigide ([8]), resp. comme $K$-espace analytique au sens de Berkovich ([4]). Si $x \in \mathfrak{X}^{\text {an }}$ on note $\mathcal{K}(x)$ le corps résiduel associé, un corps valué complet extension de $K$. Rappelons que les points de $\mathfrak{X}^{\text {rig }}$ s'identifient aux $x \in \mathfrak{X}^{\text {an }}$ tels que $[\mathcal{K}(x): K]<+\infty$. À un point $x \in \mathfrak{X}^{\text {an }}$ est associée une spécialisation $G_{x}$ de $G$, un groupe de Barsotti-Tate tronqué sur $\bigoplus_{\mathcal{K}(x)}$. On peut donc définir l'invariant numérique

$$
\mathrm{Ha}\left(G_{x}\right) \in[0,1] .
$$

On remarquera que cet invariant ne dépend que de l'idéal $\mathrm{Ha \Im}_{\mathfrak{I}}(G)$. On vérifie facilement le lemme qui suit.

Lemme 1. - La fonction

$$
\begin{aligned}
\left|\mathfrak{X}^{\mathrm{an}}\right| & \longrightarrow[0,1] \\
x & \longmapsto \mathrm{Ha}\left(G_{x}\right)
\end{aligned}
$$

est continue. De plus si $\epsilon \in[0,1] \cap v\left(\bar{K}^{\times}\right)$le fermé

$$
\left\{x \in \mathfrak{X}^{\mathrm{an}} \mid \mathrm{Ha}\left(G_{x}\right) \leq \epsilon\right\}
$$

est un domaine analytique dans $\mathfrak{X}^{\text {an }}$ associé à un ouvert admissible quasicompact de $\mathfrak{X}^{\text {rig }}$. De même en remplaçant fermé par ouvert, $\leq$ par $<$ et en enlevant l'assertion de quasicompacité.

2.2.3. Compatibilité à la dualité. - Soit $G$ défini sur $\mathfrak{X}$ comme dans la section 2.2.2 précédente.

Proposition 2. - Supposons $0<d<h$. Il y a un isomorphisme canonique $\operatorname{det}\left(\omega_{G}\right)^{\otimes(p-1)} \simeq \operatorname{det}\left(\omega_{G^{D}}\right)^{\otimes(p-1)}$. Via cet isomorphisme $\widetilde{\mathrm{Ha}}(G)=\widetilde{\mathrm{Ha}}\left(G^{D}\right)$.

Démonstration. - Notons $X$ la réduction modulo $p$ de $\mathfrak{X}, \Sigma=\operatorname{Spec}\left(\mathbb{F}_{p}\right)$ et $\mathscr{G}$ la réduction modulo $p$ de $G$. Soit

$$
\mathcal{E}=\mathcal{E} x t_{\text {cris }}^{1}\left(\mathscr{G}, \theta_{X / \Sigma}\right)_{X}
$$

l'évaluation du cristal de Dieudonné contravariant de $\mathscr{G}$ sur l'épaississement tautologique ([7], chapitre 3), un $\vartheta_{X}$-module localement libre de rang $h$. Il y a une suite exacte de $\theta_{X}$-modules localement libres

$$
0 \longrightarrow \omega_{G} \longrightarrow \mathcal{E} \longrightarrow \omega_{G^{D}}^{\vee} \longrightarrow 0 .
$$

Soient $F: \mathscr{G} \longrightarrow \mathscr{G}^{(p)}$ le morphisme de Frobenius de $\mathscr{G}$ et $V: \mathscr{G}^{(p)} \longrightarrow \mathscr{G}$ son Verschiebung. Ils induisent des morphismes

$$
\begin{aligned}
& F^{*}: \mathcal{E}^{(p)} \longrightarrow \mathcal{E} \\
& V^{*}: \mathcal{E} \longrightarrow \mathcal{E}^{(p)}
\end{aligned}
$$

$4^{\text {e }}$ SÉRIE - TOME $44-2011-$ NNo $^{\circ}$ 
Ces deux morphismes sont compatibles à la suite exacte précédente

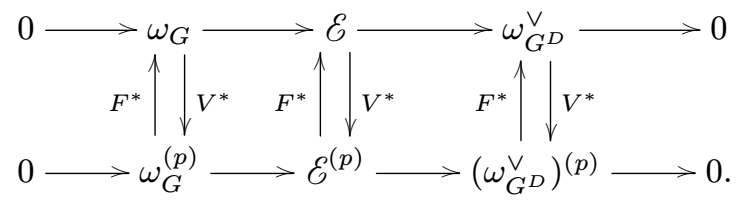

En restriction à $\omega_{G}^{(p)}$ le morphisme $F^{*}$ est nul. Il se factorise donc en un morphisme

$$
F^{*}:\left(\omega_{G^{D}}^{\vee}\right)^{(p)} \longrightarrow \mathcal{E} .
$$

Le morphisme $V^{*}:\left(\omega_{G^{D}}^{\vee}\right)^{(p)} \longrightarrow \omega_{G^{D}}^{\vee}$ est nul et donc

$$
V^{*}: \mathcal{E} \longrightarrow \omega_{G}^{(p)}
$$

Il y a une suite exacte de complexes parfaits de $\theta_{X}$-modules (dans le diagramme qui suit les complexes sont les lignes horizontales)

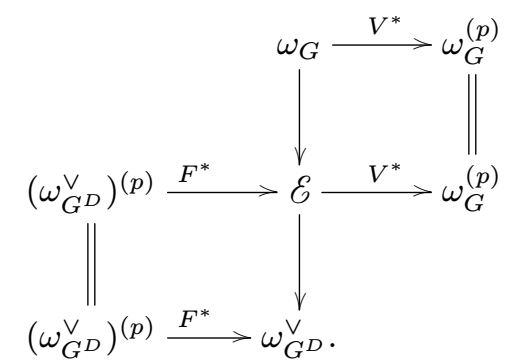

Notons $0 \rightarrow C_{1} \longrightarrow C_{2} \rightarrow C_{3} \rightarrow 0$ cette suite. Chacun de ces complexes parfaits est de rang nul. Le diagramme précédent induit donc un isomorphisme ([27])

$$
\operatorname{det}\left(C_{1}\right) \otimes \operatorname{det}\left(C_{3}\right) \stackrel{\sim}{\longrightarrow} \operatorname{det}\left(C_{2}\right) .
$$

On a $\operatorname{det}\left(C_{1}\right)=\omega_{G}^{\otimes(p-1)}$ et $\operatorname{det}\left(C_{3}\right)=\omega_{G_{D}}^{\otimes(1-p)}$. Remarquons maintenant qu'étant donné que $\mathscr{G}$ est un groupe de Barsotti-Tate tronqué d'échelon 1, le complexe $C_{2}$ est exact. Il y a donc un isomorphisme canonique $\operatorname{det}\left(C_{2}\right) \stackrel{\sim}{\longrightarrow} \theta_{X}$ qui induit l'isomorphisme cherché $\omega_{G}^{\otimes(p-1)} \simeq \omega_{G^{D}}^{\otimes(p-1)}$.

Supposons maintenant que sur un ouvert schématiquement dense de $X$ les complexes $C_{1}$ et $C_{3}$ soient acycliques. Les invariants $\mathrm{Ha}(G)$ et $\mathrm{Ha}\left(G^{D}\right)$ sont donc des diviseurs de Cartier. Avec les notations du chapitre II de [27] cela induit une égalité de diviseurs de Cartier

$$
0=\operatorname{Div}\left(C_{2}\right) \simeq \operatorname{Div}\left(C_{1}\right)+\operatorname{Div}\left(C_{3}\right)=\mathrm{Ha}\left(G^{D}\right)-\mathrm{Ha}(G) .
$$

On en déduit le résultat sous l'hypothèse précédente. Passons au cas général. Soit $Y$ le foncteur qui à un $\mathbb{F}_{p}$-schéma $S$ associe les classes d'isomorphismes de couples $(H, \alpha)$ où $H$ est un groupe de Barsotti-Tate tronqué d'échelon 1, de hauteur $h$ et de dimension $d$ et $\alpha: \theta_{S}^{p^{h}} \stackrel{\sim}{\longrightarrow} \vartheta_{H}$ un isomorphisme de $\vartheta_{S}$-modules. On vérifie aisément qu'il est représentable par un $\mathbb{F}_{p}$-schéma de type fini. D'après le point a) du théorème 4.4 de [24], ce schéma est lisse sur $\operatorname{Spec}\left(\mathbb{F}_{p}\right)$. La proposition A.2.2.1 de [24] implique que l'ouvert d'ordinarité dans $Y$ est dense. Il est donc schématiquement dense. Soit $H$ le groupe de Barsotti-Tate 
tronqué universel sur $Y$. D'après l'étude précédente on a $\mathrm{Ha}(H)=\mathrm{Ha}\left(H^{D}\right)$. Or il existe un diagramme de schémas

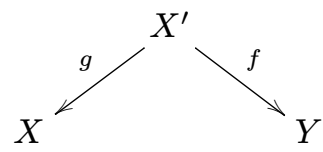

tel que $g$ soit fidèlement plat (un $\mathrm{GL}_{p^{h}}$-torseur) et

$$
g^{*} \mathscr{G} \simeq f^{*} H
$$

On en déduit que $g^{*} \mathrm{Ha}(\mathscr{G})=g^{*} \mathrm{Ha}\left(\mathscr{G}^{D}\right)$. Le morphisme $g$ étant fidèlement plat on en déduit que $\mathrm{Ha}(\mathscr{G})=\mathrm{Ha}\left(\mathscr{G}^{D}\right)$.

Remarque 1. - La preuve de la proposition précédente utilise le fait que $G$ est un groupe de Barsotti-Tate tronqué. Un tel type d'énoncé n'existe pas pour des groupes plats finis plus généraux. Plus précisément, soit $G$ un schéma en groupes de Oort-Tate sur $\operatorname{Spec}\left(\theta_{K}\right)$ ([35]). On a $G \simeq \operatorname{Spec}\left(\theta_{K}[T] /\left(T^{p}-\delta T\right)\right)$ et $G^{D} \simeq \operatorname{Spec}\left(\theta_{K}[T] /\left(T^{p}-\gamma T\right)\right)$ où $\gamma, \delta \in \theta_{K}$ sont tels que $\gamma \delta$ est une somme de Gauss de valuation $p$-adique 1 . Le Verschiebung $V:\left(G \otimes \theta_{K, 1}\right)^{(p)} \rightarrow G \otimes \vartheta_{K, 1}$ est induit par le morphisme d'algèbre

$$
\begin{aligned}
\vartheta_{K, 1}[T] /\left(T^{p}-\delta T\right) & \longrightarrow \vartheta_{K, 1}[T] /\left(T^{p}-\delta^{p} T\right) \\
T & \longmapsto \gamma T .
\end{aligned}
$$

Appliquant cela à $G^{D}$, on voit que le morphisme $F_{G *}: \omega_{G^{D}} \rightarrow \omega_{G^{D}}^{(p)}$ s'identifie à

$$
\begin{aligned}
\vartheta_{K} / \gamma \vartheta_{K} & \longrightarrow \vartheta_{K} /\left(p, \gamma^{p}\right) \\
\overline{1} & \longmapsto \delta \bmod \left(p, \gamma^{p}\right) .
\end{aligned}
$$

On en déduit que pour $F_{G *}: \omega_{G^{D}} \rightarrow \omega_{G^{D}}^{(p)}$ et $F_{G^{D *}}: \omega_{G} \rightarrow \omega_{G}^{(p)}$ on a

$$
\begin{aligned}
\operatorname{deg}\left(\operatorname{coker}\left(F_{G_{*}}\right)\right) & =\inf \{v(\delta), p v(\gamma)\} \\
\operatorname{deg}\left(\operatorname{coker}\left(F_{G^{D}}\right)\right) & =\inf \{v(\gamma), p v(\delta)\}
\end{aligned}
$$

qui ne sont pas égaux en général.

2.2.4. Calcul explicite sur les espaces de déformation. - Soit $H$ un groupe $p$-divisible de hauteur $h$ et de dimension $d$ sur $\overline{\mathbb{F}}_{p}$. Soit $(M, F, V)$ son cristal de Dieudonné covariant. On a donc Lie $H=M / V M$. Fixons une base $\left(e_{1}, \ldots, e_{h}\right)$ du $W\left(\overline{\mathbb{F}}_{p}\right)$-module libre $M$ telle que $e_{1}, \ldots, e_{d}$ induise une base de $M / V M$ et $e_{d+1}, \ldots, e_{h} \in V M$. Soit $A \in \mathrm{GL}_{h}\left(W\left(\overline{\mathbb{F}}_{p}\right)\right)$ la matrice telle que

$$
\left(\begin{array}{c}
F e_{1} \\
\vdots \\
F e_{d} \\
V^{-1} e_{d+1} \\
\vdots \\
V^{-1} e_{h}
\end{array}\right)=A\left(\begin{array}{c}
e_{1} \\
\vdots \\
\vdots \\
\vdots \\
e_{h}
\end{array}\right)
$$


Soit $\mathfrak{X}$ l'espace de déformation par isomorphismes de $H$, un $\operatorname{Spf}\left(W\left(\overline{\mathbb{F}}_{p}\right)\right)$-schéma formel. Soit

$$
B=\left(\begin{array}{cc}
I_{d} & {\left[x_{i j}\right]} \\
0 & I_{h-d}
\end{array}\right) A \in \mathrm{GL}_{h}\left(W\left(W\left(\overline{\mathbb{F}}_{p}\right) \llbracket x_{i j} \rrbracket_{\substack{1 \leq i \leq d \\
1 \leq j \leq h-d}}\right)\right) .
$$

D'après la formule (86) page 174 de [41] il existe un isomorphisme $\operatorname{Spf}\left(W\left(\overline{\mathbb{F}}_{p}\right) \llbracket x_{i j} \rrbracket\right) \stackrel{\sim}{\longrightarrow} \mathfrak{X}$ et une base $\left(\epsilon_{1}, \ldots, \epsilon_{h}\right)$ du Display de la déformation universelle telle que $B$ soit la matrice exprimant $\left(F \epsilon_{1}, \ldots, F \epsilon_{d}, V^{-1} \epsilon_{d+1}, \ldots, V^{-1} \epsilon_{h}\right)$ en fonction de $\left(\epsilon_{1}, \ldots, \epsilon_{h}\right)$. Soit $\tilde{B} \in \mathrm{GL}_{h}\left(W\left(\overline{\mathbb{F}}_{p}\right) \llbracket x_{i j} \rrbracket\right)$ la réduction de $B$ via $\left.W\left(W\left(\overline{\mathbb{F}}_{p}\right) \llbracket x_{i j} \rrbracket\right) \rightarrow W\left(\overline{\mathbb{F}}_{p}\right) \llbracket x_{i j} \rrbracket\right)$. Si

$$
A=\left(\begin{array}{ll}
A_{1} & A_{2} \\
A_{3} & A_{4}
\end{array}\right)
$$

avec $A_{1}$ de taille $(d, d)$ alors

$$
\tilde{B}=\left(\begin{array}{cr}
A_{1}+\left(x_{i j}\right)_{i, j} \cdot A_{3} * \\
* & *
\end{array}\right) .
$$

Soit alors $f=\operatorname{det}\left(A_{1}+\left(x_{i j}\right)_{i, j} . A_{3}\right) \in W\left(\overline{\mathbb{F}}_{p}\right) \llbracket x_{i j} \rrbracket$. Cette fonction $f$ définit une fonction rigide analytique sur la fibre générique de $\mathfrak{X}$. Alors,

$$
\begin{aligned}
\mathrm{Ha}: \mathfrak{X}^{\mathrm{an}} & \longrightarrow[0,1] \\
x & \longmapsto \inf \{v(f(x)), 1\} .
\end{aligned}
$$

Le cas de l'espace de Lubin-Tate est particulièrement simple. Supposons donc $d=1$ et $H$ formel. On peut choisir

$$
A=\left(\begin{array}{cccc}
0 & \cdots & 0 & 1 \\
1 & & \\
& \ddots & \\
& & 1
\end{array}\right)
$$

et donc $f=x_{1}$ où $\operatorname{Spf}\left(W\left(\overline{\mathbb{F}}_{p}\right) \llbracket x_{1}, \ldots, x_{h-1} \rrbracket\right) \stackrel{\sim}{\longrightarrow} \mathfrak{X}$ (avec les notations précédentes, $x_{j}:=x_{1 j}$ ). On vérifie que le module de Cartier associé au Display de la déformation universelle ([41] prop. 90) possède pour $V$-base un élément $e$ qui satisfait à l'équation fonctionnelle

$$
F . e=\sum_{j=0}^{h-1} V^{j}\left[x_{j+1}\right] e+V^{h} e .
$$

Si $H^{\text {univ }}$ désigne la déformation universelle sur $\mathfrak{X}=\operatorname{Spf}(R), e \in H^{\text {univ }}(R \llbracket T \rrbracket)$ est une courbe $p$-typique qui induit un isomorphisme $\operatorname{Spf}(R \llbracket T \rrbracket) \stackrel{\sim}{\longrightarrow} H^{\text {univ }}$, c'est-à-dire une loi de groupe formel associée à la déformation universelle. L'équation fonctionnelle précédente se traduit en ce que le logarithme de la loi de groupe formel universelle précédente satisfait à l'équation fonctionnelle (5.4) de la section 5 de [23]. Cette loi de groupe formelle universelle est donc celle étudiée dans [23]. Pour celle-ci, le polygone de Newton de la multiplication par $p$ est donné par l'enveloppe convexe des points $(1,1),\left(p, v\left(x_{1}\right)\right), \ldots,\left(p^{h-1}, v\left(x_{h-1}\right)\right),\left(p^{h}, 0\right)$. 
De cette analyse on déduit que si $\mathscr{G}$ est un groupe formel $p$-divisible de dimension 1 et hauteur $h$ sur $\theta_{K}$ et $\operatorname{Newt}(\mathscr{G}[p]):\left[1, p^{h}\right] \rightarrow \mathbb{R}_{+}$désigne le polygone de Newton de la multiplication par $p \operatorname{sur} \mathscr{G}$ alors on a toujours

$$
\mathrm{Ha}(\mathscr{G}[p]) \geq \operatorname{Newt}(\mathscr{G}[p])(p)
$$

avec égalité dès que $\operatorname{Newt}(\mathscr{G}[p])$ possède un point de rupture en l'abscisse $p$.

\subsection{Groupes de Barsotti-Tate tronqués ordinaires}

Nous avons défini précédemment l'invariant de Hasse d'un groupe de Barsotti-Tate tronqué d'échelon 1 dans divers contextes. Pour un groupe de Barsotti-Tate d'échelon quelconque, ou bien un groupe de Barsotti-Tate, on définit son invariant de Hasse comme étant celui de ses points de $p$-torsion.

Définition 1. - Un groupe de Barsotti-Tate tronqué sur un schéma formel p-adique est dit ordinaire s'il s'écrit comme une extension d'un groupe étale par un groupe de type multiplicatif.

Lorsque $G$ est ordinaire, il s'écrit canoniquement comme une extension d'un groupe étale par un groupe de type multiplicatif, il s'agit de la suite

$$
0 \longrightarrow G^{0} \longrightarrow G \longrightarrow G^{\text {ét }} \longrightarrow 0 .
$$

Soient $K \mid \mathbb{Q}_{p}$ et $\mathfrak{X}$ comme précédemment. Soit $G$ un groupe de Barsotti-Tate tronqué sur $\mathfrak{X}$. Il est alors bien connu que les assertions suivantes sont équivalentes :

- Le groupe $G$ est ordinaire.

- Le groupe $G[p]$ est ordinaire.

- Pour tout $x \in \mathfrak{X}^{\text {rig }}$, resp. $x \in \mathfrak{X}^{\text {an }}, G_{x}$ est ordinaire.

- Pour tout $x \in \mathfrak{X}^{\text {rig }}$, resp. $x \in \mathfrak{X}^{\text {an }}, \mathrm{Ha}\left(G_{x}\right)=0$.

- Le groupe $G \times \mathfrak{X} \mathfrak{X}_{\text {red }}$ est ordinaire.

- Pour tout point fermé $x \in \mathfrak{X}_{r e d}, G_{k(x)}$ est ordinaire.

- L'application $\psi_{G[p]}: \omega_{G[p]^{D}} \longrightarrow \omega_{G[p]^{D}}^{(p)}$ est un isomorphisme, i.e. $\widetilde{\mathrm{Ha}}(G)$ induit une trivialisation $\theta_{\mathfrak{X}} / p \theta_{\mathfrak{X}} \stackrel{\sim}{\longrightarrow} \operatorname{det}\left(\omega_{G[p]^{D}}\right)^{\otimes(p-1)}$.

Définition 2. - On note $\mathfrak{X}_{\text {ord }}^{\text {an }}$, resp. $\mathfrak{X}_{\text {ord }}^{\text {rig }}$, le lieu ordinaire. Pour $\left.\left.\epsilon \in\right] 0,1\right] \cap v\left(\bar{K}^{\times}\right)$, on note $\mathfrak{X}_{\text {ord }}^{\text {an }}(\epsilon)$, resp. $\mathfrak{X}_{\text {ord }}^{\text {rig }}(\epsilon)$, le lieu où l'invariant de Hasse est plus petit que $\epsilon$. On note $\mathfrak{X}_{\text {ord }}^{\text {an }}(\stackrel{\circ}{)})$ et $\mathfrak{X}_{\mathrm{ord}}^{\mathrm{rig}}(\stackrel{\circ}{\epsilon})$ les lieux où l'invariant de Hasse est strictement plus petit que $\epsilon$.

D'après le lemme $1, \mathfrak{X}_{\text {ord }}^{\text {an }}$ est un domaine analytique fermé dans $\mathfrak{X}^{\text {an }}$ associé à l'ouvert admissible quasicompact $\mathfrak{X}_{\text {ord }}^{\text {rig }}$. Le lieu d'ordinarité de la réduction modulo $p$ de $G$ définit un ouvert $\mathfrak{U}=\mathfrak{X} \backslash V\left(\operatorname{HaI}_{\mathfrak{I}}(G)\right)$ de $\mathfrak{X}$. On a alors $\mathfrak{X}_{\text {ord }}^{\text {an }}=\mathfrak{U}^{\text {an }}$ et $\mathfrak{X}_{\text {ord }}^{\text {rig }}=\mathfrak{U}^{\text {rig }}$.

Le lieu $\mathfrak{X}_{\text {ord }}^{\text {an }}(\epsilon)$, resp. $\mathfrak{X}_{\text {ord }}^{\text {rig }}(\epsilon)$, est un domaine analytique fermé dans $\mathfrak{X}^{\text {an }}$, resp. un ouvert admissible quasicompact dans $\mathfrak{X}^{\text {rig }}$. Néanmoins en général il faut procéder à un éclatement formel admissible de $\mathfrak{X}$ afin de faire apparaître ces lieux comme des ouverts de $\mathfrak{X}$ (éclatement qui dépend de $\epsilon$ ).

Le lieu $\mathfrak{X}_{\text {ord }}^{\text {an }}(\stackrel{\bullet}{\epsilon})$ est un ouvert de $\mathfrak{X}^{\text {an }}$ associé à l'ouvert admissible $\mathfrak{X}_{\text {ord }}^{\text {rig }}(\stackrel{\bullet}{\epsilon})$ (ouvert admissible qui n'est pas quasicompact en général).

$4^{\mathrm{e}}$ SÉRIE - TOME $44-2011-\mathrm{N}^{\mathrm{o}} 6$ 


\subsection{Caractérisation de l'ordinarité via les polygones de Harder-Narasimhan}

Rappelons que dans la théorie développée dans [16] les pentes des polygones de HarderNarasimhan sont à valeurs dans $[0,1]$. Si $G$ est un groupe de Barsotti-Tate tronqué d'échelon $n$, de hauteur $h$ et de dimension $d \operatorname{sur} \theta_{K}$ on a les formules

$$
\operatorname{deg}(G)=n d, \operatorname{ht}(G)=n h \text { et } \mu(G)=\frac{d}{h} .
$$

Le polygone de Harder-Narasimhan d'un tel groupe est alors en dessous du polygone concave de pentes 1 avec multiplicité $n d$ et 0 avec multiplicité $n(h-d)$. Rappelons de plus que pour ces filtrations de Harder-Narasimhan la partie de pente 1 correspond au plus grand sous-groupe de type multiplicatif et celle de pente 0 au plus grand quotient étale. Le lemme qui suit est donc immédiat.

Lemme 2. - Soit $G$ un groupe de Barsotti-Tate tronqué d'échelon $n$, de dimension d et de hauteur $h$ sur $\theta_{K}$. Il est ordinaire si et seulement si le polygone $\operatorname{HN}(G)$ est le polygone concave de pentes 1 et 0 avec multiplicités nd et $n(h-d)$, si et seulement si $\operatorname{HN}(G)(d)=d$. Si c'est le cas, la suite

$$
0 \longrightarrow G^{0} \longrightarrow G \longrightarrow G^{e ́ t} \longrightarrow 0
$$

est la filtration de Harder-Narasimhan de G.

\subsection{Caractérisation de l'ordinarité via l'application de Hodge-Tate de $G$}

Soit $G$ fini et plat $\operatorname{sur} \theta_{K}$. On note encore

$$
\alpha_{G}: G\left(\theta_{\bar{K}}\right) \longrightarrow \omega_{G^{D}} \otimes \theta_{\bar{K}}
$$

le morphisme de groupes induit par $\alpha_{G}: G \longrightarrow \underline{\omega}_{G^{D}}$. C'est ce qu'on appelle l'application de Hodge-Tate de $G$. On considérera également le morphisme de $\theta_{\bar{K}}$-modules

$$
\alpha_{G} \otimes 1: G\left(\theta_{\bar{K}}\right) \otimes_{\mathbb{Z}} \theta_{\bar{K}} \longrightarrow \omega_{G^{D}} \otimes \theta_{\bar{K}} .
$$

Le lemme qui suit est immédiat.

Lemme 3. - Soit $G$ un schéma en groupes fini et plat sur $\theta_{K}$ annulé par $p$. L'image de l'application de Hodge-Tate $\alpha_{G}$ de $G$ est contenue dans le $\mathbb{F}_{p}$-espace vectoriel

$$
\left\{x \in \omega_{G^{D}} \otimes \theta_{\bar{K}} \mid \psi_{G}(x)=x \otimes 1\right\}
$$

où pour $x \in \omega_{G^{D}} \otimes \vartheta_{\bar{K}}$ on note $x \otimes 1 \in\left(\omega_{G^{D}} \otimes \vartheta_{\bar{K}}\right) \otimes_{\vartheta_{K, 1}, \text { Frob }} \theta_{K, 1}=\left(\omega_{G^{D}} \otimes \vartheta_{\bar{K}}\right)^{(p)}$.

On remarquera que, lorsque $G$ est annulé par $p$, l'application de Hodge-Tate précédente se factorise via l'application de réduction modulo $p$ :

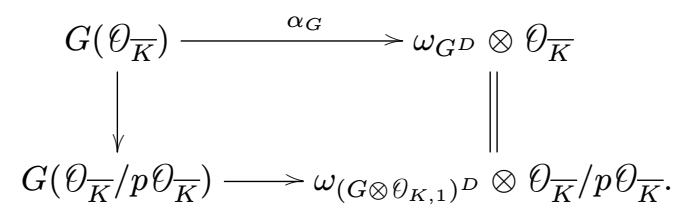


Lemme 4. - Un groupe de Barsotti-Tate tronqué G d'échelon 1 sur $\theta_{K}$ est ordinaire si et seulement si $\alpha_{G} \otimes 1$ est surjective. Si c'est le cas, l'application $\alpha_{G}$ détermine complètement la suite $0 \rightarrow G^{0} \longrightarrow G \rightarrow G^{e ́ t} \rightarrow 0$ puisqu'alors

$$
G^{0}\left(\theta_{\bar{K}}\right)=\operatorname{ker} \alpha_{G}
$$

Démonstration. - Si $G$ est ordinaire, on vérifie aussitôt par un calcul sur les groupes multiplicatifs et étales que $\alpha_{G} \otimes 1$ est surjective et $G^{0}\left(\theta_{\bar{K}}\right)=\operatorname{ker}\left(\alpha_{G}\right)$.

Réciproquement supposons $\alpha_{G} \otimes 1$ surjective. D'après le lemme 3, cela implique que le $\vartheta_{\bar{K}}$-module $\omega_{G^{D}}^{(p)} \otimes \theta_{\bar{K}}$ est engendré par des éléments dans l'image de $\psi_{G}$ et que donc $\psi_{G}$ est surjective après extension des scalaires à $\theta_{\bar{K}}$ et $\psi_{G}$ l'est donc.

\section{La filtration canonique : une borne non effective}

Les résultats de cette section ne seront pas utilisés dans la suite. On montre comment obtenir simplement des bornes non effectives sur l'invariant de Hasse impliquant l'existence d'un sous-groupe canonique en niveau quelconque au sens des filtrations de Harder-Narasimhan. Dans le cas des schémas abéliens et pour d'autres filtrations ce type de résultat a été obtenu par Conrad dans [10].

\subsection{Sur le champ des $B T_{n}$}

Soit $n \geq 1$ un nombre entier. On munit la catégorie des $\operatorname{Spec}\left(\mathbb{Z}_{p}\right)$-schémas de la topologie lisse. Soit

$$
\mathrm{BT}_{n}
$$

le champ des groupes de Barsotti-Tate tronqués d'échelon $n$. Il s'écrit comme une union disjointe de sous-champs ouverts

$$
\mathrm{BT}_{n}=\coprod_{h \in \mathbb{N}} \mathrm{BT}_{n, h}
$$

où $\mathrm{BT}_{n, h}$ est le sous-champ où la hauteur du groupe de Barsotti-Tate tronqué est $h$. Il y a une identification

$$
\mathrm{BT}_{n, h} \otimes \mathbb{Q}_{p}=B G L_{h}\left(\mathbb{Z} / p^{n} \mathbb{Z}\right) .
$$

Soit $X_{n, h}$ le foncteur qui à un $\mathbb{Z}_{p}$-schéma $S$ associe les classes d'isomorphismes de couples $(G, \alpha)$, où $G$ est un groupe de Barsotti-Tate tronqué d'échelon $n$ et de hauteur $h$ et

$$
\alpha: \theta_{S}^{p^{n h}} \stackrel{\sim}{\longrightarrow} \theta_{G}
$$

est un isomorphisme de $\theta_{S}$-modules. Il y a une action du groupe algébrique $\mathrm{GL}_{p^{n h}} \operatorname{sur} X_{n, h}$ par action sur la rigidification $\alpha$ ainsi qu'un morphisme $\mathrm{GL}_{p^{n h}}$-invariant

$$
X_{n, h} \longrightarrow \mathrm{BT}_{n, h} \text {. }
$$

D'après la proposition 1.8 de [40], $X_{n, h}$ est représentable par un $\mathbb{Z}_{p}$-schéma de type fini. Le morphisme précédent induit de plus un isomorphisme

$$
\left[\mathrm{GL}_{p^{n h}} \backslash X_{n, h}\right] \stackrel{\sim}{\longrightarrow} \mathrm{BT}_{n, h} .
$$

En particulier, $\mathrm{BT}_{n, h}$ est un champ algébrique de type fini sur $\operatorname{Spec}\left(\mathbb{Z}_{p}\right)$.

4 e SÉRIE - TOME $44-2011-$ No $^{\circ}$ 
Considérons la catégorie des $\operatorname{Spf}\left(\mathbb{Z}_{p}\right)$ schémas formels $p$-adiques, c'est-à-dire les schémas formels $\mathfrak{X}$ tels que $p \vartheta_{\mathfrak{X}}$ soit un idéal de définition de $\mathfrak{X}$. Par définition, un morphisme lisse de schémas formels $p$-adiques est un morphisme dont la réduction modulo $p^{k}$ est un morphisme lisse de schémas pour tout $k \geq 1$. On munit la catégorie des schémas formels $p$-adiques de la topologie lisse. Soit

$$
\widehat{\mathrm{BT}}_{n, h}
$$

le champ sur ce site classifiant les groupes de Barsotti-Tate tronqués d'échelon $n$ et de hauteur $h$. Il y a une décomposition en union disjointe de sous-champs ouverts

$$
\widehat{\mathrm{BT}}_{n, h}=\coprod_{0 \leq d \leq h} \widehat{\mathrm{BT}}_{n, h, d}
$$

où $d$ désigne la dimension du groupe de Barsotti-Tate tronqué. Soit $\widehat{X}_{n, h}$ le complété $p$-adique de $X_{n, h}$. Il y a une décomposition $\mathrm{GL}_{p^{n h} \text {-invariante }}$

$$
\widehat{X}_{n, h}=\coprod_{0 \leq d \leq h} \widehat{X}_{n, h, d}
$$

et un isomorphisme

$$
\left[\mathrm{GL}_{p^{n h}} \backslash \widehat{X}_{n, h, d}\right] \stackrel{\sim}{\longrightarrow} \widehat{\mathrm{BT}}_{n, h, d} .
$$

Remarquons que, d'après le théorème 4.4 de [24], le champ $\widehat{\mathrm{BT}}_{n, h, d}$ est formellement lisse sur $\operatorname{Spf}\left(\mathbb{Z}_{p}\right)$ et donc $\widehat{X}_{n, h, d}$ est un $\operatorname{Spf}\left(\mathbb{Z}_{p}\right)$-schéma formel lisse.

\subsection{Une borne non effective}

Proposition 3. - Soient $0 \leq d<$ h et $n \geq 1$ des nombres entiers. Il existe $\epsilon(n, d, h)>0$ tel que si $K$ est une extension valuée complète de $\mathbb{Q}_{p}$ pour une valuation de rang 1 , G est un groupe de Barsotti-Tate tronqué sur $\emptyset_{K}$ d'échelon $n$, de hauteur $h$ et de dimension d vérifiant

$$
\mathrm{Ha}(G)<\epsilon(n, d, h)
$$

alors la filtration de Harder-Narasimhan de $G$ possède un cran $C$ tel que $C\left(\theta_{\bar{K}}\right)$ soit un $\mathbb{Z} / p^{n} \mathbb{Z}$-module libre de rang $d$.

Démonstration. - Commençons par le cas $n=1$. Soit $E$ le groupe universel sur $\widehat{X}_{1, h, d}$. D'après le théorème 3 de [16], la fonction

$$
\begin{aligned}
\left|\widehat{X}_{1, h, d}^{\text {an }}\right| & \longrightarrow\{\text { Polygones }:[0, h] \rightarrow[0, d]\} \\
x & \longmapsto \operatorname{HN}\left(E[p]_{x}\right)
\end{aligned}
$$

est continue, l'ensemble des polygones étant munis de la topologie de la convergence uniforme. De plus, d'après le lemme 2 ,

$$
\left|\left(\widehat{X}_{1, h, d}^{\text {an }}\right)_{\text {ord }}\right|=\mathrm{HN}^{-1}\left(\left\{\mathscr{P}_{\text {ord }}\right\}\right)
$$

où $\mathscr{P}_{\text {ord }}$ est le polygone concave de pentes 1 et 0 avec multiplicités $d$ et $h-d$. Il existe un voisinage $U$ de $\mathscr{P}_{\text {ord }}$ dans lequel tout polygone a un point de rupture en l'abscisse $d$. L'ensemble

$$
\begin{gathered}
\operatorname{HN}^{-1}(U) \\
\text { est alors un voisinage de }\left|\left(\widehat{X}_{1, h, d}^{\text {an }}\right)_{\text {ord }}\right| \text {. Puisque }\left|\widehat{X}_{1, h, d}^{\text {an }}\right| \text { est compact, l'application } \\
\qquad \mathrm{Ha}:\left|\widehat{X}_{1, h, d}^{\text {an }}\right| \longrightarrow[0,1]
\end{gathered}
$$


est propre. De cela on déduit que

$$
\left\{\mathrm{Ha}^{-1}([0, \epsilon[) \mid 0<\epsilon<1\}\right.
$$

forment une base de voisinage de $\mathrm{Ha}^{-1}(\{0\})=\left|\left(\widehat{X}_{1, h, d}^{\text {an }}\right)_{\text {ord }}\right|$ et qu'il existe donc $\epsilon(d, h)>0$ tel que

$$
\left(\widehat{X}_{1, h, d}^{\text {an }}\right)_{\text {ord }}(\stackrel{\circ}{\epsilon}(d, h)) \subset U \text {. }
$$

Lorsque $n=1$ on peut alors prendre $\epsilon(1, d, h):=\epsilon(d, h)$.

Traitons maintenant le cas $n \geq 2$. Soit $E$ le groupe universel sur $\widehat{X}_{n, h, d}$. Par le même raisonnement que précédemment sur l'espace $\widehat{X}_{n, h, d}^{\text {an }}$, on vérifie qu'il existe $\epsilon>0$ tel pour tout $x \in\left(\widehat{X}_{n, h, d}^{\text {an }}\right)_{\text {ord }}(\epsilon)$, le groupe spécialisé $E_{x}$ possède un cran de sa filtration de HarderNarasimhan qui est de hauteur $n d$. L'espace analytique $\left(\widehat{X}_{n, h, d}^{\text {an }}\right)_{\text {ord }}(\epsilon)$ est compact. Il possède donc un nombre fini de composantes connexes qui sont ouvertes et fermées. En effet, si $\mathscr{G}$ est une algèbre affinoïde, les composantes connexes de l'espace de Berkovich $\mathcal{M}(\mathscr{C})$ sont en bijection avec les composantes connexes de $\operatorname{Spec}(\mathscr{Q})$ et il s'en suit que tout espace de Berkovich compact possède un nombre fini de composantes connexes. Soit

$$
\left(\widehat{X}_{n, h, d}^{\mathrm{an}}\right)_{\mathrm{ord}}(\epsilon)=\coprod_{i \in I} Y_{i}
$$

la décomposition associée avec $I$ fini et pour tout $i \in I, Y_{i}$ connexe. Soit $J \subset I$ le sousensemble défini par

$$
J=\left\{i \in I \mid Y_{i} \cap\left(\widehat{X}_{n, h, d}^{\text {an }}\right)_{\text {ord }} \neq \varnothing\right\} .
$$

Pour tout $i \in J$, choisissons $\bar{x}_{i}$ un point géométrique à valeurs dans $Y_{i} \cap\left(\widehat{X}_{n, h, d}^{\text {an }}\right)_{\text {ord }}$. Soit pour $i \in J$

$$
\rho_{i}: \pi_{1}\left(Y_{i}, \bar{x}_{i}\right) \longrightarrow \operatorname{Aut}\left(E_{\bar{x}_{i}}\right)
$$

la représentation de monodromie associée au groupe étale fini $E^{\text {an }}$. Soit $C_{i} \subset E_{\bar{x}_{i}}$ le sousgroupe de $E_{\bar{x}_{i}}$ associé au cran de hauteur $n d$ de la filtration de Harder-Narasimhan de la spécialisation de $E$ sur l'anneau des entiers du point associé au point géométrique $\bar{x}_{i}$. D'après le théorème 4 de [16], ce sous-groupe est invariant sous la représentation de monodromie $\rho_{i}$. Puisque $\bar{x}_{i}$ est à valeurs dans le lieu ordinaire, $C_{i}$ est un $\mathbb{Z} / p^{n} \mathbb{Z}$-module libre de rang $d$. On en déduit qu'en tout point $x$ de $Y_{i}$, le cran de la filtration de Harder-Narasimhan de $E_{x}$ de rang $n d$ a pour fibre générique géométrique un groupe libre de rang $n d$.

Constatons maintenant que $\bigcup_{i \in J} Y_{i}$ forme un voisinage de $\left(\widehat{X}_{n, h, d}^{\text {an }}\right)_{\text {ord }}$. Il existe donc $\epsilon(n, d, h)>0$ tel que

$$
\left(\widehat{X}_{n, h, d}^{\mathrm{an}}\right)_{\text {ord }}(\epsilon(n, d, h)) \subset \bigcup_{i \in J} Y_{i}
$$

On conclut aisément.

\section{Sur les filtrations des schémas en groupes finis et plats}

Dans cette section nous rappelons et établissons quelques propriétés de certaines filtrations des schémas en groupes finis et plats définies par Abbes-Saito et Andreatta-Gasbarri. Les points nouveaux sont la proposition 6 qui est une démonstration élémentaire du théorème 1.6 de [36] ainsi que la proposition 5 qui relie la filtration de congruence à la fonction 
degré définie dans [16]. Ces résultats seront utilisés dans la section 6. On note $\Gamma=v\left(\bar{K}^{\times}\right)$. Soit $G$ un schéma en groupes fini et plat sur $\theta_{K}$ d'ordre une puissance de $p$.

\subsection{Les différentes filtrations en jeu}

\subsubsection{La filtration de ramification inférieure naïve}

Définition 3. - Pour $\lambda \in \Gamma, \lambda>0$, on pose

$$
G_{\mathrm{rn}}^{\lambda}\left(\theta_{\bar{K}}\right)=\operatorname{ker}\left(G\left(\theta_{\bar{K}}\right) \longrightarrow G\left(\theta_{\bar{K}, \lambda}\right)\right) .
$$

Cela définit une filtration Gal $(\bar{K} \mid K)$-invariante de $G\left(\Theta_{\bar{K}}\right)$ et donc une filtration du groupe étale $G \otimes K$. On note $\left(G_{\mathrm{rn}}^{\lambda}\right)_{\lambda>0}$ la filtration obtenue par adhérence schématique dans $G$, que l'on appelle filtration de ramification naïve (il est sous-entendu dans cette notation que $\lambda$ parcourt des éléments de $\Gamma$ ).

La filtration $\left(G_{\mathrm{rn}}^{\lambda}\right)_{\lambda>0}$ est décroissante. Le sous-groupe $G_{\mathrm{rn}}^{\lambda}$ coïncide avec la composante connexe neutre de $G$ lorsque $\lambda$ est suffisamment proche de 0 . On vérifie de plus aisément que les sauts de cette filtration sont dans $\Gamma$. Cette filtration est compatible à la restriction à un sous-groupe : si $G^{\prime} \subset G$ est un sous-groupe plat fini de $G$, alors, pour $\lambda \in \Gamma, \lambda>0$,

$$
G_{\mathrm{rn}}^{\prime \lambda} \otimes K=\left(G^{\prime} \otimes K\right) \cap\left(G_{\mathrm{rn}}^{\lambda} \otimes K\right) .
$$

\subsubsection{La filtration de ramification d'Abbes-Saito}

DéfINITION 4. - On note $\left(G_{\mathrm{AS}}^{\lambda}\right)_{\lambda \in \Gamma, \lambda>0}$ la filtration de ramification définie par Abbes et Saito ([2], [1]).

La définition d'Abbes et Saito concerne le cas où la valuation $v$ de $K$ est discrète. Néanmoins, la définition s'étend aussitôt au cas de valuation quelconque. D'après le théorème 5.1 de [2], lorsque la valuation est discrète, les sauts de cette filtration sont des nombres rationnels. L'auteur ignore si cette assertion s'étend au cas de valuation quelconque, i.e. si les sauts de cette filtration sont dans $\Gamma$ (nous n'utiliserons de toutes façons pas ce type d'assertion).

D'après la proposition 2.8 de [36], cette filtration est compatible aux quotients. Plus précisément, si

$$
f: G^{\prime} \longrightarrow G
$$

est un épimorphisme de schémas en groupes finis et plats alors, pour tout $\lambda>0, \lambda \in \Gamma$,

$$
f_{\mid G_{\mathrm{AS}}^{\prime \lambda}}: G_{\mathrm{AS}}^{\prime \lambda}\left(\theta_{\bar{K}}\right) \longrightarrow G_{\mathrm{AS}}^{\lambda}\left(\theta_{\bar{K}}\right)
$$

est surjectif. 
4.1.3. La filtration par les sous-groupes de congruence d'Andreatta-Gasbarri ([3]). - Pour $u \in \vartheta_{\bar{K}}$ vérifiant $v(u) \leq \frac{1}{p-1}$, on peut définir ([3] §5) un schéma en groupes fini et plat de hauteur $1, C_{u} \operatorname{sur} \operatorname{Spec}\left(\Theta_{\bar{K}}\right)$ d'algèbre $\theta_{\bar{K}}[T] /\left(P_{u}(T)\right)$ où

$$
P_{u}(T):=\frac{(1+u T)^{p}-1}{u^{p}} .
$$

Via cette identification, la section neutre de $C_{u}$ correspond à $T=0$. Il y a donc une identification

$$
\omega_{C_{u}} \simeq \theta_{\bar{K}} / p u^{1-p} \vartheta_{\bar{K}} .
$$

De cela on déduit qu'avec les notations de [33] (auxquelles l'auteur est plus habitué)

$$
C_{u} \simeq \operatorname{Spec}\left(\theta_{\bar{K}}[T] /\left(T^{p}-\delta T\right)\right)
$$

où $v(\delta)=1-(p-1) v(u)$. La famille $\left(C_{u}\right)_{u \in \vartheta_{\bar{K}}, v(u) \leq \frac{1}{p-1}}$ n'est qu'une reparamétrisation de la classification de Oort-Tate ([35]). Elle est de plus munie de morphismes canoniques non nuls, si $v\left(u_{1}\right) \geq v\left(u_{2}\right)$,

$$
\eta_{u_{1}, u_{2}} \in \operatorname{Hom}\left(C_{u_{1}}, C_{u_{2}}\right)
$$

vérifiant que si $v\left(u_{1}\right) \geq v\left(u_{2}\right) \geq v\left(u_{3}\right)$, alors

$$
\eta_{u_{1}, u_{3}}=\eta_{u_{2}, u_{3}} \circ \eta_{u_{1}, u_{2}} .
$$

Ces morphismes sont des isomorphismes en fibre générique. On a de plus

$$
\operatorname{Hom}\left(C_{u_{1}}, C_{u_{2}}\right)=\left\{\begin{array}{c}
(0) \text { si } v\left(u_{1}\right)<v\left(u_{2}\right) \\
\mathbb{F}_{p} . \eta_{u_{1}, u_{2}} \text { si } v\left(u_{1}\right) \geq v\left(u_{2}\right) .
\end{array}\right.
$$

Il y a un isomorphisme $C_{u_{1}} \simeq C_{u_{2}}$ si et seulement si $v\left(u_{1}\right)=v\left(u_{2}\right)$. On a l'égalité $C_{1}=\boldsymbol{\mu}_{p}$. Il y a donc un morphisme canonique

$$
\eta_{u, 1}: C_{u} \longrightarrow \boldsymbol{\mu}_{p}
$$

qui est un isomorphisme en fibre générique.

Définition 5. - Supposons $G$ annulé par p. Pour $\lambda \in \Gamma$ vérifiant $0 \leq \lambda \leq \frac{1}{p-1}$, on note

$$
G_{\text {cong }}^{\lambda}\left(\varphi_{\bar{K}}\right)=\operatorname{Hom}\left(G_{\vartheta_{\bar{K}}}^{D}, C_{u}\right) \stackrel{\eta_{u, 1 *}}{\longrightarrow} \operatorname{Hom}\left(G_{\vartheta_{\bar{K}}}^{D}, \mu_{p}\right)=G\left(\Theta_{\bar{K}}\right)
$$

où $u \in \theta_{\bar{K}}$ est n'importe quel élément de valuation $\lambda$. Cela forme un sous-groupe $\operatorname{Gal}(\bar{K} \mid K)$-invariant de $G\left(\vartheta_{\bar{K}}\right)$. On note $G_{\text {cong }}^{\lambda}$ le sous-groupe associé de $G$ par adhérence schématique du sous-groupe de $G \otimes K$ de $\bar{K}$-points $G_{\text {cong }}^{\lambda}\left(\theta_{\bar{K}}\right)$.

Cela définit une filtration décroissante $\left(G_{\text {cong }}^{\lambda}\right)_{\lambda \in \Gamma, 0 \leq \lambda \leq \frac{1}{p-1}}$ de $G$. Elle est compatible à la restriction à un sous-groupe.

EXEMPLE 1. - Le sous-groupe $G_{\mathrm{cong}}^{1 /(p-1)}$ est le plus grand sous-groupe de type multiplicatif de G. Le sous-groupe $\cup_{\lambda>0} G_{\text {cong }}^{\lambda}$ est la composante connexe neutre de $G$.

$4^{\mathrm{e}}$ SÉRIE - TOME $44-2011-\mathrm{N}^{\circ} 6$ 


\subsection{Comparaison des différentes filtrations pour les groupes annulés par $p$}

Le point de cette section est le suivant. Si l'on a défini une filtration sur les schémas en groupes finis et plats compatible à la restriction à un sous-groupe, alors celle-ci est complètement déterminée par les filtrations associées des groupes $E$ tels que $E\left(\theta_{\bar{K}}\right)$ soit cyclique, i.e. engendré par un seul élément. Il en est de même pour les filtrations compatibles aux quotients.

Maintenant, si on se restreint aux schémas en groupes annulés par $p$, de tels sousgroupes $E$ sont les groupes de hauteur 1. Or, d'après Oort et Tate ([35]), on peut effectuer des calculs explicites sur ceux-ci.

4.2.1. Filtration de ramification naïve et filtration par les sous-groupes de congruence. - Un calcul explicite sur les schémas en groupes de Oort-Tate fournit le lemme qui suit.

Lemme 5. - Soit E un schéma en groupes fini et plat de hauteur 1 sur $\emptyset_{\bar{K}}$ et $\lambda \in \Gamma, \lambda>0$. Alors

$$
E_{\mathrm{rn}}^{\lambda}=\left\{\begin{array}{c}
0 \text { si } \lambda>\frac{\operatorname{deg} E}{p-1} \\
E \text { si } \lambda \leq \frac{\operatorname{deg} E}{p-1} .
\end{array}\right.
$$

Proposition 4. - Supposons $G$ annulé par p. Alors, si $\lambda \in \Gamma, \lambda>\frac{1}{p-1}$, on $a G_{\mathrm{rn}}^{\lambda}=0$. De plus, si $0<\lambda \leq \frac{1}{p-1}$, on a l'égalité

$$
G_{\mathrm{rn}}^{\lambda}=G_{\mathrm{cong}}^{\lambda} .
$$

Démonstration. - Puisque nos deux filtrations sont compatibles à la restriction à un sousgroupe, il suffit, quitte à prendre l'adhérence schématique d'un sous- $\mathbb{F}_{p}$-espace vectoriel de dimension 1 de $G\left(\theta_{\bar{K}}\right)$, de démontrer la proposition pour un schéma en groupes de hauteur 1 $\operatorname{sur} \Theta_{\bar{K}}$. Or, si $E$ est un tel schéma en groupes, puisque $\operatorname{deg}(E) \leq 1$, d'après le lemme 5 on a $E_{\mathrm{rn}}^{\lambda}=0$ pour $\lambda>\frac{1}{p-1}$. De plus $E^{D} \simeq C_{u}$ où $u \in \Theta_{\bar{K}}$ vérifie $v(u)=\frac{\operatorname{deg} E}{p-1}$. On en déduit le résultat.

\subsubsection{Filtration par les sous-groupes de congruence et fonction degré}

Proposition 5. - Supposons $G$ annulé par $p$. Pour $x \in G\left(\theta_{\bar{K}}\right)$, notons $\langle x\rangle$ l'adhérence schématique dans $G_{\vartheta_{\bar{K}}}$ du sous- $\mathbb{F}_{p}$-espace vectoriel engendré par $x$ dans $G\left(\theta_{\bar{K}}\right)$. Alors pour $\lambda \in \Gamma$ vérifiant $0 \leq \lambda \leq \frac{1}{p-1}$,

$$
G_{\text {cong }}^{\lambda}\left(\vartheta_{\bar{K}}\right)=\left\{x \in G\left(\Theta_{\bar{K}}\right) \backslash\{0\} \mid \operatorname{deg}(\langle x\rangle) \geq(p-1) \lambda\right\} \cup\{0\} .
$$

Démonstration. - Soit $x \neq 0$. Posons $E=\langle x\rangle$. On a $x \in G_{\text {cong }}^{\lambda} \Leftrightarrow E_{\text {cong }}^{\lambda} \neq 0$. Mais $E^{D} \simeq C_{u}$ pour un $u \in \theta_{\bar{K}}$ vérifiant $v(u)=\frac{\operatorname{deg}(E)}{p-1}$. Donc, $E_{\text {cong }}^{\lambda} \neq 0$ si et seulement si $\frac{\operatorname{deg}(E)}{p-1} \geq \lambda$. D'où le résultat. 


\subsubsection{Filtration d'Abbes-Saito et dual de la filtration de congruence d'Andreatta-Gasbarri}

Lemme 6. - Soit E un schéma en groupe fini et plat de hauteur 1 sur $\theta_{\bar{K}}$. Soit $\lambda \in \Gamma$ tel que $\lambda>0$. Alors,

$$
E_{\mathrm{AS}}^{\lambda}=\left\{\begin{array}{l}
E \text { si } \lambda \leq \frac{p \operatorname{deg}(E)}{p-1} \\
0 \text { si } \lambda>\frac{p \operatorname{deg}(E)}{p-1} .
\end{array}\right.
$$

Démonstration. - L'algèbre du groupe $E$ est monogène, $E \simeq \operatorname{Spec}\left(\theta_{K}[T] /\left(T^{p}-\delta T\right)\right)$ pour un $\delta \in \Theta_{\bar{K}}$ vérifiant $v(\delta)=\operatorname{deg}(E)$. D'après l'appendice de [14], on a donc

$$
E_{\mathrm{AS}}^{\lambda}=E_{\mathrm{rn}}^{\psi(\lambda)}
$$

où $\psi$ est la fonction de Herbrand de $E$ (cf. l'appendice de [14]), fonction qui se calcule à partir du polygone de Newton du polynôme $T^{p}-\delta T$. On trouve facilement que

$$
\psi(\lambda)=\left\{\begin{array}{c}
\frac{\lambda}{p} \text { si } \lambda \leq \frac{p v(\delta)}{p-1} \\
\lambda-v(\delta) \text { si } \lambda \geq \frac{p v(\delta)}{p-1} .
\end{array}\right.
$$

Le résultat se déduit alors du lemme 5 .

Le résultat qui suit a déjà été démontré par Tian dans [36] (théorème 1.6). Néanmoins, la démonstration de Tian fait intervenir des résolutions des groupes plats finis par des schémas abéliens ainsi que la filtration de Bloch-Kato des cycles évanescents $p$-adiques sur ces schémas abéliens. Nous donnons une démonstration élémentaire de ce résultat.

Proposition 6. - Supposons $G$ annulé par p. Soit $\lambda \in \Gamma$ tel que $\lambda>0$. Alors,

$$
G_{\mathrm{AS}}^{\lambda}=0 \text { si } \lambda>\frac{p}{p-1} \text {. }
$$

Considérons l'accouplement parfait $G\left(\Theta_{\bar{K}}\right) \times G^{D}\left(\theta_{\bar{K}}\right) \rightarrow \mu_{p}(\bar{K})$. Alors, via cet accouplement, si $\lambda<\frac{p}{p-1}$, on $a$

$$
G_{\mathrm{AS}}^{\lambda}\left(\theta_{\bar{K}}\right)^{\perp}=\bigcup_{\lambda^{\prime}>\frac{1}{p-1}-\frac{\lambda}{p}}\left(G^{D}\right)_{\text {cong }}^{\lambda^{\prime}}\left(\theta_{\bar{K}}\right)
$$

Démonstration. - Soit $x \in G^{D}\left(\theta_{\bar{K}}\right) \backslash\{0\}$. Notons $E$ l'adhérence schématique du sousgroupe engendré par $x$ dans $G_{\emptyset_{\bar{K}}}^{D}$. Il y a une factorisation

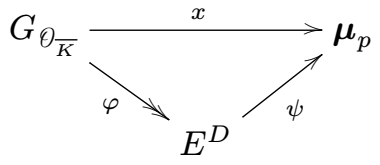

où $\varphi$ est un épimorphisme et $\psi$ est un isomorphisme en fibre générique. La compatibilité de la filtration d'Abbes-Saito aux quotients implique alors que

$$
x \in G_{\mathrm{AS}}^{\lambda}\left(\theta_{\bar{K}}\right)^{\perp} \Longleftrightarrow\left(E^{D}\right)_{\mathrm{AS}}^{\lambda}=0 .
$$

Le résultat se déduit alors du lemme 6 et de la proposition 5 .

4 e SÉRIE - TOME $44-2011$ - No 6 


\section{Sur l'application de Hodge-Tate des groupes $p$-divisibles et des schémas en groupes finis et plats}

Dans cette section nous expliquons plus en détail les théorèmes II.1.1 de [15] et 7 de [16] sur la suite de Hodge-Tate d'un groupe $p$-divisible. Nous expliquons en particulier le lien avec la presque décomposition de Hodge-Tate de Fontaine ([18]), ce qui n'est pas fait dans [15]. Le seul résultat que nous utiliserons dans la suite est le théorème 3 de cette section (qui est le théorème 7 de [16]). Nous en faisons ici beaucoup plus que nécessaire. Cependant, il a semblé adéquat à l'auteur d'expliquer plus en détail ce type de résultat indépendamment de son application à la théorie des sous-groupes canoniques.

Pour montrer le théorème 3 nous utilisons les résultats de [13] concernant les périodes cristallines entières des groupes $p$-divisibles. Notons cependant qu'une modification des arguments de [9] permettrait d'obtenir le théorème 3. Plus précisément, dans [9] ChambertLoir considère le site cristallin relatif de $\operatorname{Spec}\left(\theta_{\bar{K}}\right) / \operatorname{Spec}\left(\theta_{K}\right)$. En remplaçant celui-ci par le site cristallin absolu c'est-à-dire celui associé à $\operatorname{Spec}\left(\theta_{\bar{K}}\right) / \operatorname{Spec}\left(\mathbb{Z}_{p}\right)$ on peut obtenir le théorème 3. Néanmoins l'auteur ignore si la méthode de [9] permet d'obtenir le théorème 2 de cette section. C'est pourquoi nous avons préféré utiliser les méthodes de [13].

Le lecteur effrayé par les considérations de théorie de Hodge $p$-adique pourra aisément admettre le théorème 3 et passer à la section suivante.

\subsection{Relèvement cristallin de l'application de Hodge-Tate}

5.1.1. Relèvement du morphisme $\alpha_{G}$. - Soient $S$ un schéma et $G$ un $S$-schéma en groupes fini localement libre. Comme dans la section 2.1, on note

$$
\alpha_{G}: G \longrightarrow \underline{\omega}_{G^{D}}
$$

l'application universelle de $G$ vers des faisceaux fppf de groupes abéliens de la forme $\underline{\mathcal{M}}$, où $\mathcal{M}$ est un $\theta_{S}$-module quasi-cohérent. Supposons que $p$ soit localement nilpotent sur $S$ et que $S$ soit un $\Sigma$-schéma, où $\Sigma$ est un schéma affine qui est le spectre d'un anneau $p$-adiquement complet sans $p$-torsion. L'idéal $p \theta_{\Sigma}$ de $\theta_{\Sigma}$ est canoniquement muni de puissances divisées. On note CRIS $(S / \Sigma)$ le gros site cristallin fppf de $S / \Sigma$ ([7]). Rappelons ([7] 1.1.4) qu'il y a un morphisme de topos

$$
\left(i_{S / \Sigma}^{*}, i_{S / \Sigma *}\right): \widetilde{S}_{f p p f} \rightarrow(S / \Sigma)_{C R I S}
$$

où si $\mathcal{F}$ est un faisceau fppf sur $S$ et $(U, T, \delta) \in \operatorname{CRIS}(S / \Sigma)$ alors $\Gamma\left((U, T, \delta), i_{S / \Sigma *} \mathcal{F}\right)=\mathcal{F}(U)$. Si $C$ est un $S$-schéma en groupes il définit donc un faisceau cristallin $i_{S / \Sigma *} C$ que nous noterons $\underline{C}$ dans la suite. Posons maintenant

$$
\mathbb{D}(G)=\varepsilon x t^{1}\left(\underline{G}^{D}, \theta_{S / \Sigma}\right)
$$

le cristal de Dieudonné covariant de $G$ ([7] chap. 3). Soit $J_{S / \Sigma}$ l'idéal à puissances divisées canonique de $\theta_{S / \Sigma}$. Il y a une suite exacte

$$
1 \longrightarrow 1+J_{S / \Sigma} \longrightarrow \theta_{S / \Sigma}^{\times} \longrightarrow \mathbb{G}_{m} \longrightarrow 1
$$

qui fournit un morphisme dans la catégorie dérivée des faisceaux de groupes abéliens sur $\operatorname{CRIS}(S / \Sigma), \underline{\mathbb{G}_{m}} \rightarrow\left(1+J_{S / \Sigma}\right)[1]$. On dispose de plus d'un morphisme logarithme ([7], 5.1.7)

$$
\log : 1+J_{S / \Sigma} \longrightarrow J_{S / \Sigma} \text {. }
$$


Poussant l'extension précédente en avant par ce morphisme, on obtient un morphisme dans la catégorie dérivée

$$
\underline{\mathbb{G}_{m}} \longrightarrow J_{S / \Sigma}[1]
$$

Appliquant le foncteur $\operatorname{H} o m\left(\underline{G}^{D},-\right)$ à ce morphisme et composant avec l'inclusion $J_{S / \Sigma} \subset \theta_{S / \Sigma}$, on obtient un morphisme de groupes dans $(S / \Sigma)_{C R I S}$

$$
\alpha_{G}^{\mathrm{cris}}: \underline{G} \longrightarrow \mathbb{D}(G) .
$$

Rappelons ([7], 3.2) que si $\Delta(G)=\tau_{\leq 1} R \mathcal{H} f o m\left(\underline{G}^{D}, \theta_{S / \Sigma}\right) \in \mathbb{D}_{\text {parf }}^{[0,1]}\left(\theta_{S / \Sigma}\right)$ désigne le complexe de Dieudonné covariant de $G$, son évaluation sur l'épaississement tautologique s'insère dans un triangle " filtration de Hodge »

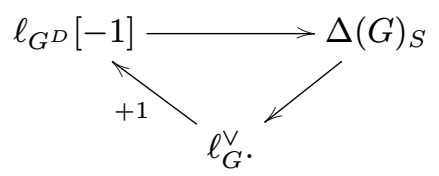

Ce triangle de complexes zariskiens s'identifie à l'évaluation sur l'épaississement tautologique du triangle cristallin

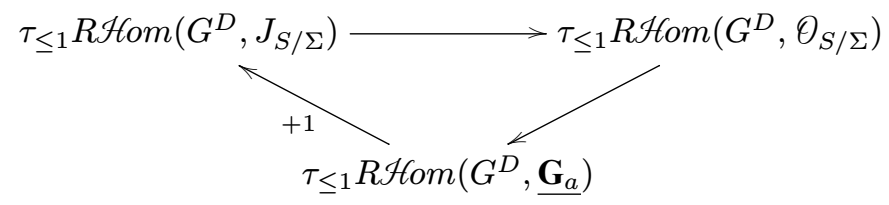

déduit de la suite exacte $0 \rightarrow J_{S / \Sigma} \rightarrow \theta_{S / \Sigma} \rightarrow \underline{\mathbf{G}_{a}} \rightarrow 0$. Le morphisme $\alpha_{G}^{\text {cris }}$ évalué sur l'épaississement tautologique s'insère alors dans un diagramme commutatif

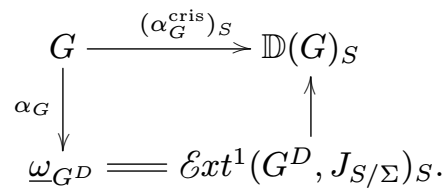

En effet, un tel diagramme est fonctoriel en $G$ et compatible au changement de base. Une section de $G$ étant donnée par un morphisme de schémas en groupes fini et plat $\mathbb{Z} / p^{r} \mathbb{Z} \rightarrow G$ avec $r \gg 0$ il suffit de vérifier que ce diagramme est commutatif lorsque $G=\mathbb{Z} / p^{r} Z$ mais cela ne pose pas de problème. Ainsi, $\alpha_{G}^{\text {cris }}$ est un « relèvement cristallin» de $\alpha_{G}$.

Lorsque $p \vartheta_{S}=0$, l'image de $\alpha_{G}$ est contenue dans $\operatorname{ker}\left(\underline{\omega}_{G^{D}} \stackrel{F-\psi_{G}}{\longrightarrow} \underline{\omega}_{G^{D}}^{(p)}\right)$. Le morphisme $\alpha_{G}^{\text {cris }}$ vérifie le même type d'assertion. Plus précisément, soit $S_{0}$ la réduction modulo $p$ de $S$ et $G_{0}=G \times{ }_{S} S_{0}$. Le site CRIS $\left(S_{0} / \Sigma\right)$ se "plonge» dans le site CRIS $(S / \Sigma)$, l'immersion à puissances divisées $i: S_{0} \hookrightarrow S$ induit un morphisme de topos

$$
\left(i_{\text {cris }}^{*}, i_{\text {cris* }}\right):\left(S_{0} / \Sigma\right)_{C R I S} \longrightarrow(S / \Sigma)_{C R I S} .
$$

Le couple de foncteurs adjoints $\left(i_{\text {cris }}^{*}, i_{\text {cris } *}\right)$ induit de plus une équivalence de catégories entre la catégorie des cristaux de $\theta_{S_{0} / \Sigma}$-modules et celle des cristaux de $\theta_{S / \Sigma}$-modules ([5] IV.1.4.2). Via cette équivalence

$$
\mathbb{D}(G)=i_{\text {cris } *} \mathbb{D}\left(G_{0}\right) \text { et } \mathbb{D}\left(G_{0}\right)=i_{\text {cris }}^{*} \mathbb{D}(G) .
$$


On suppose désormais que $\Sigma=\operatorname{Spec}\left(\mathbb{Z}_{p}\right)$. Le morphisme de Frobenius de $S_{0}$ induit un morphisme Frob $:\left(S_{0}, \Sigma\right) \rightarrow\left(S_{0}, \Sigma\right)$. Si $\mathcal{E}$ est un cristal en $\theta_{S_{0} / \Sigma}$-modules on note $\mathscr{E}^{(p)}=$ Frob $^{*} \mathcal{E}$. Via l'équivalence de catégories précédentes si $\mathcal{E}$ est un cristal en $\theta_{S / \Sigma}$-modules on note $\mathcal{E}^{(p)}=i_{\text {cris* }}\left(\left(i_{\text {cris }}^{*} \mathcal{E}\right)^{(p)}\right)$. Concrètement, si $(U, T, \delta) \in \operatorname{CRIS}(S / \Sigma)$, $U_{0}=U \times_{S} S_{0}$, s'il existe $\varphi: T \rightarrow T$ relevant le Frobenius de $U_{0}$,

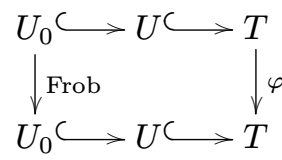

et $\varphi$ commute aux puissances divisées de l'idéal de $U_{0}$ dans $T$ associées à $\delta$, alors

$$
\left(\mathcal{E}^{(p)}\right)_{(U, T, \delta)}=\varphi^{*}\left(\mathcal{E}_{(U, T, \delta)}\right)
$$

où si $\mathcal{F} \in(S / \Sigma)_{C R I S}$ on note $\mathcal{F}_{(U, T, \delta)}$ le faisceau associé sur le petit site fppf de $T$. Le morphisme de Frobenius $F: G_{0} \rightarrow G_{0}^{(p)}$ induit un morphisme

$$
V=F_{*}: \mathbb{D}\left(G_{0}\right) \longrightarrow \mathbb{D}\left(G_{0}^{(p)}\right)=\mathbb{D}\left(G_{0}\right)^{(p)} .
$$

Il induit donc un morphisme noté de la même façon

$$
V: \mathbb{D}(G) \longrightarrow \mathbb{D}(G)^{(p)}
$$

Soit maintenant $((U, T, \delta), \varphi)$ comme précédemment. Notons $\pi$ la projection du petit topos fppf de $T$ sur son topos zariskien. Soit

$$
\mathcal{E}=\pi_{*} \mathbb{D}(G)_{(U, T, \delta)},
$$

un $\theta_{T}$-module cohérent tel que le morphisme d'adjonction

$$
\underline{\mathcal{E}}:=\pi^{*} \mathcal{E} \longrightarrow \mathbb{D}(G)_{(U, T, \delta)}
$$

soit un isomorphisme. Si $\kappa: U \hookrightarrow T$, le morphisme de faisceaux induit par $\alpha_{G}^{\text {cris }}$,

$$
\left(\alpha_{G}^{\text {cris }}\right)_{(U, T, \delta)}: \kappa_{*}\left(G \times_{S} U\right) \longrightarrow \underline{\mathcal{E}},
$$

vérifie via la formule (2)

$$
\operatorname{Im}\left(\left(a_{G}^{\text {cris }}\right)_{(U, T, \delta)}\right) \subset\{x \in \underline{\mathcal{E}} \mid V(x)=x \otimes 1\},
$$

où $V: \mathcal{E} \rightarrow \varphi^{*} \mathcal{E}$. Cette formule relève alors la formule $\operatorname{Im}\left(\alpha_{G_{0}}\right) \subset \operatorname{ker}\left(\underline{\omega}_{G_{0}^{D}} \stackrel{F-\psi_{G}}{\longrightarrow} \underline{\omega}_{G_{0}^{D}}^{(p)}\right)$.

Soit maintenant $I$ l'idéal de $U$ dans $T$. D'après les considération précédentes sur la filtration de Hodge il y a un morphisme de $\theta_{U}$-modules $\omega_{G^{D} \times_{S} U}=\omega_{G^{D}} \otimes_{\vartheta_{S}} \theta_{U} \rightarrow \mathcal{E} / \mathcal{J} \mathcal{E}$. Notons Fil $\mathscr{E}$ le sous- $\theta_{T}$-module de $\mathscr{E}$ tel que $\mathscr{J} \mathscr{E} \subset$ Fil $\mathscr{E}$ et $\mathscr{E} /$ Fil $\mathscr{E}=$ $\operatorname{Im}\left(\omega_{G^{D}} \otimes_{\vartheta_{S}} \theta_{U} \rightarrow \mathcal{E} / \mathcal{J} \mathcal{E}\right)$. On obtient au final que l'application des périodes $\alpha_{G}^{\text {cris }}$ évaluée $\operatorname{sur}(U, T, \delta)$ vérifie

$$
\operatorname{Im}\left(\left(\alpha_{G}^{\text {cris }}\right)_{(U, T, \delta)}\right) \subset\{x \in \underline{\text { Fil } \mathcal{E}} \mid V(x)=x \otimes 1\} .
$$


5.1.2. Relèvement de l'application de Hodge-Tate. - Soit maintenant $G$ un groupe plat fini $\operatorname{sur} \theta_{K}$. On note $S=\operatorname{Spec}\left(\theta_{K}\right), S_{0}=\operatorname{Spec}\left(\theta_{K} / p \theta_{K}\right), G_{0}=G \times_{S} S_{0}, \bar{S}=\operatorname{Spec}\left(\theta_{\widehat{K}}\right)$, $\bar{S}_{0}=\operatorname{Spec}\left(\Theta_{\bar{K}} / p \Theta_{\bar{K}}\right)$ et $\Sigma=\operatorname{Spec}\left(\mathbb{Z}_{p}\right)$. Rappelons que, d'après Fontaine, puisque le morphisme de Frobenius de $\theta_{\bar{K}} / p \theta_{\bar{K}}$ est surjectif, la catégorie CRIS $\left(\bar{S}_{0} / \Sigma\right)$ possède un indobjet initial, l'épaississement $p$-adique

$$
A_{\text {cris }} \stackrel{\theta}{\longrightarrow} \theta_{\widehat{\bar{K}}} \longrightarrow \theta_{\bar{K}} / p \theta_{\bar{K}} .
$$

Si $\mathscr{R}=\lim _{\overleftarrow{N}} \theta_{\bar{K}} / p \theta_{\bar{K}}$ désigne le perfectisé de $\theta_{\bar{K}} / p \theta_{\bar{K}}$, il y a une application surjective $\theta: W(\mathscr{R}) \rightarrow \oslash_{\widehat{K}}$ et l'anneau de Fontaine $A_{\text {cris }}$ est le complété $p$-adique de l'enveloppe à puissances divisées de $W(\mathcal{R})$ relativement à $\operatorname{ker}(\theta)$ ([19]). Il est muni d'un Frobenius cristallin $\varphi$ et d'une action de $\operatorname{Gal}(\bar{K} \mid K)$. Soit alors

$$
\begin{aligned}
E & =H^{0}\left(\bar{S}_{0} / \Sigma, \mathbb{D}\left(G_{\bar{S}_{0}}\right)\right) \\
& =\overleftarrow{n \geq 0}_{\lim _{n \geq}} \Gamma\left(\operatorname{Spec}\left(\theta_{\bar{K}} / p \vartheta_{\bar{K}}\right) \hookrightarrow \operatorname{Spec}\left(A_{\text {cris }} / p^{n} A_{\text {cris }}\right), \mathbb{D}\left(G_{0}\right)\right) .
\end{aligned}
$$

C'est un $A_{\text {cris }}$-module de présentation finie annulé par une puissance de $p$ et muni d'un Verschiebung, un morphisme $A_{\text {cris }}$-linéaire

$$
V: E \longrightarrow E \underset{A_{\text {cris }, \varphi}}{\otimes} A_{\text {cris }}
$$

Si $G$ est un groupe de Barstotti-Tate tronqué d'échelon $n$ et de hauteur $h$, c'est un $A_{\text {cris }} / p^{n} A_{\text {cris }}$-module libre de rang $h$. L'application des périodes précédentes définit un morphisme $\operatorname{Gal}(\bar{K} \mid K)$-équivariant

$$
G\left(\vartheta_{\bar{K}} / p \vartheta_{\bar{K}}\right) \longrightarrow\{x \in E \mid V(x)=x \otimes 1\} .
$$

Soit maintenant

$$
M=\mathbb{D}\left(G_{0}\right)_{S_{0} \hookrightarrow S},
$$

un $\theta_{K}$-module de présentation finie de torsion. D'après la propriété de cristal on dispose d'une identification canonique

$$
E \underset{A_{\text {cris }, \theta}}{\otimes} \theta_{\widehat{K}}=M \otimes_{\vartheta_{K}} \theta_{\widehat{K}}
$$

car le membre de gauche s'identifie à $\mathbb{D}\left(G_{0}\right)_{\bar{S}_{0} \hookrightarrow \bar{S}}$. Remarquons maintenant que $H^{-1}\left(\ell_{G}\right)=0$ et $H^{-1}\left(\ell_{G^{D}}\right)=0$. Le triangle filtration de Hodge (1) insère donc $M$ dans une suite exacte de $\theta_{K}$-modules de torsion

$$
0 \longrightarrow \omega_{G^{D}} \longrightarrow M \longrightarrow \omega_{G}^{\vee} \longrightarrow 0
$$

où $\omega_{G}^{\vee}:=\operatorname{Hom}_{\vartheta_{K}}\left(\omega_{G}, K / \vartheta_{K}\right)$ désigne le dual de Pontryagin du $\vartheta_{K}$-module de torsion $\omega_{G}$. Notons alors Fil $M=\omega_{G^{D}}$, sa filtration de Hodge. Lorsque $G$ est un groupe de Barsotti-Tate tronqué d'échelon $n$, de hauteur $h$ et de dimension $d, M$ et $\omega_{G^{D}}$ sont des $\vartheta_{K} / p^{n} \vartheta_{K}$-modules libres de rang $h$ et $h-d$. Dans ce cas là, Fil $M$ est un $\theta_{K} / p^{n} \theta_{K}$-module facteur direct dans $M$. 
Via l'isomorphisme $E \otimes_{A_{\text {cris }}, \theta} \theta_{\widehat{\bar{K}}} \stackrel{\sim}{\rightarrow} M \otimes \theta_{\widehat{\bar{K}}}$, notons Fil $E$ l'image réciproque par $\theta$ de Fil $M \otimes \theta_{\widehat{K}}$. On a donc

$$
\text { Fil }^{1} A_{\text {cris }} E \subset \text { Fil } E \subset E \text { et Fil } E / \text { Fil }^{1} A_{\text {cris }} E=\text { Fil } M \otimes \theta_{\widehat{K}} .
$$

La composée de l'application des périodes (3) avec l'application de réduction $G\left(\theta_{\bar{K}}\right) \rightarrow G\left(\theta_{\bar{K}} / p \Theta_{\bar{K}}\right)$ définit un morphisme $\alpha_{G}^{\text {cris }}$ s'insérant dans un diagramme

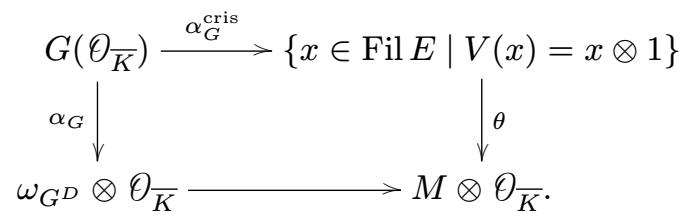

\subsection{Un isomorphisme des périodes entier pour les groupes $p$-divisibles}

On reprend les notations de la section précédente. Soit maintenant $H$ un groupe $p$-divisible sur $\vartheta_{K}$. Notons $H_{0}=H_{S_{0}}$ la réduction modulo $p$ de $H$. Soit

$$
\mathbb{D}\left(H_{0}\right)=\mathcal{E} t^{1}\left(\underline{H_{0}^{D}}, \theta_{S / \Sigma}\right)
$$

son cristal de Dieudonné covariant ([7], 3.3). Soit

$$
E=H^{0}\left(\bar{S}_{0} / \Sigma, \mathbb{D}\left(H_{0}\right)\right),
$$

un $A_{\text {cris }}$-module libre de rang la hauteur de $H$, muni d'un Verschiebung $V: E \rightarrow E \underset{A_{\text {cris }}, \varphi}{\otimes} A_{\text {cris }}$ induit par $F: H_{0} \rightarrow H_{0}^{(p)}$ et d'un Frobenius $F: E \underset{A_{\text {cris }, \varphi}}{\otimes} A_{\text {cris }} \rightarrow E$ induit par $V: H_{0}^{(p)} \rightarrow H_{0}$

Décrivons plus précisément ce module $E$. Il y a un morphisme surjectif

$$
\begin{aligned}
\mathscr{R}=\left\{\left(x_{i}\right)_{i \in \mathbb{N}} \mid x_{i} \in \theta_{\bar{K}} / p \vartheta_{\bar{K}}, x_{i+1}^{p}=x_{i}\right\} & \longrightarrow \vartheta_{\bar{K}} / p \vartheta_{\bar{K}} \\
\left(x_{i}\right)_{i \geq 0} & \longmapsto x_{0} .
\end{aligned}
$$

L'application de réduction modulo $p$ induit une bijection

$$
\left\{\left(x^{(i)}\right)_{i \in \mathbb{N}} \mid x^{(i)} \in \Theta_{\widehat{K}},\left(x^{(i+1)}\right)^{p}=x^{(i)}\right\} \stackrel{\sim}{\longrightarrow} \mathcal{R} .
$$

Via cette bijection, la fonction $v: \mathscr{R} \rightarrow \mathbb{R} \cup\{+\infty\}$ qui à $\left(x^{(i)}\right)_{i \geq 0}$ associe $v\left(x^{(0)}\right)$ définit une valuation sur $\mathscr{R}$. L'anneau $\mathscr{R}$ est alors un anneau de valuation complet parfait de caractéristique $p$ et $\mathfrak{a}:=\operatorname{ker}\left(\mathscr{R} \rightarrow \Theta_{\bar{K}} / p \theta_{\bar{K}}\right)=\{x \in \mathscr{R} \mid v(x) \geq 1\}$, i.e.

$$
\mathscr{R} / \mathfrak{a} \stackrel{\sim}{\longrightarrow} \theta_{\bar{K}} / p \theta_{\bar{K}} \text {. }
$$

Puisque $\mathscr{R}$ est a-adiquement complet, le groupe $p$-divisible $H_{\bar{S}_{0}}$ sur $\operatorname{Spec}\left(\theta_{\bar{K}} / p \theta_{\bar{K}}\right)$ se relève en un groupe $p$-divisible $\widetilde{H}_{0} \operatorname{sur} \operatorname{Spf}(\mathscr{R})$ que l'on voit encore comme un groupe $p$-divisible $\widetilde{H}_{0}$ $\operatorname{sur} \operatorname{Spec}(\mathscr{R})([31], 4.16)$. Soit alors

$$
N=\mathbb{D}\left(\widetilde{H}_{0}\right)_{W(\mathscr{R}) \longrightarrow \mathscr{R}}
$$

un $W(\mathscr{R})$-module libre muni de deux morphismes $F: N \rightarrow N$, $\sigma$-linéaire, et $V: N \rightarrow N$, $\sigma^{-1}$-linéaire. $\mathrm{Si}$

$$
M\left(\widetilde{H}_{0}\right)=\operatorname{Hom}\left(\widetilde{H}_{0}^{D}, C W\right)
$$


désigne le module de Dieudonné covariant «classique» de $\widetilde{H}_{0}$ ([17] chap. III), il y a un isomorphisme canonique de $W(\mathscr{R})[F, V]$-modules ([7] 4.2.15, [6])

$$
M\left(\widetilde{H}_{0}\right)^{\sigma} \simeq N .
$$

Le morphisme de CRIS $\left(\bar{S}_{0} / \Sigma\right)$

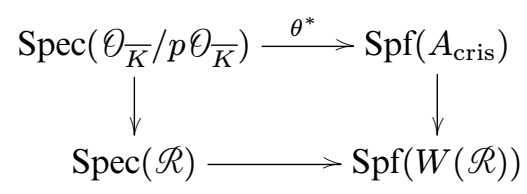

couplé à la propriété de cristal induit alors un isomorphisme canonique compatible aux opérateurs $F$ et $V$

$$
E \simeq N \otimes_{W(\mathscr{R})} A_{\text {cris }} .
$$

Supposons momentanément que $K$ soit de valuation discrète à corps résiduel parfait. Notons alors $H_{k_{K}}$ la réduction sur le corps résiduel de $K$ de $H$. Via la section de Teichmüller $\tau: k \hookrightarrow \vartheta_{K} / p \vartheta_{K}$, par rigidité des quasi-isogénies, il existe une unique quasi-isogénie

$$
\rho: H_{k_{K}} \otimes_{k_{K}, \tau} \theta_{K} / p \theta_{K} \longrightarrow H_{0}
$$

relevant l'identité de $H_{k_{K}}$. Soit

$$
M\left(H_{k_{K}}\right)=\operatorname{Hom}\left(H_{k_{K}}^{D}, C W\right)
$$

le module de Dieudonné «classique» de $H_{k_{K}}$. Il y a un isomorphisme ([7] 4.2.14)

$$
\mathbb{D}\left(H_{k_{K}}\right)_{W\left(k_{K}\right) \rightarrow k_{K}} \simeq M\left(H_{k_{K}}\right)^{\sigma}
$$

et donc un isomorphisme

$$
\rho_{*}: M\left(H_{k_{K}}\right)^{\sigma}\left[\frac{1}{p}\right] \otimes_{W\left(k_{K}\right)_{\mathbb{Q}}} B_{\text {cris }}^{+} \stackrel{\sim}{\longrightarrow} E\left[\frac{1}{p}\right]
$$

où l'on note $B_{\text {cris }}^{+}:=A_{\text {cris }}\left[\frac{1}{p}\right]$. Néanmoins, nous nous intéressons ici à des isomorphismes de périodes entiers. C'est pourquoi nous n'utiliserons pas cette identification lorsque $K$ est de valuation discrète à corps résiduel parfait.

Considérons maintenant

$$
M=\mathbb{D}(H)_{S_{0} \hookrightarrow S},
$$

un $\theta_{K}$-module libre de rang la hauteur de $H$. Il est muni d'une filtration de Hodge Fil $M=\omega_{H^{D}}$ qui s'insère dans la suite exacte

$$
0 \longrightarrow \omega_{H^{D}} \longrightarrow M \longrightarrow \omega_{H}^{*} \longrightarrow 0 .
$$

Il y a un isomorphisme

$$
E \underset{A_{\text {cris }}, \theta}{\otimes} \theta_{\widehat{K}} \simeq M \otimes_{\vartheta_{K}} \theta_{\widehat{K}} \text {. }
$$

Soit alors Fil $E \subset E$ l'image réciproque via $\theta$ de la filtration de Hodge,

$$
\text { Fil }^{1} A_{\text {cris }} E \subset \text { Fil } E \subset E \text { et Fil } E / \text { Fil }^{1} A_{\text {cris }} E=\text { Fil } M \otimes \theta_{\widehat{K}} \text {. }
$$

Les applications de périodes $\alpha_{H\left[p^{n}\right]}^{\text {cris }}$ sont compatibles lorsque $n$ varie et fournissent une application des périodes

$$
\alpha_{H}^{\text {cris }}: T_{p}(H) \longrightarrow \text { Fil } E^{\varphi=p}
$$

$4^{\text {e }}$ SÉRIE - TOME $44-2011-$ No $^{\circ}$ 
où $\varphi: E \rightarrow E$ est le morphisme $\left(A_{\text {cris }}, \varphi\right)$-linéaire défini par $\varphi(x)=F(x \otimes 1)$. Cette application relève l'application de Hodge-Tate de $H, \alpha_{H}: T_{p}(H) \rightarrow \omega_{H^{D}} \otimes \theta_{\widehat{\bar{K}}}$ au sens où la composée

$$
T_{p}(H) \stackrel{\alpha_{H}^{\text {cris }}}{\longrightarrow} \text { Fil } E^{\varphi=p} \stackrel{\theta}{\longrightarrow} \omega_{H^{D}} \otimes \theta_{\widehat{K}}
$$

coïncide avec $\alpha_{H}$. Rappelons que $t \in \mathrm{Fil}^{1} A_{\text {cris }}$ désigne une période du groupe multiplicatif (cf. [19]). On a alors le théorème suivant.

ThÉORÈme 1 ([13] théorèmes 7 et $5 *$ ). - Supposons $p \neq 2$. Soit $H$ un groupe $p$-divisible sur $\theta_{K}$.

1. L'application des périodes précédente induit un isomorphisme

$$
T_{p}(H) \stackrel{\sim}{\longrightarrow} \text { Fil } E^{\varphi=p}
$$

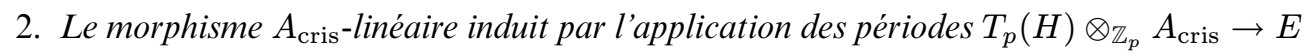
est injectif de conoyau annulé par $t$.

3. Munissons $E$ de la filtration décroissante définie par $\mathrm{Fil}^{-1} E=E, \mathrm{Fil}^{0} E=$ Fil $E$ et pour $i \geq 1, \mathrm{Fil}^{i} E=\mathrm{Fil}^{i+1} A_{\text {cris }} . E+\mathrm{Fil}^{i} A_{\text {cris }}$. Fil $E$. Munissons $T_{p}(H) \otimes A_{\text {cris }}$ de la filtration $\operatorname{Fil}^{i}\left(T_{p}(H) \otimes A_{\text {cris }}\right)=T_{p}(H) \otimes$ Fil $^{i} A_{\text {cris. }}$ Posons $\operatorname{deg}(t)=1$. Les inclusions de $A_{\text {cris }}$-modules filtrés

$$
t E \subset T_{p}(H) \otimes_{\mathbb{Z}_{p}} A_{\text {cris }} \subset E
$$

sont alors strictement compatibles aux filtrations.

Remarque 2. - Dans [13] le théorème précédent est démontré lorsque $K$ est de valuation discrète. Néanmoins, les résultats de [13] énoncés précédemment s'étendent au cas que nous considérons, la propriété essentielle étant que le Frobenius de $\oslash_{\bar{K}} / p \theta_{\bar{K}}$ est surjectif ce qui est vérifié dans un contexte plus général que celui de [13] (cf. la discussion avant l'énoncé du théorème $5^{*}$ de [13]).

- Tel qu'énoncé dans [13], le théorème fait intervenir le complété de $A_{\text {cris }}$ relativement à sa filtration à puissances divisées, i.e. Faltings travaille avec le site cristallin nilpotent au lieu du site cristallin. Dans la suite de cet article nous n'utiliserons en fait que les gradués de $A_{\text {cris, }}$ c'est pourquoi cela ne pose pas de problème. Néanmoins, le lecteur pourra vérifier que les preuves de [13] montrent le résultat énoncé précédemment sans passer au complété relativement à la PD filtration.

- Lorsque $p=2$ on peut montrer un résultat moins précis : l'application $T_{p}(H) \rightarrow$ Fil $E^{\varphi=p}$ est injective de conoyau annulé par 2 . Le reste de l'énoncé est valable sans restriction.

- L'inclusion $t E \subset T_{p}(H) \otimes A_{\text {cris }}$ induit par dualisation un morphisme $t T_{p}(H)^{*} \otimes A_{\text {cris }} \rightarrow$ $E^{*}$. Il y a une identification canonique $t T_{p}(H)^{*}=T_{p}(H)^{*}(1)=T_{p}\left(H^{D}\right)$. De plus, $E^{*}$ s'identifie à l'évaluation du cristal de Dieudonné de $H^{D}$ ([7] 5.3). Via ces identifications, le morphisme $T_{p}\left(H^{D}\right) \rightarrow E^{*}$ est $a_{H^{D}}^{\text {cris }}$. 


\subsection{Sur la suite de Hodge-Tate entière d'un groupe $p$-divisible}

5.3.1. Structure de $\operatorname{gr}^{1} A_{\text {cris }}$. - Notons $A_{\text {cris }}\left(\theta_{\bar{K}}\right)$ pour l'anneau noté précédemment $A_{\text {cris }}$. Le morphisme naturel de $\operatorname{gr}^{0} A_{\text {cris }}\left(\theta_{\bar{K}}\right)=\theta_{\widehat{K}}$-algèbres graduées à puissances divisées

$$
\operatorname{gr}^{\bullet} A_{\text {cris }}\left(\overline{\mathbb{Z}}_{p}\right) \underset{\operatorname{gr}^{0} A_{\text {cris }}\left(\overline{\mathbb{Z}}_{p}\right)}{\otimes} \operatorname{gr}^{0} A_{\text {cris }}\left(\theta_{\bar{K}}\right) \longrightarrow \operatorname{gr} A_{\text {cris }}\left(\theta_{\bar{K}}\right)
$$

est un isomorphisme. Le $\overline{\mathbb{Z}}_{p}$-module des différentielles de Kähler $\Omega_{\overline{\mathbb{Z}}_{p} / \mathbb{Z}_{p}}$ est $p$-divisible de torsion. Fontaine construit dans la section 1.4 de [19] un isomorphisme canonique $\operatorname{Gal}\left(\overline{\mathbb{Q}}_{p} \mid \mathbb{Q}_{p}\right)$-équivariant de $\widehat{\overline{\mathbb{Z}}}_{p}$-modules

$$
T_{p}\left(\Omega_{\overline{\mathbb{Z}}_{p} / \mathbb{Z}_{p}}\right) \stackrel{\sim}{\longrightarrow} \operatorname{gr}^{1} A_{\text {cris }}\left(\overline{\mathbb{Z}}_{p}\right) .
$$

L'application des périodes

$$
\mathbb{Z}_{p}(1)=T_{p}\left(\boldsymbol{\mu}_{p^{\infty}}\right) \stackrel{\alpha_{\mu_{p} \infty}^{\text {cris }}}{\longrightarrow} \operatorname{Fil}^{1} A_{\text {cris }}\left(\overline{\mathbb{Z}}_{p}\right)
$$

a pour image $\mathbb{Z}_{p} . t$. De plus, la composée

$$
T_{p}\left(\boldsymbol{\mu}_{p^{\infty}}\right) \stackrel{\alpha_{\mu_{p} \infty}^{\text {cris }}}{\longrightarrow} \operatorname{Fil}^{1} A_{\text {cris }}\left(\overline{\mathbb{Z}}_{p}\right) \longrightarrow \operatorname{gr}^{1} A_{\text {cris }}\left(\overline{\mathbb{Z}}_{p}\right) \simeq T_{p}\left(\Omega_{\overline{\mathbb{Z}}_{p} / \mathbb{Z}_{p}}\right)
$$

est l'application

$$
\begin{aligned}
\text { dlog }: \mathbb{Z}_{p}(1) & \longrightarrow T_{p}\left(\Omega_{\overline{\mathbb{Z}}_{p} / \mathbb{Z}_{p}}\right) \\
\left(\epsilon_{n}\right)_{n \geq 1} & \mapsto\left(\epsilon_{n}^{-1} d \epsilon_{n}\right)_{n \geq 1}
\end{aligned}
$$

où $\epsilon_{n} \in \overline{\mathbb{Z}}_{p}$ est une racine $p^{n}$-ième de l'unité et $\epsilon_{n+1}^{p}=\epsilon_{n}$. D'après le théorème 1 de [18], celle-ci induit une injection de $\widehat{\mathbb{Z}}_{p}$-module libre de rang 1

$$
\widehat{\overline{\mathbb{Z}}}_{p}(1) \hookrightarrow T_{p}\left(\Omega_{\overline{\mathbb{Z}}_{p} / \mathbb{Z}_{p}}\right)
$$

de conoyau annulé par $p^{\frac{1}{p-1}}$. On en déduit que l'application

$$
\theta_{\widehat{K}}(1) \longrightarrow T_{p}\left(\Omega_{\overline{\mathbb{Z}}_{p} / \mathbb{Z}_{p}}\right) \otimes_{\overline{\mathbb{Z}}_{p}} \theta_{\widehat{\bar{K}}} \simeq \operatorname{gr}^{1} A_{\text {cris }}\left(\theta_{\bar{K}}\right)
$$

est injective de conoyau annulé par $p^{\frac{1}{p-1}}$.

5.3.2. La cohomologie de la suite de Hodge-Tate entière est annulée par $p^{\frac{1}{p-1}}$. - Voici maintenant le théorème II.1.1 de [15].

ThÉORÈme 2 ([15]). - Soit $H$ un groupe $p$-divisible sur $\vartheta_{K}$. La cohomologie de la suite de Hodge-Tate de $H$

$$
0 \longrightarrow \omega_{H}^{*} \otimes \theta_{\widehat{K}}(1) \stackrel{{ }^{t}\left(\alpha_{H^{D}} \otimes 1\right)(1)}{\longrightarrow} T_{p}(H) \otimes \theta_{\widehat{K}} \stackrel{\alpha_{H} \otimes 1}{\longrightarrow} \omega_{H^{D}} \otimes \theta_{\widehat{K}} \longrightarrow 0
$$

est annulée par tout élément de $\theta_{\widehat{\bar{K}}}$ de valuation supérieure ou égale à $\frac{1}{p-1}$. En particulier l'application de gauche est injective et la composée des deux applications est nulle (i.e. la suite est bien un complexe). 
Démonstration. - Il y a un diagramme de $\theta_{\widehat{K}}$-modules

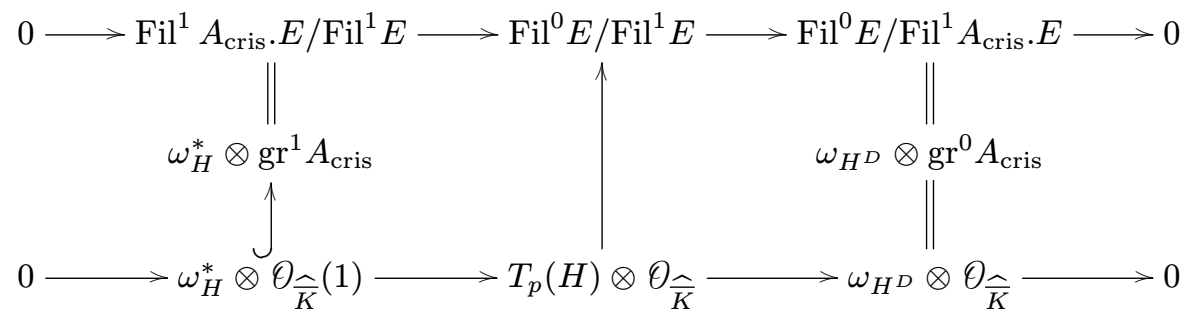

où

- la suite horizontale du haut est exacte,

- la suite horizontale du bas est la suite de Hodge-Tate,

- l'application verticale de gauche est induite par l'application (4) de la sous-section précédente,

- l'application verticale du milieu est induite par l'application des périodes $T_{p}(H) \otimes A_{\text {cris }} \rightarrow$ Fil $E$.

On sait que le conoyau de l'application verticale de gauche est annulé par $p^{\frac{1}{p-1}}$. Il suffit donc de voir qu'il en est de même pour l'application verticale du milieu. Or, il y a un diagramme commutatif

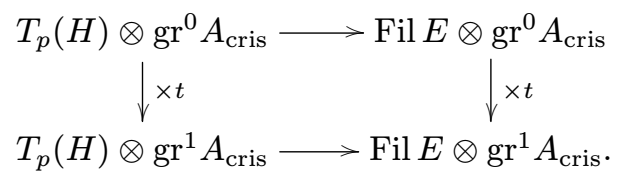

La stricte compatibilité aux filtrations de l'inclusion $t E \subset T_{p}(H) \otimes A_{\text {cris }}$ implique que dans le diagramme précédent l'image de l'application verticale de droite est contenue dans l'image de l'application horizontale du bas. On conclut aisément.

\subsection{Application au conoyau de l'application de Hodge-Tate}

Du théorème 2 précédent on déduit le théorème 6 de [16], auquel on renvoie pour la preuve.

ThÉORÈme 3 ([16]). - Soit $G$ un schéma en groupes fini et plat sur $\theta_{K}$. Le conoyau de l'application de Hodge-Tate de $G, \alpha_{G} \otimes 1: G\left(\theta_{\bar{K}}\right) \otimes \vartheta_{\bar{K}} \rightarrow \omega_{G^{D}} \otimes \vartheta_{\bar{K}}$, est annulé par tout élément de $\theta_{\bar{K}}$ de valuation supérieure ou égale à $\frac{1}{p-1}$.

\subsection{Lien avec la presque décomposition de Hodge-Tate de Fontaine}

Dans cette section on suppose que $K$ est de valuation discrète à corps résiduel parfait. On note $K_{0} \subset K$ le corps des fractions des vecteurs de Witt à coefficients dans le corps résiduel de $K$. 
5.5.1. L'anneau $A_{\mathrm{cris}, K}$. - Reprenons les notations de la section 5.1. Nous avons considéré précédemment les sites cristallins $\operatorname{CRIS}\left(S_{0} / \Sigma\right)$ et $\operatorname{CRIS}\left(\bar{S}_{0} / \Sigma\right)$ avec $S_{0}=\operatorname{Spec}\left(\theta_{K} / p \theta_{K}\right)$, $\bar{S}_{0}=\operatorname{Spec}\left(\theta_{\bar{K}} / p \theta_{\bar{K}}\right)$ et $\Sigma=\operatorname{Spec}\left(\mathbb{Z}_{p}\right)$. Soit maintenant $\Sigma_{K}=\operatorname{Spec}\left(\theta_{K}\right)$. Le site $\operatorname{CRIS}\left(\bar{S}_{0} / \Sigma_{K}\right)$ possède un objet initial donné par l'épaississement

$$
A_{\text {cris }, K} \stackrel{\theta}{\longrightarrow} \theta_{\widehat{\bar{K}}} \longrightarrow \theta_{\bar{K}} / p \theta_{\bar{K}}
$$

où, si $\theta: W(\mathscr{R}) \rightarrow \theta_{\widehat{K}}, A_{\text {cris, } K}$ est le complété $p$-adique de l'enveloppe à puissances divisées du noyau de l'application

$$
\theta \otimes 1: W(\mathscr{R}) \otimes_{\vartheta_{K_{0}}} \theta_{K} \longrightarrow \theta_{\widehat{K}}
$$

et on note encore $\theta: A_{\text {cris }, K} \rightarrow \theta_{\widehat{K}}$ l'application induite par $\theta \otimes 1$. D'après la section 1.4 de [19] il y a un isomorphisme

$$
T_{p}\left(\Omega_{\vartheta_{\bar{K}} / \vartheta_{K}}\right) \simeq \operatorname{gr}^{1} A_{\text {cris }, K}
$$

Il y a un morphisme naturel de $\theta_{\widehat{K}}$-modules $\operatorname{gr}^{1} A_{\text {cris }} \rightarrow \operatorname{gr}^{1} A_{\text {cris }, K}$ et le diagramme suivant est commutatif

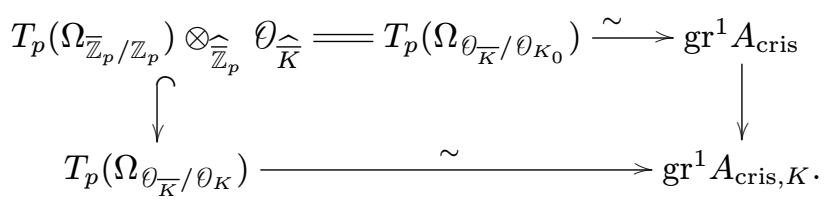

De ce diagramme on déduit que le morphisme $\mathrm{gr}^{1} A_{\text {cris }} \rightarrow \mathrm{gr}^{1} A_{\text {cris }, K}$ est injectif de conoyau annulé par $v\left(\mathscr{D}_{K / K_{0}}\right)$ où $\mathscr{D}_{K / K_{0}}$ désigne la différence de $K \mid K_{0}$. En particulier l'annulateur de l'image de $t$ dans $\operatorname{gr}^{1} A_{\text {cris }, K}$ est de valuation $\frac{1}{p-1}+v\left(\mathscr{D}_{K / K_{0}}\right)$.

5.5.2. Presque décomposition de Hodge-Tate. - Soit $H$ un groupe $p$-divisible sur $\theta_{K}$. Rappelons que l'on note $H_{0}$ sa réduction modulo $p$ et $\mathbb{D}\left(H_{0}\right)$ son cristal de Dieudonné covariant. Soit

$$
E_{K}=H^{0}\left(\bar{S}_{0} / \Sigma_{K}, \mathbb{D}\left(H_{0}\right)\right)
$$

l'évaluation de ce cristal sur l'épaississement $A_{\text {cris }, K} \stackrel{\theta}{\rightarrow} \theta_{\widehat{\bar{K}}} \rightarrow \theta_{\bar{K}} / p \theta_{\bar{K}}$. C'est un $A_{\text {cris, } K}$-module libre de rang la hauteur de $H$. Rappelons que l'on note $E=H^{0}\left(\bar{S}_{0} / \Sigma, \mathbb{D}\left(H_{0}\right)\right)$. D'après la propriété de cristal, il y a un isomorphisme canonique

$$
E_{K} \simeq E \underset{A_{\text {cris }}}{\otimes} A_{\text {cris }, K}
$$

Par application de $(-) \otimes_{A_{\text {cris }}} A_{\text {cris }, K}$ aux inclusions $t E \subset T_{p}(H) \otimes A_{\text {cris }} \subset E$, on obtient que l'application des périodes $T_{p}(H) \rightarrow E_{K}$ induit une injection $T_{p}(H) \otimes A_{\text {cris }, K} \subset E_{K}$ de conoyau annulé par $t$, c'est-à-dire

$$
t E_{K} \subset T_{p}(H) \otimes A_{\text {cris }, K} \subset E_{K} .
$$

Comme pour $E, E_{K}$ est muni d'une filtration Fil $E_{K}$ qui induit une filtration $\left(\mathrm{Fil}^{i} E_{K}\right)_{i \geq-1}$ de $E_{K}$ avec Fil $^{0} E_{K}=$ Fil $E_{K}$. Le sous-module Fil $E_{K}$ de $E_{K}$ est l'image de Fil $E \otimes A_{\text {cris }, K}$ dans $E_{K}=E \otimes_{A_{\text {cris }}} A_{\text {cris }, K}$. L'application des périodes $T_{p}(H) \rightarrow E_{K}$ est à valeurs dans Fil $E_{K}$. On en déduit que les inclusions $t E_{K} \subset T_{p}(H) \otimes_{\mathbb{Z}_{p}} A_{\text {cris }, K} \subset E_{K}$ sont compatibles aux filtrations. Comme dans la preuve du théorème 1 (cf. [13]), la compatibilité de l'application des périodes à la dualité de Cartier et la compatibilité aux filtrations des 
inclusions du type précédent associées à $H^{D}$ impliquent que les inclusions $t E_{K} \subset T_{p}(H) \otimes$ $A_{\text {cris }, K} \subset E_{K}$ sont strictement compatibles aux filtrations.

Le point nouveau par rapport au cas $\Sigma=\operatorname{Spec}\left(\mathbb{Z}_{p}\right)$ est maintenant le suivant. Soit $M=\mathbb{D}\left(H_{0}\right)_{S_{0} \hookrightarrow S}$ muni de sa filtration de Hodge $0 \rightarrow \omega_{H}^{*} \rightarrow M \rightarrow \omega_{H^{D}} \rightarrow 0$. Il y a un morphisme d'épaississement dans $\operatorname{CRIS}\left(S_{0} / \Sigma_{K}\right)$

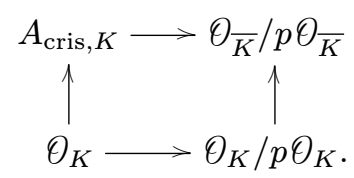

Celui-ci induit via la propriété de cristal un isomorphisme

$$
E_{K} \simeq M \otimes_{\vartheta_{K}} A_{\text {cris }, K}
$$

via lequel

$$
\text { Fil } E_{K}=\text { Fil } M \otimes_{\vartheta_{K}} A_{\text {cris }, K}+M \otimes_{\vartheta_{K}} \text { Fil }^{1} A_{\text {cris }, K} .
$$

Observons maintenant que cette description de la filtration induit un scindage canonique de l'analogue de la filtration apparaissant dans la démonstration du théorème 2 :

$$
\mathrm{Fil}^{0} E_{K} / \mathrm{Fil}^{1} E_{K} \stackrel{\sim}{\longrightarrow} M / \text { Fil } M \otimes_{\vartheta_{K}} \operatorname{gr}^{1} A_{\text {cris }, K} \oplus \text { Fil } M \otimes_{\vartheta_{K}} \operatorname{gr}^{0} A_{\text {cris }, K} .
$$

L'application des périodes $T_{p}(H) \otimes A_{\text {cris }, K} \rightarrow$ Fil $E_{K}$ induit un morphisme $\operatorname{Gal}(\bar{K} \mid K)$-équivariant de $\theta_{\widehat{K}}$-modules

$$
\begin{aligned}
T_{p}(H) \otimes_{\mathbb{Z}_{p}} \vartheta_{\widehat{\bar{K}}} \longrightarrow \mathrm{Fil}^{0} E_{K} / \mathrm{Fil}^{1} E_{K} & =\left(\omega_{H}^{*} \otimes \operatorname{gr}^{1} A_{\text {cris }, K}\right) \oplus\left(\omega_{H^{D}} \otimes \operatorname{gr}^{0} A_{\text {cris }, K}\right) \\
& =\omega_{H}^{*} \otimes T_{p}\left(\Omega_{\vartheta_{\bar{K}} / \oslash_{K}}\right) \oplus \omega_{H^{D}} \otimes \vartheta_{\widehat{\bar{K}}}
\end{aligned}
$$

On vérifie comme dans la preuve du théorème 2 que ce morphisme est injectif de conoyau annulé par tout élément de valuation $\frac{1}{p-1}+v\left(\mathscr{D}_{K / K_{0}}\right)$. De plus, la composée

$$
\omega_{H}^{*} \otimes \theta_{\widehat{K}}(1) \stackrel{{ }^{t}\left(\alpha_{H} D \otimes \operatorname{Id}\right)(1)}{\longrightarrow} T_{p}(H) \otimes \vartheta_{\widehat{K}} \longrightarrow \omega_{H}^{*} \otimes T_{p}\left(\Omega_{\vartheta_{\bar{K}} / \vartheta_{K}}\right)
$$

est donnée par l'inclusion $\emptyset_{\widehat{K}}(1) \hookrightarrow \operatorname{gr}^{1} A_{\text {cris }, K}$. On retrouve donc le résultat de [18] sur le presque-scindage de la suite de Hodge-Tate d'un groupe $p$-divisible.

\section{La filtration des points de $p$-torsion : une borne effective}

\subsection{Notations}

Dans ce chapitre on suppose $K$ algébriquement clos. Puisque les filtrations que nous étudions descendent automatiquement de $\widehat{\bar{K}}$ à $K$, on peut toujours se ramener à ce cas-là pour les problèmes qui nous intéressent. 


\subsection{Un lemme d'approximation d'Elkik}

Lemme 7. - Soit $(A, \Im)$ un couple hensélien tel que A soit séparé pour la topologie I-adique et l'idéal I de type fini engendré par une suite régulière. Notons $S=\operatorname{Spec}(A)$. Soit $X$ un $S$-schéma fini et plat étale sur un ouvert schématiquement dense et globalement intersection complète dans un espace affine $\mathbb{A}_{S}^{N}$. Notons

$$
D=\operatorname{Div}\left(\mathbb{L}_{X / S}\right),
$$

un diviseur de Cartier sur $X$. Soient $n$ et $h$ deux entiers tels que $n>2 h$. L'application de réduction modulo $\mathfrak{I}^{n-h}$ induit alors une bijection

$$
\left.\left\{x \in X(A) \mid \mathfrak{I}^{h} \subset x^{*} D\right\} \stackrel{\sim}{\longrightarrow} \operatorname{Im}\left[\left\{x \in X\left(A / \mathfrak{I}^{n}\right) \mid \mathfrak{I}^{h} \subset x^{*} D\right\} \rightarrow X\left(A / \mathfrak{I}^{n-h}\right\}\right)\right] .
$$

Démonstration. - L'assertion de surjectivité résulte du lemme 2 de [12]. Montrons l'injectivité. Soit

$$
X=\operatorname{Spec}\left(A\left[X_{1}, \ldots, X_{N}\right] /\left(f_{1}, \ldots, f_{N}\right)\right)
$$

Notons

$$
M=\left(\frac{\partial f_{i}}{\partial X_{j}}\right)_{1 \leq i, j \leq N}
$$

la matrice jacobienne et $\Delta=\operatorname{det}(M)$ l'idéal jacobien. En restriction à $X$, le polynôme $\Delta$ engendre le diviseur $D$. Soient $\underline{x}, \underline{y} \in A^{N}$ tels que pour tout entier $i$ tel que $1 \leq i \leq N$ on ait les égalités

ainsi que les inclusions

$$
f_{i}(\underline{x})=0, f_{i}(\underline{y})=0
$$

$$
\mathfrak{I}^{h} \subset \Delta(\underline{x}) \text { et } \mathfrak{I}^{h} \subset \Delta(\underline{y})
$$

Supposons que

$$
\underline{x} \equiv \underline{y}\left[\mathfrak{I}^{n-h}\right] .
$$

Montrons par récurrence sur $k \geq 0$ que

$$
\underline{x} \equiv \underline{y}\left[\mathfrak{I}^{2^{k}(n-2 h)+h}\right] .
$$

Cela conclura la preuve puisque $A$ est séparé pour la topologie I-adique. Supposons l'hypothèse vérifiée au rang $k$. Écrivant un développement de Taylor à l'ordre 1, on obtient la relation de congruence

$$
\left(\begin{array}{c}
f_{1}(\underline{y}) \\
\vdots \\
f_{N}(\underline{y})
\end{array}\right) \equiv\left(\begin{array}{c}
f_{1}(\underline{x}) \\
\vdots \\
f_{N}(\underline{x})
\end{array}\right)+M(\underline{x})\left(\begin{array}{c}
y_{1}-x_{1} \\
\vdots \\
y_{N}-x_{N}
\end{array}\right) \bmod \mathfrak{I}^{\mathfrak{I}^{k+1}(n-2 h)+2 h}
$$

et donc

$$
M(\underline{x})\left(\begin{array}{c}
y_{1}-x_{1} \\
\vdots \\
y_{N}-x_{N}
\end{array}\right) \equiv 0 \bmod \mathfrak{I}^{2^{k+1}(n-2 h)+2 h} .
$$

En multipliant par la matrice des cofacteurs de $M(\underline{x})$ on obtient, pour tout entier $i$ tel que $1 \leq i \leq N$, la relation de congruence

$$
\Delta(\underline{x}) \cdot\left(y_{i}-x_{i}\right) \equiv 0 \bmod \mathfrak{I}^{2^{k+1}(n-2 h)+2 h}
$$

$4^{\text {e }}$ SÉRIE - TOME $44-2011-$ No $^{\circ}$ 
et donc

$$
\left(y_{i}-x_{i}\right) \mathfrak{I}^{h} \subset \mathfrak{I}^{2^{k+1}(n-2 h)+2 h} .
$$

Le choix d'une suite régulière engendrant $\mathscr{I}$ définit un isomorphisme (EGA IV, 15.1.9)

$$
A / \mathfrak{I}\left[T_{1}, \ldots, T_{d}\right] \stackrel{\sim}{\longrightarrow} \mathrm{Gr}_{\mathfrak{I}}^{\bullet} A .
$$

De cela on déduit qu'on peut «simplifier » la relation de congruence précédente par $\mathfrak{I}^{h}$ pour obtenir

$$
x_{i} \equiv y_{i} \bmod \mathfrak{I}^{2^{k+1}(n-2 h)+h}
$$

ce qui est le résultat escompté.

\subsection{Préliminaires d'algèbre linéaire}

Voici l'analogue des propositions 5.1 de [1] et 9.1 de [3].

Proposition 7. - Soit $M$ un $\theta_{K, 1}$-module libre de rang $n$ muni d'un morphisme $\psi: M \longrightarrow M^{(p)}$ vérifiant

Soit

$$
w:=\operatorname{deg}(\operatorname{coker} \psi)<\frac{1}{2}
$$

$$
N=\{m \in M \mid \psi(m)=m \otimes 1\},
$$

un sous $-\mathbb{F}_{p}$-espace vectoriel de $M$. Alors,

1. Le sous $-\mathbb{F}_{p}$-espace vectoriel de $M_{1-w}$

$$
V:=\operatorname{Im}\left(N \longrightarrow M_{1-w}\right)
$$

est de dimension n. De plus pour tout $\epsilon \in v\left(K^{\times}\right)$vérifiant $\frac{w}{p-1}<\epsilon \leq 1-w$, V s'injecte dans $M_{\epsilon}$.

2. Si $v_{1}, \ldots, v_{n}$ est une base de $V,\left(\lambda_{1}, \ldots, \lambda_{n}\right) \in \theta_{K}^{n}$, $\epsilon$ vérifie $\frac{w}{p-1}<\epsilon \leq 1-w$ et il y a une relation

$$
\sum_{i=1} \lambda_{i} v_{i}=0 \text { dans } M_{\epsilon}
$$

alors pour tout $i \in\{1, \ldots, n\}, v\left(\lambda_{i}\right)>0$.

3. Soit $f: V \otimes_{\mathbb{F}_{p}} \theta_{K, 1-w} \longrightarrow M_{1-w}$ l'application naturelle. Il existe alors un morphisme de $\vartheta_{K, 1-w}$-modules $g: M_{1-w} \longrightarrow V \otimes_{\mathbb{F}_{p}} \theta_{K, 1-w}$ tel que $f \circ g=\delta \operatorname{Id}$ et $g \circ f=\delta \operatorname{Id}$ où $\delta \in \vartheta_{K}$ vérifie $v(\delta)=\frac{w}{p-1}$.

4. Pour tout $\epsilon \in v\left(K^{\times}\right)$vérifiant $\frac{w}{p-1}<\epsilon \leq 1-w, \operatorname{deg}\left(M_{\epsilon} / \theta_{K} \cdot \operatorname{Im}\left(V \rightarrow M_{\epsilon}\right)\right)=\frac{w}{p-1}$.

Démonstration. - Fixons une base de $M$. Pour $X=\left(x_{i}\right)_{1 \leq i \leq n} \in \theta_{K}^{n}$, notons $X^{(p)}=\left(x_{i}^{p}\right)_{1 \leq i \leq n}$. Soit $A \in M_{n}\left(\theta_{K}\right)$ une matrice se réduisant modulo $p$ sur la matrice de $\psi$. L'ensemble $N$ s'identifie aux solutions de la congruence

$$
X^{(p)} \equiv A X \bmod p
$$

avec $X \in \theta_{K, 1}^{n}$. Le lemme 7 s'applique et montre que l'application de réduction modulo $\mathfrak{m}_{K, 1-w}$ induit une bijection

$$
\left\{X \in \theta_{K}^{n} \mid X^{(p)}=A X\right\} \stackrel{\sim}{\longrightarrow} V .
$$


Plus précisément, si $A=\left(a_{i j}\right)_{1 \leq i, j \leq n}$, on applique le lemme 7 à la variété algébrique affine d'anneau

$$
\oslash_{K}\left[X_{1}, \ldots, X_{n}\right] /\left(X_{i}^{p}-\sum_{j=1}^{n} a_{i j} X_{j}\right)_{1 \leq i \leq n}
$$

(à strictement parler le lemme 7 s'applique lorsque $w \in \mathbb{Q}$ mais le lecteur n'aura aucune difficulté à en déduire le résultat pour $w$ quelconque en l'écrivant comme limite de nombres rationnels).

L'ensemble de gauche dans la bijection (5) s'identifie aux $\theta_{K}$-points d'une $\theta_{K}$-algèbre finie et libre de rang $p^{n}$ étale en dehors de $p$. Il est donc de cardinal $p^{n}$ ce qui implique que $\operatorname{dim}_{\mathbb{F}_{p}} V=n$.

Montrons maintenant que, pour $\epsilon$ comme dans l'énoncé, $V$ s'injecte dans $M_{\epsilon}$. Soit $X \in \theta_{K}^{n}$ non nul tel que $X^{(p)}=A X$. Écrivons $X=\left(x_{1}, \ldots, x_{n}\right)$ et soit $\alpha=\inf \left\{v\left(x_{i}\right) \mid 1 \leq i \leq n\right\}$. Soit $B$ la matrice des cofacteurs de $A$. L'équation précédente fournit l'équation

$$
B X^{(p)}=\operatorname{det}(A) X .
$$

Celle-ci implique l'inégalité

$$
p \alpha \leq w+\alpha
$$

et donc $\alpha \leq \frac{w}{p-1}$. Cela implique que $V$ s'injecte dans $M_{\epsilon}$ dès que $\frac{w}{p-1}<\epsilon \leq 1-w$.

Montrons maintenant le point (2) de l'énoncé. Soient $x_{1}, \ldots, x_{n} \in N$ induisant une base de $V$. Soit $f: M^{(p)} \longrightarrow M$ un morphisme $\theta_{K}$-linéaire tel que $f \circ \psi=a \operatorname{Id}$ où $v(a)=w$. Définissons un morphisme Frob-linéaire $\varphi: M \rightarrow M$ en posant $\varphi(x)=f(x \otimes 1)$. Pour $1 \leq i \leq n$ on a alors $\varphi\left(x_{i}\right)=a x_{i}$. Si $\lambda_{1}, \ldots, \lambda_{n} \in \theta_{K}$ sont tels que $\sum_{i=1}^{n} \lambda_{i} x_{i}=0$ dans $M_{\epsilon}$, par application de $\varphi$ on obtient que $a \sum_{i=1}^{n} \lambda_{i}^{p} x_{i}=0$ dans $M_{\inf \{1, p \epsilon\}}$ et donc $\sum_{i=1}^{n} \lambda_{i}^{p} x_{i}=0$ dans $M_{\inf \{1, p \epsilon\}-w}$. Grâce à l'hypothèse faite sur $\epsilon$, on a $\inf \{1, p \epsilon\}-w \geq \epsilon$. On obtient donc que $\sum_{i=1}^{n} \lambda_{i}^{p} x_{i}=0$ dans $M_{\epsilon}$. À partir de là la suite de la preuve du point (2) est strictement identique à celle du lemme 9.7 de [3] auquel on renvoie.

Montrons maintenant le point (3) de la proposition. Soit $C \in M_{n}\left(\theta_{K}\right)$ une matrice dont les colonnes vérifient l'équation $X^{(p)}=A X$ et induisent par réduction une base de $V$. Le point (2) implique que $\operatorname{det}(C) \neq 0$. De plus, l'équation

$$
C^{(p)}=A C
$$

implique l'égalité $v(\operatorname{det} C)=\frac{w}{p-1}$. Les points (3) et et (4) s'en déduisent.

Nous utiliserons le lemme suivant. On renvoie au lemme 14 de [16] pour la preuve de celui-ci.

Lemme 8. - Soient $M$ un $\theta_{K}$-module de présentation finie et $N \subset M$ un sous-module de type fini. Soit $r \geq 1$ un entier. Si $M$ est engendré par $r$ éléments, alors $N$ est engendré par $r$ éléments.

La proposition qui suit sera importante dans la preuve du théorème 4.

4 e SÉRIE - TOME $44-2011-$ No $^{\circ}$ 
Proposition 8. - Soit $M$ un $\theta_{K}$-module de présentation finie de torsion muni d'une filtration croissante

$$
0=\mathrm{Fil}_{0} M \subsetneq \mathrm{Fil}_{1} M \subsetneq \cdots \subsetneq \mathrm{Fil}_{r-1} M \subsetneq \mathrm{Fil}_{r} M=M
$$

dont les gradués sont monogènes. Supposons donnée une collection d'éléments $\left(x_{i}\right)_{1 \leq i \leq r}$ d'éléments de $M$ telle que, pour tout $i, x_{i} \in \operatorname{Fil}_{i} M$ et vérifiant pour $\left(\lambda_{i}\right)_{1 \leq i \leq r} \in \theta_{K}^{r}$,

$$
\sum_{i=1}^{r} \lambda_{i} x_{i}=0 \Rightarrow \forall i, v\left(\lambda_{i}\right)>0 .
$$

Alors, pour $1 \leq i \leq r$, l'image $\bar{x}_{i}$ de $x_{i}$ dans $\mathrm{Fil}_{i} M / \mathrm{Fil}_{i-1} M$ est non-nulle et

$$
\operatorname{deg}\left(M / \sum_{i=1}^{r} \vartheta_{K} \cdot x_{i}\right)=\sum_{i=1}^{r} \operatorname{deg}\left(\left(\operatorname{Fil}_{i} M / \operatorname{Fil}_{i-1} M\right) / \vartheta_{K} \cdot \bar{x}_{i}\right) .
$$

Démonstration. - Il suffit de montrer la propriété

$$
\left(*_{r}\right) \quad \forall 1 \leq j \leq r, \quad \operatorname{Fil}_{j} M \cap \sum_{i=1}^{r} \vartheta_{K} \cdot x_{i}=\sum_{i=1}^{j} \vartheta_{K} \cdot x_{i} .
$$

En effet, si la propriété $\left(*_{r}\right)$ est vérifiée et que l'on avait un indice $i$ tel que $x_{i} \in \mathrm{Fil}_{i-1} M$, i.e. $\bar{x}_{i}=0$, il existerait $\lambda_{1}, \ldots, \lambda_{i-1} \in \theta_{K}$ tels que

$$
x_{i}=\sum_{k=1}^{i-1} \lambda_{k} x_{k}
$$

et donc $x_{i}-\sum_{k=1}^{i-1} \lambda_{k} x_{k}=0$, ce qui est impossible par hypothèse. De plus, la propriété $\left(*_{r}\right)$ implique que l'on a une suite exacte

$$
0 \longrightarrow \mathrm{Fil}_{r-1} M / \sum_{i=1}^{r-1} \theta_{K} \cdot x_{i} \longrightarrow M / \sum_{i=1}^{r} \theta_{K} \cdot x_{i} \longrightarrow\left(\mathrm{Fil}_{r} M / \mathrm{Fil}_{r-1} M\right) / \vartheta_{K} \cdot \bar{x}_{r} \longrightarrow 0 .
$$

Par additivité de la fonction degré et par récurrence on en déduit l'assertion de la proposition concernant l'égalité des degrés.

Montrons maintenant la propriété $\left(*_{r}\right)$ par récurrence sur $r$. Supposons-la vérifiée au rang $r-1$. Soient $\lambda_{1}, \ldots, \lambda_{r} \in \theta_{K}$ tels que

$$
y=\sum_{i=1}^{r} \lambda_{i} x_{i} \in \operatorname{Fil}_{j} M
$$

avec $1 \leq j<r$. On a donc

$$
\lambda_{r} x_{r} \in \mathrm{Fil}_{r-1} M .
$$

Puisque $\mathrm{Fil}_{r-1} M / \mathrm{Fil}_{r-2} M$ est monogène, il existe $\alpha, \beta \in \theta_{K}$ tels que

$$
\alpha \lambda_{r} x_{r}+\beta x_{r-1} \in \mathrm{Fil}_{r-2} M
$$

avec soit $\alpha=1$, soit $\beta=1$. Supposons d'abord que $\beta=1$ i.e. $\alpha \lambda_{r} x_{r}+x_{r-1} \in \mathrm{Fil}_{r-2} M$. On vérifie aisément que le module $\mathrm{Fil}_{r-1} M$ muni de la filtration $\left(\mathrm{Fil}_{i} M\right)_{0 \leq i \leq r-1}$ et des éléments $\left(x_{1}, \ldots, x_{r-2}, \alpha \lambda_{r} x_{r}+x_{r-1}\right)$ satisfait à l'hypothèse de récurrence. D'après le raisonnement précédent (cf. début de la démonstration) cela implique que $\alpha \lambda_{r} x_{r}+x_{r-1} \notin \mathrm{Fil}_{r-2} M$, ce qui est une contradiction. On a donc $\alpha=1$ et $\lambda_{r} x_{r}+\beta x_{r-1} \in \mathrm{Fil}_{r-2} M$. 
Montrons maintenant par récurrence descendante sur $k$ à partir de $k=r-1$ qu'il existe $\beta_{k}, \ldots, \beta_{r-1} \in \theta_{K}$ tels que

$$
\lambda_{r} x_{r}+\beta_{k} x_{k}+\cdots+\beta_{r-1} x_{r-1} \in \mathrm{Fil}_{k-1} M .
$$

Le cas initial $k=r-1$ vient d'être vérifié. Supposons donc vérifiée l'hypothèse de récurrence au rang $k$. Notons

$$
z=\lambda_{r} x_{r}+\beta_{k} x_{k}+\cdots+\beta_{r-1} x_{r-1} \in \mathrm{Fil}_{k-1} M .
$$

Puisque le module $\mathrm{Fil}_{k-1} M / \mathrm{Fil}_{k-2} M$ est monogène il existe $\alpha, \gamma \in \theta_{K}$ tels que

$$
\gamma x_{k-1}+\alpha z \in \mathrm{Fil}_{k-2} M
$$

avec $\alpha=1$ ou bien $\gamma=1$. Si l'on avait $\gamma=1$, on pourrait appliquer l'hypothèse de récurrence sur $r$ au module filtré $\mathrm{Fil}_{k-1} M$ muni des éléments $\left(x_{1}, \ldots, x_{k-2}, x_{k-1}+\alpha z\right)$. Comme précédemment cela est impossible. On a donc $\alpha=1$ et l'hypothèse de récurrence au rang $k-1$ est vérifiée.

Appliquant le résultat que l'on vient d'établir à $k=1$ on obtient que

$$
\lambda_{r} x_{r} \in \sum_{i=1}^{r-1} \vartheta_{K} \cdot x_{i} .
$$

On a donc

$$
y \in \sum_{i=1}^{r-1} \vartheta_{K} x_{i} \cap \operatorname{Fil}_{j} M .
$$

De cela on déduit par application de l'hypothèse de récurrence $\left(*_{r-1}\right)$ que $y \in \sum_{i=1}^{j} \vartheta_{K} x_{i}$.

\subsection{Sur l'application de Hodge-Tate des points de $p$-torsion d'un groupe $p$-divisible}

Nous allons maintenant utiliser le théorème-clef 3.

Définition 6. - Pour $\epsilon \in v\left(K^{\times}\right), 0<\epsilon \leq 1$, on note $\alpha_{G, \epsilon}$ l'application composée

$$
G\left(\theta_{K}\right) \stackrel{\alpha_{G}}{\longrightarrow} \omega_{G^{D}} \longrightarrow \omega_{G^{D}, \epsilon} .
$$

Proposition 9. - Soit G un groupe de Barsotti-Tate tronqué d'échelon 1 et de dimension d sur $\theta_{K}$. Pour $\frac{1}{p-1}<\epsilon \leq 1$, l'inégalité suivante est vérifiée :

$$
\operatorname{dim}_{\mathbb{F}_{p}} \operatorname{ker} \alpha_{G, \epsilon} \leq d .
$$

Démonstration. - Soit $h$ la hauteur de $G$. Puisque $G$ est un groupe de Barsotti-Tate tronqué d'échelon $1, \omega_{G^{D}} \simeq\left(\vartheta_{K} / p \vartheta_{K}\right)^{h-d}$. On en déduit que si $\delta \in \vartheta_{K}$ est un élément de valuation $\frac{1}{p-1}, \delta \omega_{G^{D}, \epsilon}$ est un $\Theta_{K, \epsilon-\frac{1}{p-1}}$-module libre de rang $h-d$. D'après le théorème 3 ,

$$
\delta \omega_{G^{D}, \epsilon} \subset \theta_{K} \cdot \operatorname{Im}\left(\alpha_{G, \epsilon}\right) .
$$

Puisque $\theta_{K} \cdot \operatorname{Im}\left(\alpha_{G, \epsilon}\right)$ est un sous-module de type fini du module de présentation finie $\omega_{G^{D}, \epsilon}$, c'est un module de présentation finie. On peut alors appliquer le lemme 8 pour conclure que $\delta \omega_{G^{D}, \epsilon}$ est engendré par moins de $\operatorname{dim}_{\mathbb{F}_{p}} \operatorname{Im}\left(\alpha_{G}\right)$ éléments et que donc

$$
h-d \leq \operatorname{dim}_{\mathbb{F}_{p}} \operatorname{Im}\left(\alpha_{G}\right) .
$$

$4^{\text {e }}$ SÉRIE - TOME $44-2011$ - No 6 
On renvoie à la proposition 13 de la section 7.2 pour un énoncé plus général concernant les groupes de Barsotti-Tate tronqués d'échelon quelconque.

\subsection{Sous-groupe canonique des groupes de Barsotti-Tate tronqués d'échelon 1}

Proposition 10. - Supposons $p \neq 2$. Soit $G$ un groupe de Barsotti-Tate tronqué d'échelon 1 , de hauteur $h$ et de dimension $d<h$ sur $\Theta_{K}$. Supposons vérifiée l'inégalité

$$
\mathrm{Ha}(G)<\frac{1}{2} \text {. }
$$

Alors, si

on a l'égalité

$$
\frac{\mathrm{Ha}(G)}{p-1}<\epsilon \leq 1-\mathrm{Ha}(G)
$$

$$
\operatorname{dim}_{\mathbb{F}_{p}} \operatorname{ker}\left(\alpha_{G, \epsilon}\right)=d .
$$

Démonstration. - Posons $w=\mathrm{Ha}(G)$. D'après la proposition 9, puisque $1-w>\frac{1}{p-1}$, on a l'inégalité $\operatorname{dim}_{\mathbb{F}_{p}} \operatorname{Im}\left(\alpha_{G, 1-w}\right) \geq h-d$. D'après le lemme 3 et la proposition 7, l'image de $\alpha_{G, 1-w}$ est contenue dans un $\mathbb{F}_{p}$-espace vectoriel $V$ de dimension $h-d$. On a donc $\operatorname{dim}_{\mathbb{F}_{p}} \operatorname{Im}\left(\alpha_{G, 1-w}\right)=h-d$ et $V=\operatorname{Im}\left(\alpha_{G, 1-w}\right)$. Le point (1) de la proposition 7 dit que $V$ s'injecte dans $\omega_{G^{D}, \epsilon}$ pour tout $\epsilon$ comme dans l'énoncé. Cela permet de conclure.

Remarque 3. - Dans la proposition 10 la preuve de l'inégalité $\operatorname{dim}_{\mathbb{F}_{p}} \operatorname{ker} \alpha_{G, \epsilon} \leq d$ utilise de façon essentielle le fait que G est un groupe de Barsotti-Tate tronqué et que le conoyau de l'application de Hodge-Tate est annulé par $p^{\frac{1}{p-1}}$. C'est là l'ingrédient nouveau que nous apportons à [1], [36] et [3]. Comme l'a fait remarquer l'un des rapporteurs à l'auteur, l'inégalité dans l'autre sens, $\operatorname{dim}_{\mathbb{F}_{p}} \operatorname{ker} \alpha_{G, 1-\mathrm{Ha}(G)} \geq d$ résulte en fait de [36] et [3] et n'est pas liée au fait que $G$ soit un $B T_{1}$. Cette partie n'est donc pas nouvelle. Plus précisément, dans le cas des points de p-torsion d'un schéma abélien, c'est une conséquence du théorème 8.1 et de la proposition 9.1 de [3]. Le cas d'un groupe fini et plat annulé par p général peut alors se déduire du cas précédent par un dévissage standard en utilisant la proposition 5.5(i) de [36].

Lemme 9. - Soit $G$ un groupe de hauteur 1 sur $\theta_{K}$. Soit $x \in G\left(\theta_{K}\right)$ un générateur $d u$ groupe cyclique $G\left(\theta_{K}\right)$. Il existe alors un isomorphisme $\omega_{G^{D}} \simeq \theta_{K} / \gamma \vartheta_{K}$ avec $\gamma \in \theta_{K}$, ainsi qu'un élément $y \in \theta_{K}$, tels que via l'isomorphisme précédent, $\alpha_{G}(x)=y \bmod \gamma \theta_{K}$ et

$$
\begin{aligned}
& v(\gamma)=1-\operatorname{deg}(G) \\
& v(y)=\frac{\operatorname{deg} G}{p-1} .
\end{aligned}
$$

Démonstration. - Le schéma en groupes $G$ est du type de ceux étudiés par Oort et Tate dans [35]. On peut calculer explicitement l'application de Hodge-Tate d'un tel schéma en groupes (cf. [14] chapitre 1.1.1).

ThÉORÈme 4. - Supposons $p \neq 2$. Soit $G$ un groupe de Barsotti-Tate tronqué d'échelon 1 , de hauteur $h$ et de dimension $d<h$ sur $\vartheta_{K}$. Supposons vérifiée l'inégalité

$$
\mathrm{Ha}(G)<\frac{1}{2}
$$


L'égalité suivante est alors vérifiée

$$
\operatorname{dim}_{\mathbb{F}_{p}} \operatorname{ker} \alpha_{G, 1-\mathrm{Ha}(G)}=d .
$$

Si $p=3$ supposons de plus que $\mathrm{Ha}(G)<\frac{1}{3}$. On a alors les propriétés suivantes.

1. Soit $C$ l'adhérence schématique de $\operatorname{ker} \alpha_{G, 1-\mathrm{Ha}(G)}$ dans $G$. L'égalité suivante est alors vérifiée

$$
\operatorname{deg}\left(C^{D}\right)=\operatorname{deg}(G / C)=\operatorname{Ha}(G) .
$$

2. Soit $\left(G_{\mathrm{rn}}^{\lambda}\right)_{\lambda>0}$ la filtration de ramification naïve de $G$ (cf. définition 3$)$ et $\left(\left(G^{D}\right)_{\mathrm{AS}}^{\lambda}\right)_{\lambda>0}$ celle d'Abbes-Saito de $G^{D}$ (cf. définition 4). Posons $w:=\mathrm{Ha}(G)$. Alors, pour $\lambda$ vérifiant $\frac{w}{p-1} \leq \lambda<\frac{1-w}{p-1}$, on a l'égalité

$$
G_{\mathrm{rn}}^{\lambda}=C .
$$

Pour $\lambda$ vérifiant $\frac{p w}{p-1} \leq \lambda<\frac{p}{p-1}(1-w)$, on a l'égalité

$$
\left(G^{D}\right)_{\mathrm{AS}}^{\lambda}\left(\theta_{K}\right)=C\left(\theta_{K}\right)^{\perp}
$$

3. Le sous-groupe plat fini $C \otimes \ominus_{K, 1-w} \subset G \otimes \vartheta_{K, 1-w}$ coïncide avec le noyau du morphisme de Frobenius de $G \otimes \theta_{K, 1-w}$.

Démonstration. - La première assertion est contenue dans la proposition 10.

Montrons le point (1) de l'énoncé. Posons $E=G / C$. Il y a un morphisme de suites exactes

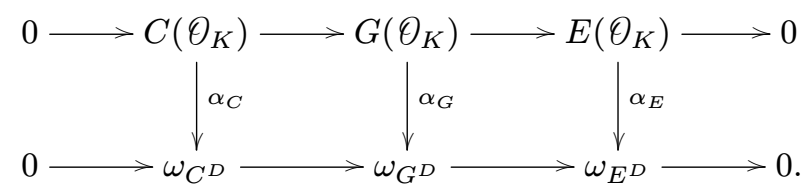

On en déduit en particulier que $\mathfrak{m}_{K, w} \cdot \operatorname{Im}\left(\alpha_{C} \otimes 1\right)=0$. Le théorème 3 appliqué à $C$ implique que $\mathfrak{m}_{K, \frac{1}{p-1}} \cdot \omega_{C^{D}} \subset \operatorname{Im}\left(\alpha_{C} \otimes 1\right)$. On en déduit donc que

$$
\mathfrak{m}_{K, w+\frac{1}{p-1}} \cdot \omega_{C^{D}}=0 .
$$

Soit $\epsilon \in v\left(K^{\times}\right)$tel que $\frac{w}{p-1}<\epsilon \leq 1-w$ et $\epsilon \leq 1-\frac{1}{p-1}-w$ (il existe toujours un tel $\epsilon$ grâce à l'hypothèse faite lorsque $p=3$ ). La projection $\omega_{G^{D}} \longrightarrow \omega_{E^{D}}$ induit un isomorphisme

$$
\omega_{G^{D}, \epsilon} \stackrel{\sim}{\longrightarrow} \omega_{E^{D}, \epsilon} .
$$

Soit $\left(e_{1}, \ldots, e_{h-d}\right)$ une base du $\mathbb{F}_{p}$-espace vectoriel $E\left(\theta_{K}\right)$. Notons, pour $1 \leq i \leq h-d$, $E_{i}$ l'adhérence schématique de $\mathbb{F}_{p} e_{1}, \oplus \cdots \oplus \mathbb{F}_{p} e_{i}$ dans $E$. Il y a donc un drapeau de groupes plats finis

$$
(0)=E_{0} \subsetneq E_{1} \subsetneq \cdots \subsetneq E_{h-d}=E,
$$

dont les gradués sont des groupes de hauteur 1. Celui-ci induit un drapeau de $\theta_{K}$-modules

$$
(0)=\omega_{E_{0}^{D}} \subset \omega_{E_{1}^{D}} \subset \cdots \subset \omega_{E_{h-d}^{D}}=\omega_{E^{D}}
$$

dont les gradués sont des $\theta_{K}$-modules monogènes. Notons $\left(\operatorname{Fil}_{i} \omega_{E^{D}, \epsilon}\right)_{0 \leq i \leq h-d}$ la filtration image

$$
\operatorname{Fil}_{i} \omega_{E^{D}, \epsilon}=\operatorname{Im}\left(\omega_{E_{i}^{D}, \epsilon} \longrightarrow \omega_{E^{D}, \epsilon}\right) .
$$

Pour $1 \leq i \leq h-d$ il y a une surjection canonique

$$
q_{i}: \omega_{\left(E_{i} / E_{i-1}\right)^{D}, \epsilon} \rightarrow \operatorname{Fil}_{i} \omega_{E^{D}, \epsilon} / \operatorname{Fil}_{i-1} \omega_{E^{D}, \epsilon} .
$$


D'après le point (2) de la proposition 7, la collection d'éléments $\left(\alpha_{E, \epsilon}\left(e_{i}\right)\right)_{1 \leq i \leq h-d}$ satisfait aux hypothèses de la proposition 8 . On en déduit que pour $1 \leq i \leq h-d$,

$$
q_{i} \circ \alpha_{E_{i} / E_{i-1}, \epsilon}\left(\bar{e}_{i}\right) \neq 0 \in \operatorname{Fil}_{i} \omega_{E^{D}, \epsilon} / \operatorname{Fil}_{i-1} \omega_{E^{D}, \epsilon}
$$

où $\bar{e}_{i} \in\left(E_{i} / E_{i-1}\right)\left(\theta_{K}\right)$ désigne l'image de $e_{i} \in E_{i}\left(\theta_{K}\right)$. On a donc en particulier

$$
\alpha_{E_{i} / E_{i-1}, \epsilon}\left(\bar{e}_{i}\right) \neq 0 \in \omega_{\left(E_{i} / E_{i-1}\right)^{D}, \epsilon} .
$$

D’après le lemme 9, cela implique que

$$
\operatorname{deg}\left(\omega_{\left(E_{i} / E_{i-1}\right)^{D}, \epsilon} / \theta_{K} \cdot \alpha_{E_{i} / E_{i-1}, \epsilon}\left(e_{i}\right)\right)=\frac{\operatorname{deg}\left(E_{i} / E_{i-1}\right)}{p-1} .
$$

Mais puisque l'application $q_{i}$ est un morphisme surjectif de $\theta_{K}$-modules monogènes et $q_{i} \circ \alpha_{E_{i} / E_{i-1}, \epsilon}\left(\bar{e}_{i}\right) \neq 0$ on a $\operatorname{deg}\left(\omega_{\left(E_{i} / E_{i-1}\right)^{D}, \epsilon} / \vartheta_{K} \cdot \alpha_{E_{i} / E_{i-1}, \epsilon}\left(e_{i}\right)\right)=\operatorname{deg}\left(\left(\operatorname{Fil}_{i} \omega_{E^{D}, \epsilon} / \operatorname{Fil}_{i-1} \omega_{E^{D}, \epsilon}\right) / \vartheta_{K} \cdot q_{i} \circ \alpha_{E_{i} / E_{i-1}, \epsilon}\left(\bar{e}_{i}\right)\right)$.

Le dernier point de la proposition 8 fournit alors l'égalité

$$
\begin{aligned}
\operatorname{deg} \operatorname{coker}\left(\alpha_{E, \epsilon} \otimes 1\right) & =\sum_{i=1}^{h-d} \operatorname{deg}\left(\left(\operatorname{Fil}_{i} \omega_{E^{D}, \epsilon} / \operatorname{Fil}_{i-1} \omega_{E^{D}, \epsilon}\right) / \theta_{K} \cdot q_{i} \circ \alpha_{E_{i} / E_{i-1}, \epsilon}\left(\bar{e}_{i}\right)\right) \\
& =\sum_{i=1}^{h-d} \frac{\operatorname{deg}\left(E_{i} / E_{i-1}\right)}{p-1} \\
& =\frac{\operatorname{deg} E}{p-1} .
\end{aligned}
$$

Mais d'après le point (4) de la proposition 7 on a

$$
\operatorname{deg} \operatorname{coker}\left(\alpha_{E, \epsilon} \otimes 1\right)=\frac{w}{p-1} .
$$

On obtient donc bien que $\operatorname{deg} E=w$.

Montrons le point (2) de l'énoncé. Pour $x \in C\left(\theta_{K}\right) \backslash\{0\}$ notons $\langle x\rangle$ l'adhérence schématique du sous-groupe de $C\left(\theta_{K}\right)$ engendré par $x$ dans $C$. Soit donc $x \in C\left(\theta_{K}\right) \backslash\{0\}$. D'après le point (1), $\mathfrak{m}_{K, w} \cdot \omega_{C^{D}}=0$. Puisque $\omega_{\langle x\rangle^{D}} \hookrightarrow \omega_{C^{D}}$ on a donc $\mathfrak{m}_{K, w} \cdot \omega_{\langle x\rangle^{D}}=0$. On en déduit que

$$
\operatorname{deg}(\langle x\rangle) \geq 1-w
$$

Maintenant si $x \in G\left(\theta_{K}\right) \backslash C\left(\theta_{K}\right)$ et $y \in E\left(\theta_{K}\right)$ désigne la projection de $x$, le morphisme naturel de schémas en groupes $\langle x\rangle \rightarrow\langle y\rangle$ est un isomorphisme en fibre générique. On a donc $\operatorname{deg}(\langle x\rangle) \leq \operatorname{deg}(\langle y\rangle)$. Pour $\epsilon>\frac{w}{p-1}$ suffisamment proche de $\frac{w}{p-1}, \alpha_{E, \epsilon}$ et donc les $\alpha_{\langle y\rangle, \epsilon}$ sont injectifs. On déduit alors du lemme 9 que $\operatorname{deg}(\langle y\rangle) \leq w$. On a donc, pour $x \in G\left(\theta_{K}\right) \backslash C\left(\theta_{K}\right)$,

$$
\operatorname{deg}(\langle x\rangle) \leq w
$$

Les propositions 5 et 6 permettent de conclure.

Montrons maintenant le point (3) de l'énoncé. Puisque d'après le point (1) $\operatorname{deg}\left(C^{D}\right)=w$, $\omega_{C^{D}}$ est annulé par $\mathfrak{m}_{K, w}$. On en déduit que le morphisme

$$
\omega_{C^{D} \otimes \vartheta_{K, 1-w}}=\omega_{C^{D}, 1-w} \longrightarrow \omega_{G^{D}, 1-w}=\omega_{G^{D} \otimes \vartheta_{K, 1-w}}
$$

est nul. Il suffit alors d'invoquer la proposition 1 . 
Remarque 4. - Nous montrons dans la section 7 que $C$ est également un cran de la filtration de Harder-Narasimhan de G.

\subsection{Compatibilité à la dualité}

Proposition 11. - Supposons $p \neq 2$. Soit $G$ un groupe de Barsotti-Tate tronqué d'échelon 1, de hauteur $h$ et de dimension $d$ sur $\theta_{K}$ tels que $0<d<h$. Supposons vérifiée l'inégalité $\mathrm{Ha}(G)<\frac{1}{2}$ si $p \neq 2$ et $\mathrm{Ha}(G)<\frac{1}{3}$ si $p=3$. Soient $C$ le sous-groupe de $G$ adhérence schématique de $\operatorname{ker} \alpha_{G, 1-\mathrm{Ha}(G)}$ et $D$ le sous-groupe de $G^{D}$ adhérence schématique de $\operatorname{ker} \alpha_{G^{D}, 1-\mathrm{Ha}(G)}$. On a alors l'égalité

$$
C\left(\theta_{K}\right)=D\left(\theta_{K}\right)^{\perp}
$$

Démonstration. - D'après la proposition $2, \mathrm{Ha}(G)=\mathrm{Ha}\left(G^{D}\right)$. On peut donc appliquer le théorème 4 à $G^{D}$ et $G$. On obtient ht $(D)=h-d$ et ht $(C)=d$. Il suffit alors de montrer que $D\left(\theta_{K}\right)^{\perp} \subset C\left(\theta_{K}\right)$. Notons $w=\mathrm{Ha}(G)$. Il y a une factorisation

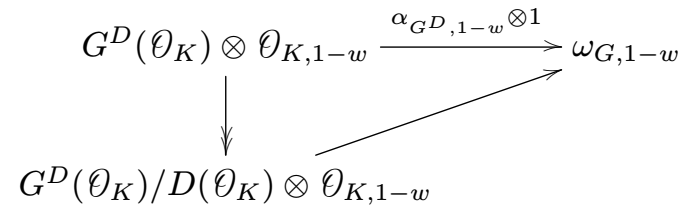

qui induit en passant au dual une factorisation

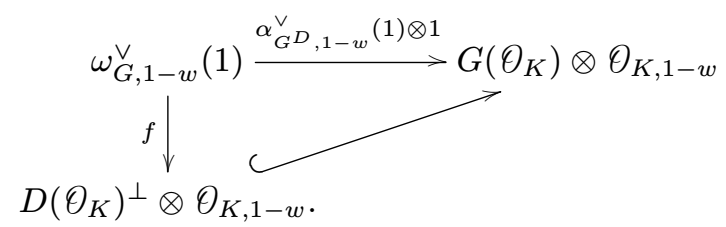

D'après le point (3) de la proposition 7 il existe un morphisme

$$
g: D\left(\theta_{K}\right)^{\perp} \otimes \vartheta_{K, 1-w} \longrightarrow \omega_{G, 1-w}^{\vee}(1)
$$

vérifiant $g \circ f=\delta$ Id où $\delta \in \vartheta_{K}$ est tel que $v(\delta)=\frac{w}{p-1}$. Considérons la suite de Hodge-Tate

$$
\omega_{G, 1-w}^{\vee}(1) \stackrel{\alpha_{G D, 1-w}^{\vee}(1) \otimes 1}{\longrightarrow} G\left(\oslash_{K}\right) \otimes \theta_{K, 1-w} \stackrel{\alpha_{G, 1-w} \otimes 1}{\longrightarrow} \omega_{G^{D}, 1-w} .
$$

D'après le théorème 2, la composée des deux applications dans la suite précédente est nulle (lorsque $K$ est le complété de la clôture algébrique d'un corps valué complet de valuation discrète cela est dû à Tate [34], ou bien à Fontaine [18]). Des considérations précédentes on déduit donc que

$$
\mathfrak{m}_{K, \frac{w}{p-1}} \cdot\left(D\left(\vartheta_{K}\right)^{\perp} \otimes \Theta_{K, 1-w}\right) \subset \operatorname{ker}\left(\alpha_{G, 1-w} \otimes 1\right) .
$$

On en déduit que $D\left(\theta_{K}\right)^{\perp} \subset \operatorname{ker} \alpha_{G^{D}, 1-w-\frac{w}{p-1}}=C\left(\theta_{K}\right)$ car $1-w-\frac{w}{p-1}>\frac{w}{p-1}$ (la proposition 10 s'applique donc avec $\epsilon=1-w-\frac{w}{p-1}$ ). 
Corollaire 1. - Supposons $p \neq 2$. Soit $G$ un groupe de Barsotti-Tate tronqué d'échelon 1 et de hauteur $h>0$ sur $\theta_{K}$. Supposons-le muni d'un isomorphisme $\lambda: G \stackrel{\sim}{\longrightarrow} G^{D}$ vérifiant $\lambda^{D}=\epsilon \lambda$ pour un $\epsilon \in \mathbb{Z}_{p}^{\times}$. Cela définit un accouplement parfait $G\left(\theta_{K}\right) \times G\left(\theta_{K}\right) \rightarrow \mathbb{F}_{p}(1)$. Supposons vérifiée l'inégalité $\mathrm{Ha}(G)<\frac{1}{2}$ si $p \neq 3$ et $\mathrm{Ha}(G)<\frac{1}{3}$ si $p=3$. Soit $C$ le sous-groupe de $G$ adhérence schématique de $\operatorname{ker} \alpha_{G, 1-\mathrm{Ha}(G)}$. Le groupe $C\left(\theta_{K}\right)$ est alors un sous-espace totalement isotrope maximal de $G\left(\theta_{K}\right)$.

Corollaire 2. - Supposons $p \neq 2$. Soit $G$ un groupe de Barsotti-Tate tronqué d'échelon 1 , de hauteur $h$ et de dimension d vérifiant $0<d<h$. Supposons vérifiée l'inégalité $\mathrm{Ha}(G)<\frac{1}{2}$ si $p \neq 3$ et $\mathrm{Ha}(G)<\frac{1}{3}$ si $p=3$. Alors, si $\lambda \in v\left(K^{\times}\right)$vérifie $\frac{\mathrm{Ha}(G)}{p-1} \leq \lambda<\frac{p}{p-1}(1-\mathrm{Ha}(G))$, les groupes $G_{\mathrm{AS}}^{\lambda}$ et $\left(G^{D}\right)_{\mathrm{AS}}^{\lambda}$ sont de hauteur d et $h-d$. De plus $G_{\mathrm{AS}}^{\lambda}\left(\theta_{K}\right)=\left(G^{D}\right)_{\mathrm{AS}}^{\lambda}\left(\theta_{K}\right)^{\perp}$. En particulier, si $G$ est muni d'un isomorphisme $\lambda: G \stackrel{\sim}{\longrightarrow} G^{D}$ tel que $\lambda^{D}=\epsilon \lambda$ pour un $\epsilon \in \mathbb{Z}_{p}^{\times}$, alors $G_{\mathrm{AS}}^{\lambda}\left(\theta_{K}\right)$ est un sous-espace totalement isotrope maximal de $G\left(\theta_{K}\right)$ relativement $\grave{a}$ l'accouplement défini par $\lambda$.

\section{Une borne effective pour la filtration des points de $p^{n}$-torsion}

Dans cette section on suppose que $K$ est algébriquement clos, ce qui est suffisant pour nos besoins.

\subsection{Un critère de liberté}

Le lemme qui suit ne pose pas de problème.

Lemme 10. - Soient $n \geq 1$ et $d \geq 1$ des nombres entiers. Soit $M$ un $\mathbb{Z} / p^{n} \mathbb{Z}$-module de longueur nd. Alors, pour tout entier $k$ vérifiant $1 \leq k<n$ on a $\operatorname{long}\left(p^{k} M\right) \leq(n-k) d$. De plus, $M$ est libre si et seulement si les inégalités précédentes sont des égalités pour tout $k$ vérifiant $1 \leq k<n$.

Proposition 12. - Soit $n \geq 1$ un entier. Soit $G$ un groupe de Barsotti-Tate tronqué d'échelon $n$ et de dimension d sur $\Theta_{K}$. Soit $C$ un sous-groupe plat fini de $G$ de hauteur $n d$. Supposons que

$$
\operatorname{deg}(C)>n d-1 .
$$

Le $\mathbb{Z} / p^{n} \mathbb{Z}$-module $C\left(\theta_{K}\right)$ est alors libre de rang $d$.

Démonstration. - Soit $k$ un entier vérifiant $1 \leq k<n$. Notons $E$ l'adhérence schématique dans $G$ de $C\left(\theta_{K}\right)\left[p^{k}\right]$ et $F$ celle de $p^{k} C\left(\theta_{K}\right)$. Il y a une suite de groupes plats finis

$$
0 \longrightarrow E \longrightarrow C \stackrel{\times p^{k}}{\longrightarrow} F \longrightarrow 0
$$

telle que $E$ soit un sous-groupe fermé de $C$ et la suite devienne exacte en fibre générique. D'après le corollaire 3 de [16], cela implique l'inégalité

$$
\operatorname{deg}(C) \leq \operatorname{deg}(E)+\operatorname{deg}(F) .
$$

Puisque $E$ est un sous-groupe de $G\left[p^{k}\right], \operatorname{deg}(E) \leq \operatorname{deg}\left(G\left[p^{k}\right]\right)=k d$. On a de plus $\operatorname{deg}(F) \leq \operatorname{ht}(F)([16]$ corollaire 2). On a donc

$$
n d-1<\operatorname{deg}(C) \leq k d+\operatorname{ht}(F) .
$$


On obtient donc

$$
\operatorname{ht}(F)>(n-k) d-1
$$

et donc d'après le lemme $10, \operatorname{ht}(F)=(n-k) d$. Cela étant vrai pour tout $k$, d'après le lemme $10 C\left(\theta_{K}\right)$ est un module libre.

\subsection{Sur l'application de Hodge-Tate des groupes de Barsotti-Tate tronqués}

Dans cette section nous étendons les résultats de la section 6.4 qui concernaient les groupes de Barsotti-Tate tronqués d'échelon 1 à ceux d'échelon quelconque.

Proposition 13. - Soit $G$ un groupe de Barsotti-Tate tronqué d'échelon $n$ sur $\emptyset_{K}$. Pour $\epsilon \in v\left(K^{\times}\right)$vérifiant $0 \leq \epsilon<1-\frac{1}{p-1}$, ker $\alpha_{G, n-\epsilon}$ est engendré par moins de d éléments. En d'autres termes, $\operatorname{ker} \alpha_{G, n-\epsilon} \simeq \underset{i=1}{\oplus} \mathbb{Z} / p^{a_{i}} \mathbb{Z}$ pour des entiers $\left(a_{i}\right)_{1 \leq i \leq d}$ vérifiant $0 \leq a_{i} \leq n$.

Démonstration. - Notons $h$ la hauteur de $G$ et $d$ sa dimension. Après choix d'une base de $\omega_{G^{D}}$, la suite exacte

$$
0 \longrightarrow \omega_{G[p]^{D}} \longrightarrow \omega_{G^{D}} \longrightarrow \omega_{G\left[p^{n-1}\right]^{D}} \longrightarrow 0
$$

déduite de la suite exacte $0 \rightarrow G[p] \rightarrow G \stackrel{\times p}{\longrightarrow} G\left[p^{n-1}\right] \rightarrow 0$, s'identifie à la suite

$$
0 \longrightarrow\left(p^{n-1} \theta_{K} / p^{n} \Theta_{K}\right)^{h-d} \longrightarrow\left(\theta_{K} / p^{n} \theta_{K}\right)^{h-d} \longrightarrow\left(\theta_{K} / p^{n-1} \theta_{K}\right)^{h-d} \longrightarrow 0 .
$$

On en déduit que le morphisme $\omega_{G[p]^{D}} \rightarrow \omega_{G^{D}}$ induit une injection $\omega_{G[p]^{D}, 1-\epsilon} \hookrightarrow \omega_{G^{D}, n-\epsilon}$. Celle-ci s'inscrit dans un diagramme commutatif

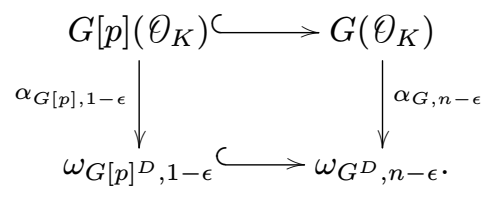

Soient $V=\operatorname{Im}\left(\alpha_{G, n-\epsilon}\right)$ et $V^{\prime}=\operatorname{Im}\left(\alpha_{G[p], 1-\epsilon}\right) \subset V$. Puisque $G[p]\left(\theta_{K}\right)=p^{n-1} G\left(\theta_{K}\right)$, $V^{\prime}=p^{n-1} V$. D'après la proposition $9, \operatorname{dim}_{\mathbb{F}_{p}} V^{\prime} \geq h-d$. On en déduit donc que

$$
\operatorname{dim}_{\mathbb{F}_{p}} p^{n-1} V \geq h-d .
$$

Si $V \simeq \bigoplus_{i=1}^{r} \mathbb{Z} / p^{b_{i}} \mathbb{Z}$ pour des entiers $\left(b_{i}\right)_{i}$ vérifiant $1 \leq b_{i} \leq n$, on a donc que $\left|\left\{i \mid b_{i}=n\right\}\right| \geq h-d$. De cela on déduit l'existence d'une décomposition $V=M_{1} \oplus M_{2}$, où $M_{1}$ est un $\mathbb{Z} / p^{n} \mathbb{Z}$-module libre de rang $h-d$. Le morphisme surjectif de $\mathbb{Z} / p^{n} \mathbb{Z}$-modules libres

$$
G\left(\vartheta_{K}\right) \stackrel{\alpha_{G, n-\epsilon}}{\longrightarrow} V \stackrel{\text { proj }}{\longrightarrow} M_{1}
$$

possède alors une section qui fournit un isomorphisme

$$
G\left(\theta_{K}\right) \simeq M_{1} \oplus N
$$

via lequel, le morphisme

$$
M_{1} \oplus N \simeq G\left(\theta_{K}\right) \stackrel{\alpha_{G, n-\epsilon}}{\longrightarrow} V=M_{1} \oplus M_{2}
$$

a une matrice de la forme

$$
\left(\begin{array}{cc}
\operatorname{Id}_{M_{1}} & 0 \\
* & *
\end{array}\right) .
$$

$4^{\text {e }}$ SÉRIE - TOME $44-2011-\mathrm{N}^{\circ} 6$ 
On en déduit que l'application composée

$$
\operatorname{ker}\left(\alpha_{G, n-\epsilon}\right) \hookrightarrow G\left(\theta_{K}\right) \simeq M_{1} \oplus N \stackrel{\text { proj }}{\longrightarrow} N
$$

est injective. Pour conclure, il suffit donc de montrer que $N$ est engendré par $d$ éléments. Or l'isomorphisme $G\left(\theta_{K}\right) \simeq M_{1} \oplus N$ induit un isomorphisme $G\left(\theta_{K}\right) / p G\left(\theta_{K}\right) \simeq M_{1} / p M_{1} \oplus N / p N$ où $\operatorname{dim}_{\mathbb{F}_{p}}\left(G\left(\theta_{K}\right) / p G\left(\theta_{K}\right)\right)=h$ et $\operatorname{dim}_{\mathbb{F}_{p}}\left(M_{1} / p M_{1}\right)=h-d$. On en déduit que $\operatorname{dim}_{\mathbb{F}_{p}}(N / p N)=d$ et donc $N$ est engendré par $d$ éléments.

\subsection{Sur la filtration de Harder-Narasimhan d'un groupe de Barsotti-Tate tronqué}

Proposition 14. - Soit G un groupe de Barsotti-Tate tronqué d'échelon n, de dimension $d$ et de hauteur $h$ sur $\vartheta_{K}$. Supposons qu'il existe un sous-groupe plat fini $C$ de $G$ tel que

$$
\operatorname{ht}(\mathrm{C})=n d \text { et } \operatorname{deg}(G / C)<1-\frac{1}{p-1} .
$$

Les propriétés suivantes sont alors vérifiées :

1. Si $\epsilon=\operatorname{deg}(G / C), C\left(\oslash_{K}\right)=\operatorname{ker}\left(\alpha_{G, n-\epsilon}\right)$ qui est un $\mathbb{Z} / p^{n} \mathbb{Z}$-module libre de rang $d$.

2. Si $C^{\prime}$ est un cran de la filtration de Harder-Narasimhan de $G$ vérifiant $\operatorname{ht}\left(C^{\prime}\right) \leq n d$ alors $C^{\prime} \subset C$.

3. Si $C^{\prime \prime}$ est un cran de la filtration de Harder-Narasimhan de $G$ vérifiant $\mathrm{ht}\left(C^{\prime \prime}\right) \geq n d$ alors $C \subset C^{\prime \prime}$.

Démonstration. - Commençons par le point (1). Puisque ht $(C)=\operatorname{deg}(G), \operatorname{deg}(G / C)=$ $\operatorname{deg}\left(\omega_{C^{D}}\right)$. On en déduit que le sous-module $\omega_{C^{D}}$ de $\omega_{G^{D}}$ est annulé par $\mathfrak{m}_{K, \epsilon}$. L'application composée $\omega_{C^{D}} \rightarrow \omega_{G^{D}} \rightarrow \omega_{G^{D}, n-\epsilon}$ est donc nulle. Il en résulte que $C\left(\theta_{K}\right) \subset \operatorname{ker}\left(\alpha_{G, n-\epsilon}\right)$. Les propositions 13 et 12 permettent alors de conclure quant au point (1).

Considérons maintenant le point (2). Puisque les pentes du polygone de HarderNarasimhan de $G$ sont comprises entre 0 et 1 , la fonction

$$
\begin{aligned}
{[0, n d] } & \longrightarrow \mathbb{R}_{+} \\
x & \longmapsto x-\mathrm{HN}(G)(x)
\end{aligned}
$$

est décroissante. On a donc l'inégalité

$$
\begin{aligned}
\operatorname{deg}\left(\omega_{C^{\prime D}}\right)=\operatorname{ht}\left(C^{\prime}\right)-\operatorname{deg}\left(C^{\prime}\right) & =\operatorname{ht}\left(C^{\prime}\right)-\operatorname{HN}(G)\left(\operatorname{ht}\left(C^{\prime}\right)\right) \\
& \leq \operatorname{ht}(C)-\operatorname{HN}(G)(\operatorname{ht}(C)) \\
& =\operatorname{deg}(G)-\operatorname{HN}(G)(\operatorname{ht}(C)) \\
& \leq \operatorname{deg}(G / C) .
\end{aligned}
$$

On en déduit comme dans le point (1) que $C^{\prime}\left(\theta_{K}\right) \subset \operatorname{ker}\left(\alpha_{G, n-\epsilon}\right)=C\left(\theta_{K}\right)$ et donc $C^{\prime} \subset C$.

Le point (3) résulte du point (2) appliqué à $G^{D}$. En effet, si $D \subset G^{D}$ désigne le sousgroupe plat fini tel que $D\left(\theta_{K}\right)=C\left(\theta_{K}\right)^{\perp}$ via l'accouplement parfait $G\left(\theta_{K}\right) \times G^{D}\left(\theta_{K}\right) \rightarrow$ $\mathbb{Q}_{p} / \mathbb{Z}_{p}(1)$, on a $\operatorname{deg}\left(G^{D} / D\right)=\operatorname{deg}(G / C)$. On peut alors appliquer le corollaire 8 de la section 5.1 de [16] pour conclure. 
Proposition 15. - Soit G un groupe de Barsotti-Tate tronqué d'échelon n et de dimension $d$ sur $\theta_{K}$. Supposons qu'il existe un sous-groupe $C$ de $G$ tel que $\operatorname{ht}(C)=$ nd et $\operatorname{deg}(G / C)<\frac{1}{2}$. Alors, $C$ est un cran de la filtration de Harder-Narasimhan de G. De plus, $C\left(\theta_{K}\right)$ est un $\mathbb{Z} / p^{n} \mathbb{Z}$-module libre de rang d et l'adhérence schématique de $C\left(\theta_{K}\right)^{\perp}$ dans $G^{D}$ est un cran de la filtration de Harder-Narasimhan de $G^{D}$.

Démonstration. - On vérifie sur la figure 1 que la condition implique bien que le polygone de Harder-Narasimhan de $G$ a un point de rupture en l'abscisse $n d$. D'après le point (2) de la proposition 14, ce cran coïncide avec le groupe $C$. La proposition 12 permet alors de conclure quant à l'assertion de liberté. Enfin les résultats de la section 5.1 de [16] montrent la compatibilité à la dualité.

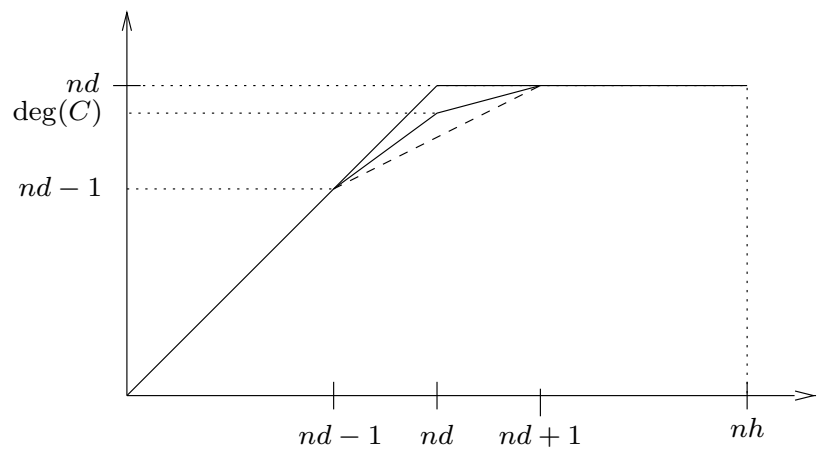

FIGURE 1. Une condition suffisante pour que $\operatorname{HN}(G)$ ait un point de rupture en l'abscisse $d$.

Remarque 5. - Dans la section 4.12 de [16] on réindexe la filtration de HarderNarasimhan de $G$ en une filtration décroissante $\left(G_{\mathrm{HN}}^{\lambda}\right)_{0 \leq \lambda \leq 1}$. La proposition précédente peut se reformuler en disant que le cran $G_{\mathrm{HN}}^{\frac{1}{2}}$ est tel que $G_{\mathrm{HN}}^{\frac{1}{2}}\left(\theta_{K}\right)$ soit libre de rang $d$ sur $\mathbb{Z} / p^{n} \mathbb{Z}$.

\subsection{Variation de l'invariant de Hodge sous l'isogénie canonique}

THÉORÈme 5. - Soit $G$ un groupe de Barsotti-Tate tronqué d'échelon 2 sur $\theta_{K}$. Supposons que $\mathrm{Ha}(G)<\frac{1}{p+1}$. Soit $C$ le sous-groupe canonique de $G[p]$ construit dans le théorème 4. Le groupe de Barsotti-Tate tronqué d'échelon $1, p^{-1} C / C$, vérifie

$$
\mathrm{Ha}\left(p^{-1} C / C\right)=p \mathrm{Ha}(G) .
$$

De plus, si $\frac{1}{p+1} \leq \mathrm{Ha}(G)<\frac{1}{2}$, alors

$$
\mathrm{Ha}\left(p^{-1} C / C\right) \geq 1-\mathrm{Ha}(G) .
$$

Démonstration. - Soient $S$ un schéma annulé par $p$ et $H$ un groupe de Barsotti-Tate tronqué d'échelon 2 sur $S$. Soit $F$ le morphisme de Frobenius de $H$. Le groupe plat fini $E=p^{-1} \operatorname{ker} F / \operatorname{ker} F$ est un groupe de Barsotti-Tate tronqué d'échelon 1 et il y a un isomorphisme

$$
F: E \stackrel{\sim}{\longrightarrow} G[p]^{(p)} .
$$


Celui-ci induit un isomorphisme $\omega_{E} \stackrel{\sim}{\longrightarrow} \omega_{G}^{(p)}$ et donc

$$
\operatorname{det}\left(\omega_{E}\right) \stackrel{\sim}{\longrightarrow} \operatorname{det}\left(\omega_{G}\right)^{\otimes p} .
$$

On vérifie aussitôt que via cette identification on a

$$
\widetilde{\mathrm{Ha}}(E)=\widetilde{\mathrm{Ha}}(G)^{\otimes p} \text {. }
$$

Notons maintenant $w=\mathrm{Ha}(G)$. D'après le point (3) du théorème 4 la réduction sur $\theta_{K, 1-w}$ de $C$ coïncide avec le noyau du Frobenius de $G$. On a donc

$$
\inf \{p w, 1-w\}=\inf \left\{\mathrm{Ha}\left(p^{-1} C / C\right), 1-w\right\} .
$$

Le résultat annoncé se déduit de cette égalité.

Comme conséquence des théorèmes 4 et 5 on obtient le corollaire qui suit.

Corollaire 3. - Supposons $p \neq 2,3$. Soit $H$ un groupe $p$-divisible non-ordinaire sur $\theta_{K}$. Il existe alors un groupe $p$-divisible $H^{\prime}$ sur $\theta_{K}$ isogène à $H$ et tel que

$$
\mathrm{Ha}\left(H^{\prime}\right) \geq \frac{1}{2}
$$

Remarque 6. - Dans cette section nous supposons $K$ algébriquement clos. Notons néanmoins que si ce n'est pas le cas, le corollaire précédent reste valable; le groupe $p$-divisible $H^{\prime}$ peut être pris défini sur $\theta_{K}$.

\subsection{Le théorème final}

ThÉORÈme 6. - Supposons $p \neq 2$. Soient $0 \leq d<h$ des entiers. Soit $G$ un groupe de Barsotti-Tate tronqué d'échelon $n$, de hauteur $h$ et de dimension $d$ sur $\theta_{K}$ tel que

$$
\mathrm{Ha}(G)<\frac{1}{2 p^{n-1}},
$$

si $p \neq 3$ et $\mathrm{Ha}(G)<\frac{1}{3^{n}}$ si $p=3$.

1. La filtration de Harder-Narasimhan de $G$ possède alors un cran $C$ tel que $C\left(\theta_{K}\right)$ soit un $\mathbb{Z} / p^{n} \mathbb{Z}$-module libre de rang $d$.

2. La formule suivante est vérifiée : $\operatorname{deg}(G / C)=\frac{p^{n}-1}{p-1} \mathrm{Ha}(G)$.

3. Si pour $1 \leq k<n$ si l'on note $C_{k}$ l'adhérence schématique de $C\left(\vartheta_{K}\right)\left[p^{k}\right]$ dans $G, C_{k}$ est le cran de hauteur $k d$ de la filtration de Harder-Narasimhan de $G\left[p^{k}\right]$.

4. Pour $1 \leq k<n$ considérons $p^{-(n-k)} C_{k} / C_{k}$, un groupe de Barsotti-Tate tronqué d'échelon $n-k$. Alors $\mathrm{Ha}\left(p^{-(n-k)} C_{k} / C_{k}\right)=p^{k} \mathrm{HaG}$ et $C / C_{k}$ est le cran de la filtration de Harder-Narasimhan de $p^{-(n-k)} C_{k} / C_{k}$ de hauteur $(n-k) d$.

5. La filtration de Harder-Narasimhan de $G^{D}$ possède également un cran dont les $\vartheta_{K}$-points forment un $\mathbb{Z} / p^{n} \mathbb{Z}$-module libre de rang $(h-d)$ et ce cran est égal à l'orthogonal $d u$ précédent via l'accouplement parfait $G\left(\theta_{K}\right) \times G^{D}\left(\theta_{K}\right) \rightarrow \mathbb{Z} / p^{n} \mathbb{Z}(1)$.

6. Pour $1 \leq k \leq n$ le sous-groupe $C_{k} \otimes \bigoplus_{K, 1-p^{k-1} \mathrm{Ha}(G)}$ de $G \otimes \bigoplus_{K, 1-p^{k-1} \mathrm{Ha}(G)}$ coüncide avec le noyau du k-ième itéré de Frobenius.

7. Le groupe $C\left(\theta_{K}\right)$ coüncide avec le noyau de l'application de Hodge-Tate $\alpha_{G, n-\frac{p^{n}-1}{p-1} \mathrm{Ha}(G)}$. 
Démonstration. - On suppose $p \neq 2$, le cas $p=3$ étant laissé au lecteur. On procède par récurrence sur $n$, le cas $n=1$ étant une conséquence du théorème 4 . Supposons donc l'hypothèse vérifiée pour les groupes d'échelon $n-1$ avec $n \geq 2$. Soit $G$ un groupe d'échelon $n$ vérifiant les hypothèses de l'énoncé. Le groupe $G[p]$ satisfait en particulier aux hypothèses du théorème 4 et de la proposition 15. Soit donc $D \subset G[p]$ le sous-groupe fourni par le théorème 4. Le groupe plat fini $p^{-1} D / D$ est un groupe de Barsotti-Tate tronqué d'échelon 1 , de hauteur $h$ et de dimension $d$. Puisque $n \geq 2, G\left[p^{2}\right]$ satisfait aux hypothèses du théorème 5 qui donne donc l'égalité

$$
\mathrm{Ha}\left(p^{-1} D / D\right)=p \mathrm{Ha}(G) .
$$

On peut donc appliquer l'hypothèse de récurrence au groupe de Barsotti-Tate tronqué d'échelon $n-1, p^{-(n-1)} C / C$. Soit donc $C$ tel que $D \subset C \subset p^{-(n-1)} D$ et $C / D$ soit le sous-groupe donné par l'hypothèse de récurrence. D'après le point (1) du théorème 4, $\operatorname{deg}(G[p] / D)=\mathrm{Ha}(G)$. On a l'égalité

$$
\begin{aligned}
\operatorname{deg}(G / C) & =\operatorname{deg}\left(G / p^{-(n-1)} D\right)+\operatorname{deg}\left(p^{-(n-1)} D / C\right) \\
& =\operatorname{deg}\left(G / p^{-(n-1)} D\right)+\frac{p^{n-1}-1}{p-1} p \operatorname{Ha}(G) .
\end{aligned}
$$

Or, il y a un isomorphisme

$$
\times p^{n-1}: G / p^{-(n-1)} D \stackrel{\sim}{\longrightarrow} G[p] / D .
$$

On obtient donc $\operatorname{deg}(G / C)=\frac{p^{n}-1}{p-1} \mathrm{Ha}(G)$, c'est-à-dire le point (2) de l'énoncé au cran $n$.

La difficulté réside maintenant à montrer que $C$ est un cran de la filtration de HarderNarasimhan de $G$. En effet, on aimerait appliquer la proposition 15 mais malheureusement $\frac{p^{n}-1}{p-1} \times \frac{1}{2 p^{n-1}}$ n'est pas strictement plus petit que $\frac{1}{2}$. Néanmoins on vérifie que

$$
\frac{1}{2 p^{n-1}} \times \frac{p^{n}-1}{p-1}<1-\frac{1}{p-1}
$$

et donc d'après la proposition 14 il suffit de montrer que le polygone de Harder-Narasimhan de $G$ possède un point de rupture en l'abscisse $n d$. Notons $\mathscr{P}=\mathrm{HN}(G)$ ce polygone. Il suffit de montrer que, pour des entiers $i, j$ vérifiant $0 \leq i<n d<j \leq n h$ et tels que $i$ et $j$ soient des abscisses de points de rupture de $\mathscr{P}$, l'inégalité suivante est vérifiée :

$$
\frac{n d-i}{j-i} \mathscr{P}(j)+\frac{j-n d}{j-i} \mathscr{P}(i)<n d-\frac{p^{n}-1}{p-1} \mathrm{Ha}(G) .
$$

Commençons par vérifier que c'est le cas si $i \leq n d-2$. Rappelons que si $0 \leq x \leq n d$ alors $\mathscr{P}(x) \leq x$ et pour tout $x, \mathscr{P}(x) \leq n h$. De cela et de l'inégalité $i \leq n d-2$ on déduit en raisonnant sur un dessin analogue à la figure 1 que

$$
\frac{n d-i}{j-i} \mathscr{P}(j)+\frac{j-n d}{j-i} \mathscr{P}(i) \leq n d-\frac{2}{3}
$$

Or, on a

$$
\frac{1}{2 p^{n-1}} \times \frac{p^{n}-1}{p-1}<\frac{2}{3}
$$

Cela fournit l'inégalité (6) lorsque $i \leq n d-2$. On vérifie de la même façon cette inégalité lorsque $j \geq n d+2$. 
Reste donc le cas où $i=n d-1$ et $j=n d+1$. Notons $w=\mathrm{Ha}(G)$. Soit donc $A$ un sous-groupe de $G$ de hauteur $n d-1$ tel que $A$ soit un cran de la filtration de HarderNarasimhan de $G$. D'après la proposition 14, $A \subset C$. Puisque $C\left(\theta_{K}\right) \simeq\left(\mathbb{Z} / p^{n} \mathbb{Z}\right)^{d}$, $A\left(\theta_{K}\right) \simeq\left(\mathbb{Z} / p^{n} \mathbb{Z}\right)^{d-1} \oplus\left(\mathbb{Z} / p^{n-1} \mathbb{Z}\right)$. Notons $A\left[p^{n-1}\right]$ l'adhérence schématique de $A\left(\theta_{K}\right)\left[p^{n-1}\right]$ et $p^{n-1} A$ celle de $p^{n-1} A\left(\theta_{K}\right)$. D'après le corollaire 3 de [16], la suite $0 \rightarrow A\left[p^{n-1}\right] \rightarrow A \rightarrow p^{n-1} A \rightarrow 0$, exacte en fibre générique, fournit l'inégalité

$$
\operatorname{deg}(A) \leq \operatorname{deg}\left(A\left[p^{n-1}\right]\right)+\operatorname{deg}\left(p^{n-1} A\right) .
$$

On a de plus $A\left[p^{n-1}\right]=C_{n-1}$, le sous-groupe canonique de niveau $n-1$, et $\mathrm{ht}\left(p^{n-1} A\right)=d-1$. L'hypothèse de récurrence au cran $n-1$ nous donne donc $\operatorname{deg}\left(A\left[p^{n-1}\right]\right)=(n-1) d-\frac{p^{n-1}-1}{p-1} w$. De cela on déduit que

$$
\operatorname{deg}(A) \leq n d-1-\frac{p^{n-1}-1}{p-1} w .
$$

Maintenant si $B$ est un sous-groupe plat fini de $G$ qui est un cran de sa filtration de HarderNarasimhan de hauteur $n d+1$, par application du raisonnement précédent à $G^{D}$ (qui satisfait aux même hypothèses que $G$ ) et du corollaire 8 de [16], on obtient

$$
\operatorname{deg}(B) \leq n d-\frac{p^{n-1}-1}{p-1} w .
$$

Plus précisément, $(G / B)^{D}$ est un cran de la filtration de Harder-Narasimhan de $G^{D}$ de hauteur $n(h-d)-1$ et de degré $n(h-d)-(n d+1)+\operatorname{deg} B$. Le groupe de Barsotti-Tate tronqué $G^{D}$ est de hauteur $h$ et de dimension $h-d$ et vérifie $\mathrm{Ha}(G)=\mathrm{Ha}\left(G^{D}\right)$. On a donc par application du raisonnement précédent à $G^{D}$ et $B$,

$$
\operatorname{deg}(G / B)^{D} \leq n(h-d)-1-\frac{p^{n-1}-1}{p-1} w,
$$

inégalité de laquelle on déduit celle annoncée pour $\operatorname{deg}(B)$.

Il suffit maintenant de vérifier que si $w=\mathrm{Ha}(G)$, alors

$$
\frac{1}{2}\left(n d-1-\frac{p^{n-1}-1}{p-1} w\right)+\frac{1}{2}\left(n d-\frac{p^{n-1}-1}{p-1} w\right)<n d-\frac{p^{n}-1}{p-1} w
$$

ou encore que

$$
\left(\frac{p^{n}-1}{p-1}-\frac{p^{n-1}-1}{p-1}\right) w<\frac{1}{2}
$$

ce qui résulte de l'hypothèse faite sur $w$.

Les autres points de l'énoncé s'obtiennent aisément (il faut bien sûr invoquer le théorème 5 pour le point (6)).

\subsection{Isogénies anticanoniques}

Proposition 16. - Soit $H$ un groupe $p$-divisible sur $\oslash_{K}$ tel que $\mathrm{Ha}(H)<\frac{p-2}{2 p-2}$. Il existe alors un groupe $p$-divisible $H^{\prime}$ sur $\vartheta_{K}$ vérifiant $\mathrm{Ha}\left(H^{\prime}\right)=\frac{1}{p} \mathrm{Ha}(H)$ et $H=H^{\prime} / C^{\prime}$ où $C^{\prime} \subset H^{\prime}[p]$ est le sous-groupe canonique déduit du théorème 4, c'est-à-dire le cran de hauteur $\operatorname{dim} H$ de la filtration de Harder-Narasimhan de $H[p]$. 
Démonstration. - Puisque $\mathrm{Ha}(H)<\frac{1}{2}$ le théorème 4 s'applique. Soit donc $C \subset H[p]$ le sous-groupe fourni par ce théorème. Soit $D \subset H[p]$ un sous-groupe plat fini tel que $H[p]\left(\vartheta_{K}\right)=C\left(\vartheta_{K}\right) \oplus D\left(\theta_{K}\right)$ (rappelons que l'on suppose que $K$ est algébriquement clos, il existe donc toujours un tel $D$ ). Posons $H^{\prime}=H / D$ et $C^{\prime}=H[p] / D$. Il y a un morphisme naturel $C \rightarrow C^{\prime}$ qui est un isomorphisme en fibre générique. Cela induit un diagramme commutatif

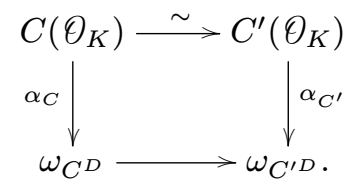

Posons $w=\mathrm{Ha}(G)$. On a $C\left(\theta_{K}\right)=\operatorname{ker} \alpha_{H[p], 1-w}$ (cf. théorème 4). On en déduit donc que $\alpha_{C^{\prime}, 1-w}=0$. D'après le théorème 3 , cela implique que $\mathfrak{m}_{K, \frac{1}{p-1}+w} \cdot \omega_{C^{\prime D}}=0$. On en déduit que le morphisme

$$
\omega_{C^{\prime D}, 1-\frac{1}{p-1}-w} \longrightarrow \omega_{H^{\prime}[p]^{D}, 1-\frac{1}{p-1}-w}
$$

est nul. D'après la proposition 1 cela implique que $C^{\prime} \otimes \vartheta_{K, 1-\frac{1}{p-1}-w}$ coïncide avec le noyau du morphisme de Frobenius de $H^{\prime}[p] \otimes \vartheta_{K, 1-\frac{1}{p-1}-w}$. Comme dans la démonstration du théorème 5 , on en déduit que

$$
\inf \{\underbrace{\mathrm{Ha}\left(H^{\prime} / C^{\prime}\right)}_{w}, 1-\frac{1}{p-1}-w\}=\inf \left\{p \mathrm{Ha}\left(H^{\prime}\right), 1-\frac{1}{p-1}-w\right\} .
$$

L'inégalité $w<1-\frac{1}{p-1}-w$ implique donc que $p \mathrm{Ha}\left(H^{\prime}\right)=w$.

\section{Application aux familles et aux espaces de modules}

\subsection{Le théorème général pour les familles}

THÉORÈme 7. - Supposons $p \neq 2$. Soit $F \mid \mathbb{Q}_{p}$ une extension valuée complète de valuation discrète. Soit $\mathfrak{X}$ un $\operatorname{Spf}\left(\theta_{F}\right)$-schéma formel topologiquement de type fini sans p-torsion et réduit. Soit $G$ un groupe de Barsotti-Tate tronqué d'échelon $n$, de hauteur h et de dimension d telle que $0<d<h$. Posons

$$
\epsilon_{n}=\frac{1}{2 p^{n-1}}
$$

si $p \neq 3$ et $\epsilon_{n}=\frac{1}{3^{n}}$ si $p=3$. Soit $U=\mathfrak{X}_{\text {ord }}^{\text {rig }}\left(\epsilon_{n}^{\circ}\right)$ le voisinage tubulaire du lieu ordinaire où l'invariant de Hasse est strictement plus petit que $\epsilon_{n}$. Il existe alors un unique sous-groupe étale fini $\mathrm{Fil} G^{\mathrm{rig}}$ du groupe étale fini $G^{\mathrm{rig}}{ }_{\mid U}$ tel que :

- Fil $G^{\mathrm{rig}}$ est localement isomorphe pour la topologie étale au groupe constant $\left(\mathbb{Z} / p^{n} \mathbb{Z}\right)^{d}$.

- En tout point de U, la fibre de Fil $G^{\text {rig }}$ est un cran de la filtration de Harder-Narasimhan de la fibre de $G$.

La filtration précédente de $G^{\mathrm{rig}}$ est invariante sous $\operatorname{End}(G)$. Si de plus $G$ est muni d'un isomorphisme $\lambda: G \stackrel{\sim}{\longrightarrow} G^{D}$ vérifiant $\lambda^{D}=\epsilon \lambda$ pour un $\epsilon \in \mathbb{Z}_{p}^{\times}$alors Fil $G^{\text {rig }}$ est totalement isotrope de rang maximal relativement à l'accouplement $G^{\mathrm{rig}} \times G^{\mathrm{rig}} \longrightarrow \mathbb{Z} / p^{n} \mathbb{Z}(1)$ défini par $\lambda$.

Démonstration. - C'est une conséquence du théorème 6 et du théorème 4 de [16].

$4^{\mathrm{e}}$ SÉRIE - TOME $44-2011-\mathrm{N}^{\circ} 6$ 


\subsection{Application à certaines variétés de Shimura de type PEL}

8.2.1. - Soit $(G, X)$ une donnée de Shimura de type PEL ([11], [29]). On note $\overline{\mathbb{Q}}$ la clôture algébrique de $\mathbb{Q}$ dans $\mathbb{C}$. Notons $E$ le corps reflex associé, un corps de nombres dans $\overline{\mathbb{Q}}$. À une telle donnée est associée une tour de variétés de Shimura quasi-projectives lisses sur $E$ munie d'une action de $G\left(\mathbf{A}_{f}\right)$

$$
\left(\mathrm{Sh}_{K}\right)_{K \subset G\left(\mathbf{A}_{f}\right)}
$$

où $K$ parcourt des sous-groupes compacts ouverts «suffisamment petits» dans $G\left(\mathbf{A}_{f}\right)$. Il y a de plus une uniformisation complexe

$$
\operatorname{Sh}_{K}(\mathbb{C})=\coprod_{\operatorname{ker}^{1}(\mathbb{Q}, G)} G(\mathbb{Q}) \backslash\left(X \times G\left(\mathbf{A}_{f}\right) / K\right),
$$

où l'ensemble fini $\operatorname{ker}^{1}(\mathbb{Q}, G)$ paramètre l'obstruction au principe de Hasse pour $G$.

Soit maintenant $p$ un nombre premier tel que le groupe réductif $G_{\mathbb{Q}_{p}}$ soit non ramifié. On fixe une clôture algébrique $\overline{\mathbb{Q}}_{p}$ de $\mathbb{Q}_{p}$ ainsi qu'un plongement $\nu: \overline{\mathbb{Q}} \hookrightarrow \overline{\mathbb{Q}}_{p}$ qui détermine un complété $p$-adique $E_{\nu}$ de $E$. Soit $\mathfrak{C}$ un sous-groupe compact maximal hyperspécial dans $G\left(\mathbb{Q}_{p}\right)$. Il y a alors une tour modulaire de $\operatorname{Spec}\left(\theta_{E_{\nu}}\right)$-schémas quasi projectifs lisses munie d'une action de $G\left(\mathbf{A}_{f}^{p}\right)$ (cf. [29])

$$
\left(S_{K^{p}}\right)_{K^{p} \subset G\left(\mathbf{A}_{f}^{p}\right)}
$$

qui forme un modèle entier de la tour précédente en niveau $\mathfrak{C}$ en $p$. En d'autres termes, la tour précédente est munie d'un isomorphisme $G\left(\mathbf{A}_{f}^{p}\right)$-équivariant

$$
\left(S_{K^{p}} \otimes_{\vartheta_{E \nu}} E_{\nu}\right)_{K^{p} \subset G\left(\mathbf{A}_{f}^{p}\right)} \stackrel{\sim}{\longrightarrow}\left(\mathrm{Sh}_{\mathfrak{C} K^{p}} \otimes_{E} E_{\nu}\right)_{K^{p}} .
$$

Notons

$$
\phi_{K^{p}}:=\left(\widehat{S}_{K^{p}}\right)^{\text {rig }}
$$

l'espace analytique rigide fibre générique du complété $p$-adique de $S_{K^{p}}$. C'est un ouvert admissible quasicompact Hecke-invariant dans l'espace rigide $S_{\mathfrak{C} K^{p}}^{\text {rig }}$. Il s'agit du « lieu de bonne réduction » dans $\mathrm{Sh}_{\mathfrak{C} K^{p}}^{\text {rig }}$, par exemple si nos variétés de Shimura sont des courbes modulaires, il y a un morphisme $j$-invariant $\operatorname{Sh}_{K_{p} K^{p}} \stackrel{j}{\rightarrow} \mathbb{A}^{1}$ et $\oint_{K_{p} K^{p}}=\left(j^{\text {rig }}\right)^{-1}(\mathbb{B}(0,1))$. Pour un sous-groupe ouvert $K_{p} \subset \mathfrak{C}$, via le morphisme d'oubli du niveau $\mathrm{Sh}_{K_{p} K^{p}}^{\text {rig }} \rightarrow \mathrm{Sh}_{\mathfrak{C} K^{p}}^{\text {rig }}$, cela définit par image réciproque en niveau $K_{p}$ un espace rigide quasicompact $\oint_{K_{p} K^{p}}$. Il y a donc une tour $G\left(\mathbf{A}_{f}\right)$-équivariante d'espaces rigides

$$
\left(\phi_{K_{p} K^{p}}\right)_{K_{p} \subset \mathfrak{C}, K^{p}} .
$$

8.2.2. - On suppose maintenant pour simplifier que le groupe $G$ est un groupe de similitudes unitaires ou bien symplectiques (les cas (A) et (C) de [29]). Décomposons le groupe dérivé $G_{\mathbb{Q}_{p}}^{\text {der }}$ en facteurs simples,

$$
G_{\mathbb{Q}_{p}}^{\mathrm{der}}=\prod_{i \in I} G_{\mathbb{Q}_{p}, i}^{\mathrm{der}}
$$

Dans le cas (A) les groupes $G_{\mathbb{Q}_{p}, i}^{\text {der }}$ sont soit des restrictions des scalaires de groupes spéciaux linéaires soit des groupes spéciaux unitaires $p$-adiques. Dans le cas (C) ce sont des restrictions des scalaires de groupes symplectiques $p$-adiques. Soit $A$ le schéma abélien universel sur $S_{K^{p}}$ 
muni de sa polarisation principale en $p$. À la décomposition précédente est associée une décomposition du groupe $p$-divisible polarisé

$$
A\left[p^{\infty}\right]=\bigoplus_{i \in I} H_{i}
$$

où :

- Dans le cas (A) si $G_{\mathbb{Q}_{p}, i}^{\text {der }}$ est un groupe spécial linéaire alors $H_{i}=\mathcal{H}_{i} \oplus \mathcal{H}_{i}^{D}$ muni de la polarisation tautologique, $\mathcal{H}_{i}$ étant un groupe $p$-divisible muni d'une action de l'anneau des entiers d'une extension non ramifiée de $\mathbb{Q}_{p}$.

- Dans le cas (A) si $G_{\mathbb{Q}_{p}, i}^{\text {der }}$ est un groupe unitaire $H_{i}$ est un groupe $p$-divisible principalement polarisé, muni d'une action de l'anneau des entiers d'une extension non ramifiée de $\mathbb{Q}_{p}$ équipée d'une involution non triviale, de telle manière que l'involution de Rosati induite par la polarisation soit compatible à cette involution du corps $p$-adique.

- Dans le cas (C) $H_{i}$ est un groupe $p$-divisible principalement polarisé muni d'une action de l'anneau des entiers d'une extension non ramifiée de $\mathbb{Q}_{p}$, de telle manière que l'involution de Rosati agisse trivialement sur ce corps $p$-adique.

Fixons un $i \in I$ et soit $H$ le groupe $p$-divisible associé, $H=H_{i}$ dans le cas (A) unitaire ou le cas $(\mathrm{C})$ et $H=\mathscr{H}_{i}$ dans le cas (A) linéaire. Soit $\mu: \mathbb{G}_{m} \rightarrow G_{\overline{\mathbb{Q}}}$ un cocaractère de Hodge de la forme $\mu_{x}$ pour un $x \in X$. Via le plongement $\nu$ il définit un cocaractère de Hodge $p$-adique $\mu_{\overline{\mathbb{Q}}_{p}}: \mathbb{G}_{m} \longrightarrow G_{\overline{\mathbb{Q}}_{p}}$ bien défini à $G\left(\overline{\mathbb{Q}}_{p}\right)$-conjugaison près. Soit $\mu_{i}: \mathbb{G}_{m} \longrightarrow G_{\overline{\mathbb{Q}}_{p}, i, a d}$ la projection de ce cocaractère sur le groupe adjoint de $G_{\overline{\mathbb{Q}}_{p}, i}$, un cocaractère minuscule. Kottwitz a défini et étudié dans [28] un ensemble fini $B\left(G_{\mathbb{Q}_{p}, a d, i}, \mu_{i}\right)$ (on triche légèrement ici car en fait il ne faut pas prendre le groupe $G_{\mathbb{Q}_{p}, i}$ mais un certain groupe réductif de groupe dérivé $G_{\mathbb{Q}_{p}, i}$ et un certain relèvement de $\mu_{i}$ dans ce groupe) qui paramètre les différents polygones de Newton possibles pour la réduction modulo $p$ du groupe $p$-divisible muni de ses structures additionnelles $H$. Il y a toujours un polygone minimal dans cet ensemble qui définit un ouvert de la fibre spéciale de $S_{K^{p}}$ appelé lieu $\mu$-ordinaire. D'après [39] ce lieu est Zariski dense dans la fibre spéciale. Ce lieu ne coïncide par toujours avec le lieu d'ordinarité de $H$ qui peut être vide (s'il est non vide les deux coïncident bien sûr). Nous allons maintenant faire l'hypothèse suivante :

Hypothèse : Le lieu $\mu$-ordinaire de H correspond au lieu ordinaire au sens de la définition 1.

Cette hypothèse est toujours vérifiée dans le cas (C). C'est par exemple le cas des variétés de Siegel ou bien des variétés modulaires de Hilbert. On vérifie en général que cette hypothèse est vérifiée si et seulement si la classe de conjugaison de $\mu_{i}$ est définie sur $\mathbb{Q}_{p}$ ([39] 1.6.3).

8.2.3. - Soit $\mathfrak{G}$ un modèle entier réductif de $G$ tel que $\mathfrak{C}=\mathfrak{G}\left(\mathbb{Z}_{p}\right)$. À l'indice $i \in I$ précédent et au polygone de Newton ordinaire est associé un sous-groupe parabolique de $\mathfrak{G}$. On peut lui associer une famille décroissante de sous-groupes de congruence $\left(\mathfrak{P}_{n}\right)_{n \geq 0}$ du groupe compact hyperspécial $\mathfrak{C}$ où $\mathfrak{P}_{0}=\mathfrak{C}$. Le groupe $\mathfrak{P}_{1}$ est un sous-groupe parahorique et pour $n \geq 1$

$$
\mathfrak{P}_{n}=\left\{g \in \mathfrak{G}\left(\mathbb{Z}_{p}\right) \mid g \bmod p^{n} \in \mathfrak{P}\left(\mathbb{Z} / p^{n} \mathbb{Z}\right)\right\}
$$


EXemple 2. - Soit $\mathrm{GSp}_{2 g}$ le groupe de similitudes symplectiques associé à la matrice $\left(\begin{array}{cc}0 & -I_{g} \\ I_{g} & 0\end{array}\right)$. Considérons le cas où $G=\left\{x \in \operatorname{Res}_{F / \mathbb{Q}} \operatorname{GSp}_{2 g} \mid \operatorname{det}(x) \in \mathbb{Q}^{\times}\right\}$pour un corps totalement réel $F$. Le cas $F=\mathbb{Q}$ est celui des variétés de Siegel. Quant au cas $g=1$ il s'agit de celui des variétés modulaires de Hilbert. On a alors

$$
G_{\mathbb{Q}_{p}}=\left\{\left(x_{v}\right)_{v} \in \prod_{v \mid p} \operatorname{Res}_{F_{v} / \mathbb{Q}_{p}} \operatorname{Gsp}_{2 g} \mid \forall v_{1}, v_{2}, \operatorname{det}\left(x_{v_{1}}\right)=\operatorname{det}\left(x_{v_{2}}\right) \in \mathbb{Q}_{p}^{\times}\right\} .
$$

Le groupe $p$-divisible $A\left[p^{\infty}\right]$ se scinde en $A\left[p^{\infty}\right]=\underset{v \mid p}{\oplus} H_{v}$ où $H_{v}$ est muni d'une action de $\vartheta_{F_{v}}$ et d'une polarisation principale compatible à l'action de $\Theta_{F_{v}}$. On a de plus ht $H_{v}=2\left[F_{v}: \mathbb{Q}_{p}\right] g$ et $\operatorname{dim} H_{v}=\left[F_{v}: \mathbb{Q}_{p}\right] g$. Fixons $v_{0} \mid p$. Alors

$$
\begin{aligned}
\mathfrak{C} & =\left\{\left(x_{v}\right)_{v} \in \prod_{v \mid p} \operatorname{GSp}_{2 g}\left(\Theta_{F_{v}}\right) \mid \forall v_{1}, v_{2}, \operatorname{det}\left(x_{v_{1}}\right)=\operatorname{det}\left(x_{v_{2}}\right) \in \mathbb{Z}_{p}^{\times}\right\}, \\
\mathfrak{P}_{n} & =\left\{\left(x_{v}\right)_{v} \in \mathfrak{C} \mid x_{v_{0}} \equiv\left(\begin{array}{c}
* * \\
0 *
\end{array}\right) \bmod p^{n}\right\},
\end{aligned}
$$

les blocs précédents étant de taille $g \times g$.

Considérons la tour $G\left(\mathbf{A}_{f}^{p}\right)$-équivariante d'espaces rigides $\left(\phi_{\mathfrak{P}_{n} K^{p}}\right)_{n \geq 0, K^{p}}$. Afin d'alléger les notations oublions l'indice $K^{p}$ dans les notations précédentes. Pour $n \geq k \geq 0$, il y a une correspondance de Hecke

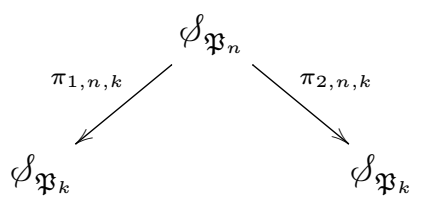

où $\pi_{1, n, k}$ est le morphisme d'oubli du niveau. Au niveau de la variété algébrique $\mathrm{Sh}_{\mathfrak{P}_{n} K^{p}}$ le morphisme $\pi_{2, n, k}$ envoie le couple $(A, C)$, où $A$ est une variété abélienne avec structures additionnelles et $C \subset H\left[p^{n}\right]$ un sous-groupe satisfaisant certaines propriétés, sur le couple $\left(A / C\left[p^{n-k}\right], C / C\left[p^{n-k}\right]\right)$.

ThÉorème 8. - Posons, pour $n \geq 1, \epsilon_{n}=\frac{1}{2 p^{n-1}}$. Pour $k \geq 0, \epsilon>0$, on note $\left(\phi_{\mathfrak{P}_{k}}\right)_{\text {ord }}(\stackrel{\bullet}{\epsilon})$ le tube du lieu ordinaire dans $\phi_{\mathfrak{P}_{k}}$ où l'invariant de Hasse est strictement plus petit que $\epsilon$.

1. Il y a alors pour tout $n \geq 1$ une section $s_{n}$

$$
\begin{gathered}
\left(\phi_{\mathfrak{P}_{n}}\right)_{\text {ord }}\left(\stackrel{\circ}{\epsilon}_{n}\right) \\
\pi_{1, n, 0} \downarrow \int^{\mid} s_{n} \\
\phi_{\text {ord }}\left(\stackrel{\circ}{\epsilon}_{n}\right)
\end{gathered}
$$

étendant la section canonique sur le lieu ordinaire.

2. Posons $U_{p}=\pi_{2,1,0} \circ s_{1}: \phi_{\text {ord }}\left(\frac{i}{2}\right) \rightarrow \&$ l'opérateur "quotient par le sous-groupe canonique ». En restriction au tube $\&_{\text {ord }}\left(\frac{\stackrel{1}{p}}{p+1}\right)$ on a l'égalité suivante de fonctions à valeurs dans $[0,1]$,

$$
\mathrm{Ha} \circ U_{p}=p \mathrm{Ha} .
$$

3. Lorsque $\epsilon<\frac{p-2}{p(2 p-2)}$ le morphisme induit $U_{p}: \phi_{\text {ord }}(\stackrel{\circ}{\epsilon}) \rightarrow \phi_{\text {ord }}\left(p^{\circ} \epsilon\right)$ est étale fini et surjectif. 
4. On a les relations de compatibilité suivantes

$$
\begin{aligned}
\pi_{1, n, k} \circ s_{n} & =s_{k \mid \oint_{\text {ord }}\left(\epsilon_{n}^{\circ}\right)} \\
s_{k} \circ \pi_{2, n-k, 0} \circ\left(s_{n-k}\right)_{\mid \oint_{\text {ord }}\left(\dot{\epsilon}_{n}\right)} & =\pi_{2, n, k} \circ s_{n} .
\end{aligned}
$$

Démonstration. - Le point (1) résulte du théorème 7. Le point (2) est une conséquence $\mathrm{du}$ théorème 5 . Le point (4) est contenu dans le théorème 6 . Pour le point (3), il est clair que le morphisme $U_{p}: \phi_{\text {ord }}(\stackrel{\circ}{\epsilon}) \rightarrow \phi_{\text {ord }}(p \epsilon)$ est étale. D'après la proposition 16 , il est surjectif. Il reste donc à voir qu'il est fini. Notons $V=\pi_{2,1,0}^{-1}\left(\phi_{\text {ord }}(p \epsilon)\right)$ un ouvert admissible de $\phi_{\mathfrak{P}_{1}}$. Soit $D \subset H[p]_{V}^{\text {rig }}$ le sous-groupe étale fini sur $V$ donné par la structure de niveau. Puisque $p \epsilon<\frac{1}{2}$, il y a un sous-groupe canonique d'échelon 1 sur $H_{V}^{\text {rig }} / D$. Soit donc $D \subset C \subset H\left[p^{2}\right]_{V}^{\text {rig }}$ où $C$ est le groupe étale fini tel que $C / D$ soit le sous-groupe canonique. Soit $U \subset V$ l'ouvert/fermé de $V$ où $C=D[p]$ et $p D=C$ (par ouvert/fermé on entend que l'on a un recouvrement admissible de la forme $\left.V=U \amalg U^{\prime}\right)$. L'image de $s_{1}: \phi_{\text {ord }}(\stackrel{\circ}{\epsilon}) \rightarrow \phi_{\mathfrak{P}_{1}}$ est contenue dans $V$ car le sous-groupe canonique d'échelon $2 \operatorname{sur} \phi_{\text {ord }}(\stackrel{\circ}{\epsilon})$ est localement libre sur $\mathbb{Z} / p^{2} \mathbb{Z}$.

\section{Perspectives}

\subsection{Optimalité}

Soit $H$ un groupe formel $p$-divisible de dimension 1 et de hauteur 2 sur $\theta_{K}$ (le groupe formel associé à une courbe elliptique à réduction supersingulière). On vérifie facilement les assertions suivantes :

$$
\begin{aligned}
\left(H[p]_{\mathrm{AS}}^{\lambda}\right)_{\lambda>0} \text { admet un cran de hauteur } 1 & \Longleftrightarrow \mathrm{Ha}(H)>\frac{p}{p+1} \\
\left(H[p]_{\mathrm{HN}}^{\lambda}\right)_{\lambda \in[0,1]} \text { admet un cran de hauteur } 1 & \Longleftrightarrow \mathrm{Ha}(H)>\frac{1}{2} .
\end{aligned}
$$

D'après la section 6 de [16], lorsque $\mathrm{Ha}(H)>\frac{1}{2}$ les crans de la filtration de HarderNarasimhan et d'Abbes-Saito de $H[p]$ coïncident. De ces calculs on déduit que le théorème 6 est optimal pour la filtration de Harder-Narasimhan mais que le théorème 4 ne l'est pas pour la filtration d'Abbes-Saito. Cependant, on va voir que cela n'est pas si important.

Supposons que $\mathrm{Ha}(H)>\frac{p}{p+1}$. Soit $C$ le cran de hauteur 1 de la filtration d'Abbes-Saito et $H^{\prime}=H / C$, alors

$$
\left\{\begin{array}{l}
\mathrm{Ha}\left(H^{\prime}\right)=p \mathrm{Ha}(H) \quad \text { si } \mathrm{Ha}(H)>\frac{1}{p+1} \\
\mathrm{Ha}\left(H^{\prime}\right) \in\left[\frac{p}{p+1}, 1\right] \quad \text { si } \mathrm{Ha}(H)=\frac{1}{p+1} \\
\mathrm{Ha}\left(H^{\prime}\right)=1-\mathrm{Ha}(H) \text { si } \frac{1}{p+1}>\mathrm{Ha}(H)>\frac{p}{p+1} .
\end{array}\right.
$$

En particulier, la borne du théorème 5 est optimale. De plus, on déduit de cela que lorsque $\frac{1}{2} \geq \mathrm{Ha}(H)>\frac{p}{p+1}$ alors $\mathrm{Ha}\left(H^{\prime}\right) \geq \mathrm{Ha}(H)$ et donc, dans la zone où il y a un sous-groupe canonique au sens d'Abbes-Saito mais pas au sens de la filtration d'Harder-Narasimhan, l'isogénie canonique fait grandir l'invariant de Hasse, ce qui n'était pas le but du jeu.

4 e SÉRIE - TOME $44-2011$ - No 6 


\subsection{Un meilleur invariant que celui de Hasse?}

Replaçons-nous dans le cadre de la section 8.2 et considérons pour simplifier uniquement le cas des variétés de Siegel. La variété de Siegel analytique rigide en niveau parahorique $\phi_{\mathfrak{P}_{1}}$ admet un modèle entier modulaire qui est le suivant. On considère les couples $(A, C)$, où $A$ est une variété abélienne principalement polarisée (munie d'une structure de niveau hors $p$ que l'on oublie dans les notations) et $C \subset A[p]$ est un sous-groupe fini localement libre de hauteur $g$ isotrope vis-à-vis de la polarisation. Notons $S_{\mathfrak{P}_{1}}$ le $\operatorname{Spec}\left(\mathbb{Z}_{p}\right)$-schéma représentant cet espace de modules. On a alors, $\phi_{\mathfrak{P}_{1}}=\widehat{S}_{\mathfrak{P}_{1}}^{\text {rig }}$. Il y a de plus une correspondance de Hecke

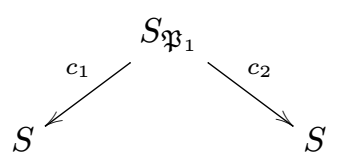

où $S$ désigne la variété de Siegel sans niveau en $p$. Par définition, $c_{1}$ envoie le couple $(A, C)$ sur $A$ et $c_{2}$ l'envoie sur $A / C$. Avec les notations de la section 8.2 on a donc $c_{1}^{\text {rig }}=\pi_{1,1,0}$ et $c_{2}^{\text {rig }}=\pi_{2,1,0}$. Si $A$ désigne la variété abélienne universelle sur $S$ posons $\mathscr{L}:=\operatorname{det}\left(\omega_{A}\right)$, un fibré automorphe sur les variétés de Siegel. L'invariant de Hasse est alors donné par la valuation d'une section de $\mathscr{L}^{\otimes(p-1)}$ modulo $p$. Sur $S_{\mathfrak{P}_{1}}$, si $f: A \longrightarrow A / C$ désigne l'isogénie universelle celle-ci fournit un morphisme $f^{*}: \omega_{A / C} \longrightarrow \omega_{A}$ qui est un isomorphisme en fibre générique. Passant au déterminant, cela nous donne une section

$$
\delta_{C} \in \Gamma\left(S_{\mathfrak{P}_{1}}, c_{1}^{*} \mathscr{L} \otimes c_{2}^{*} \mathscr{L}^{-1}\right)
$$

qui définit un diviseur de Cartier sur $S_{\mathfrak{P}_{1}}$ (il s'agit du diviseur associé à $C$ au sens de la section 2 de [16]). La valuation de ce diviseur définit une fonction

$$
\delta: \phi_{\mathfrak{P}_{1}} \longrightarrow[0, g] .
$$

Le point (1) du théorème 4 se retraduit sous la forme de la proposition suivante.

Proposition 17. - Soit $s_{1}: \phi_{\text {ord }}\left(\frac{i}{2}\right) \rightarrow \phi_{\mathfrak{P}_{1}}$ la section canonique. On a alors une égalité de fonctions

$$
\mathrm{Ha}_{\mid \delta_{\text {ord }}\left(\frac{i}{2}\right)}=g-\delta \circ s_{1} \text {. }
$$

Il semble à l'auteur de cet article que la fonction $\delta$ est plus facilement manipulable que l'invariant de Hasse.

\section{RÉFÉRENCES}

[1] A. Abbes, A. Mokrane, Sous-groupes canoniques et cycles évanescents $p$-adiques pour les variétés abéliennes, Publ. Math. Inst. Hautes Études Sci. 99 (2004), 117-162.

[2] A. Abbes, T. Saito, Ramification of local fields with imperfect residue fields, Amer. $J$. Math. 124 (2002), 879-920.

[3] F. Andreatta, C. Gasbarri, The canonical subgroup for families of Abelian varieties, Compos. Math. 143 (2007), 566-602.

[4] V. G. Berkovich, Vanishing cycles for formal schemes. II, Invent. Math. 125 (1996), 367-390. 
[5] P. Berthelot, Cohomologie cristalline des schémas de caractéristique $p>0$, Lecture Notes in Math. 407, Springer, 1974.

[6] P. Berthelot, Théorie de Dieudonné sur un anneau de valuation parfait, Ann. Sci. École Norm. Sup. 13 (1980), 225-268.

[7] P. Berthelot, L. Breen, W. Messing, Théorie de Dieudonné cristalline. II, Lecture Notes in Math. 930, Springer, 1982.

[8] S. Bosch, W. Lüt кевонmert, Formal and rigid geometry. I. Rigid spaces, Math. Ann. 295 (1993), 291-317.

[9] A. Chambert-Loir, Théorie de Dieudonné cristalline et périodes $p$-adiques, Bull. Soc. Math. France 126 (1998), 545-562.

[10] B. Conrad, Higher-level canonical subgroups in Abelian varieties, prépublication http://math.stanford.edu/ conrad/papers/subgppaper.pdf, 2006.

[11] P. Deligne, Travaux de Shimura, Séminaire Bourbaki 1970/71, exp. no 389, Lecture Notes in Math. 244 (1971), 123-165.

[12] R. Elkik, Solutions d'équations à coefficients dans un anneau hensélien, Ann. Sci. École Norm. Sup. 6 (1973), 553-603.

[13] G. Faltings, Integral crystalline cohomology over very ramified valuation rings, $J$. Amer. Math. Soc. 12 (1999), 117-144.

[14] L. Fargues, Application de Hodge-Tate duale d'un groupe de Lubin-Tate, immeuble de Bruhat-Tits du groupe linéaire et filtrations de ramification, Duke Math. J. 140 (2007), 499-590.

[15] L. Fargues, L'isomorphisme entre les tours de Lubin-Tate et de Drinfeld et applications cohomologiques, in L'isomorphisme entre les tours de Lubin-Tate et de Drinfeld, Progr. Math. 262, Birkhäuser, 2008, 1-325.

[16] L. FARGues, La filtration de Harder-Narasimhan des schémas en groupes finis et plats, J. reine angew. Math. 645 (2010), 1-39.

[17] J.-M. Fontaine, Groupes p-divisibles sur les corps locaux, Astérisque 47-48 (1977), 262.

[18] J.-M. Fontaine, Formes différentielles et modules de Tate des variétés abéliennes sur les corps locaux, Invent. Math. 65 (1981/82), 379-409.

[19] J.-M. Fontaine, Le corps des périodes p-adiques, Astérisque 223 (1994), 59-111.

[20] P. GABRIEL, Étude infinitésimale des schémas en groupe et groupes formels, in Schémas en Groupes (Sém. Géométrie Algébrique, Inst. Hautes Études Sci., 1963/64), Fasc. 2b, Exposé 7a, Inst. Hautes Études Sci., 1965, 1-65+4.

[21] E. Z. Goren, P. L. Kassaei, The canonical subgroup : a "subgroup-free" approach, Comment. Math. Helv. 81 (2006), 617-641.

[22] E. Z. Goren, P. L. Kassaei, Canonical subgroups over Hilbert modular varieties, C. R. Math. Acad. Sci. Paris 347 (2009), 985-990.

[23] B. H. Gross, M. J. Hopkins, Equivariant vector bundles on the Lubin-Tate moduli space, in Topology and representation theory (Evanston, IL, 1992), Contemp. Math. 158, Amer. Math. Soc., 1994, 23-88.

[24] L. ILlusie, Déformations de groupes de Barsotti-Tate (d'après A. Grothendieck), Astérisque 127 (1985), 151-198.

$4^{\mathrm{e}}$ SÉRIE - TOME $44-2011-\mathrm{N}^{\circ} 6$ 
[25] N. M. Katz, $p$-adic properties of modular schemes and modular forms, in Modular functions of one variable, III (Proc. Internat. Summer School, Univ. Antwerp, Antwerp, 1972), Lecture Notes in Math. 350, Springer, 1973, 69-190.

[26] M. Kisin, K. F. LAI, Overconvergent Hilbert modular forms, Amer. J. Math. 127 (2005), 735-783.

[27] F. F. Knudsen, D. Mumford, The projectivity of the moduli space of stable curves. I. Preliminaries on "det" and "Div", Math. Scand. 39 (1976), 19-55.

[28] R. E. Kоттwitz, Isocrystals with additional structure, Compositio Math. 56 (1985), 201-220.

[29] R. E. Kottwitz, Points on some Shimura varieties over finite fields, J. Amer. Math. Soc. 5 (1992), 373-444.

[30] J. Lubin, Canonical subgroups of formal groups, Trans. Amer. Math. Soc. 251 (1979), $103-127$.

[31] W. Messing, The crystals associated to Barsotti-Tate groups : with applications to Abelian schemes, Lecture Notes in Math. 264, Springer, 1972.

[32] J. Rabinoff, Higher-level canonical subgroups for $p$-divisible groups, prépublication arXiv:0910.3323.

[33] M. Raynaud, Schémas en groupes de type $(p, \ldots, p)$, Bull. Soc. Math. France 102 (1974), 241-280.

[34] J. Tate, p-divisible groups, in Proc. Conf. Local Fields (Driebergen, 1966), Springer, 1967, 158-183.

[35] J. Tate, F. Oont, Group schemes of prime order, Ann. Sci. École Norm. Sup. 3 (1970), $1-21$.

[36] Y. Tian, Canonical subgroups of Barsotti-Tate groups, Ann. of Math. 172 (2010), 955988.

[37] Y. Tian, lettre à Laurent Fargues.

[38] Y. Tian, An upper bound on the Abbes-Saito filtration for finite flat group schemes and applications, prépublication arXiv:1004.4205.

[39] T. Wedhor n, Ordinariness in good reductions of Shimura varieties of PEL-type, Ann. Sci. École Norm. Sup. 32 (1999), 575-618.

[40] T. Wedhorn, The dimension of Oort strata of Shimura varieties of PEL-type, in Moduli of Abelian varieties (Texel Island, 1999), Progr. Math. 195, Birkhäuser, 2001, 441-471.

[41] T. ZINK, The display of a formal p-divisible group, Astérisque 278 (2002), 127-248.

(Manuscrit reçu le 4 janvier 2010;

accepté, après révision, le 18 mars 2011.)

\author{
Laurent FARGUES \\ CNRS et Université Paris-Sud \\ Laboratoire de mathématiques d'Orsay \\ Bâtiment 425 \\ 91405 Orsay Cedex, France \\ E-mail: laurent.fargues@math .u-psud.fr
}

